\title{
Hyboptera Chaudoir, I872 of the Cryptobatida group of subtribe Agrina: A taxonomic revision with notes on their ways of life (Insecta, Coleoptera, Carabidae, Lebiini)
}

\author{
Terry L. Erwin', Shasta C. Henry² \\ I Hyper-diversity Group, Department of Entomology, MRC-187, National Museum of Natural History, \\ Smithsonian Institution, P.O. Box 37012, Washington D.C. 20013-7012, U.S.A. 2 School of Land and Food, \\ Faculty of Science, Engineering and Technology, University of Tasmania, Private Bag 78, Hobart, Tasmania, \\ Australia, 7001 \\ Corresponding author: Terry L. Erwin (erwint@si.edu) \\ Academic editor: L.Penev | Received 18 July 2017 | Accepted 17 October 2017 | Published 7 November 2017 \\ http://zoobank.org/BC4DF927-F547-446C-ACD3-F2B065E0842E \\ Citation: Erwin TL, Henry SC (2017) Hyboptera Chaudoir, 1872 of the Cryptobatida group of subtribe Agrina: A \\ taxonomic revision with notes on their ways of life (Insecta, Coleoptera, Carabidae, Lebiini). ZooKeys 714: 61-127. \\ https://doi.org/10.3897/zookeys.714.15113
}

\begin{abstract}
Hyboptera Chaudoir, 1872 of the Cryptobatida group of subtribe Agrina, Lebiini, living in the Neotropics and southernmost Nearctic realms are diagnosed, described, and illustrated, and new species are assigned to two inclusive species groups. Occurrences of species range from Texas, USA, to the state of Santa Caterina in Brazil. Seven new species of Hyboptera are described:

Hyboptera biolat Erwin \& Henry, sp. n.; Type locality - Perú, Madre de Dios, Manu Reserved Zone, Río Manu, BIOLAT Biological Station, Pakitza; Hyboptera vestiverdis Henry \& Erwin, sp. n.; Type locality - Perú, Madre de Dios, Manu Reserved Zone, Río Manu, BIOLAT Biological Station, Pakitza; Hyboptera lucida Henry \& Erwin, sp. n.; Type locality - French Guiana, Cayenne, Commune de Roura, Montagne des Chevaux; Hyboptera scheelea Erwin \& Henry, sp. n.; Type locality - Perú, Loreto, PacayaSamiria National Reserve, Río Samiria (South Branch), Camp Terry; Hyboptera shasta Erwin, sp. n.; Type locality - Brazil, Amazonas, north of Manaus on Amazonas 010 at Km 26, Reserva Ducke;

Hyboptera tepui Erwin \& Henry, sp. n.; Type locality - Venezuela, Amazonas, Cerro de la Neblina, Río Baria Basecamp; Hyboptera tiputini Erwin \& Henry, sp. n.; Type locality - Ecuador, Orellana, Yasuni National Park (edge), $95.43 \mathrm{~km}$ E (heading $101.46^{\circ}$ ) Coca, Tiputini Biodiversity Station. A revised identification key is provided to the genera of the Cryptobatida group and another to the species of Hyboptera
\end{abstract}

Copyright Terry L. Erwin, Shasta C. Henry. This is an open access article distributed under the terms of the Creative Commons Attribution License (CC BY 4.0), which permits unrestricted use, distribution, and reproduction in any medium, provided the original author and source are credited. 
Chaudoir and distributional data are provided for all known species of the latter. Adults of these species often occur in the canopy of many tropical tree species and records are reported where known. In addition, adults are found under the webbing of Psocoptera and in fleshy anther rings of Bombacaceae (Pseudobombax septenatum (Jacq.) Dugand), on the rain forest floor in the dry season. Larval and pupal stages of one species from Panamá are known from under bark of living fence posts; however, these immature stages are not treated in the current paper.

\section{Resumen}

Hyboptera Chaudoir, 1872, del grupo Cryptobatida, perteneciente a la subtribu Agriina, Lebiini, viviendo en los Neotrópicos y en las áreas meridionales del Neártico se diagnostican, describen e ilustran y nuevas especies se asignan a dos grupo-especies inclusivos. La distribución de las especies recorre desde Texas, EE.UU., hasta el estado de Santa Caterina en Brasil. Siete nuevas especies de Hyboptera se describen:

Hyboptera biolat Erwin \& Henry, sp. n.; Type locality - Perú, Madre de Dios, Manu Reserved Zone, Río Manu, BIOLAT Biological Station, Pakitza; Hyboptera vestiverdis Henry \& Erwin, sp. n.; Type locality - Perú, Madre de Dios, Manu Reserved Zone, Río Manu, BIOLAT Biological Station, Pakitza; Hyboptera lucida Henry \& Erwin, sp. n.; Type locality - French Guiana, Cayenne, Commune de Roura, Montagne des Chevaux; Hyboptera scheelea Erwin \& Henry, sp. n.; Type locality - Perú, Loreto, PacayaSamiria National Reserve, Río Samiria (South Branch), Camp Terry; Hyboptera shasta Erwin, sp. n.; Type locality - Brazil, Amazonas, north of Manaus on Amazonas 010 at Km 26, Reserva Ducke;

Hyboptera tepui Erwin \& Henry, sp. n.; Type locality - Venezuela, Amazonas, Cerro de la Neblina, Río Baria Basecamp; Hyboptera tiputini Erwin \& Henry, sp. n.; Type locality - Ecuador, Orellana, Yasuni National Park (edge), $95.43 \mathrm{~km}$ E (heading 101.46 ) Coca, Tiputini Biodiversity Station. Se provee una clave revisada para los géneros dentro del grupo Cryptobatida y otra para las especies del género Hyboptera Chaudoir y se incluye información sobre la distribución de todas las especies conocidas de éste último. Los adultos de estos comúnmente se encuentran en el dosel de muchas especies de árboles tropicales y registros se reportan cuando se conocen. Además, los adultos pueden estar debajo de las telas de sócidos y en los anteridios carnosos de los Bombacaceae (Pseudobombax septenatum (Jacq.) Dugand) en el suelo del bosque lluvioso. Las etapas de larva y pupa de una de las especies de Panamá se conocen viviendo debajo de la corteza de postes vivos de cercas; sin embargo, estos so se tratan en este artículo.

\section{Keywords}

Neotropics, Nearctic, Texas, Embioptera, Psocoptera, rain forest, Hyboptera Chaudoir, Thoasia Liebke, Straneotia Mateu

\section{Palabras claves}

Neotrópicos, Neártico, Texas, Embioptera, Psocoptera, bosque lluvioso, Hyboptera Chaudoir, Thoasia Liebke, Straneotia Mateu

\section{Introduction}

The Cryptobatida Group of Lebiini was proposed to include a number of genera including Hyboptera Chaudoir (Erwin 2004). This genus was treated most recently by Reichardt $(1971,1973)$ and those reports were brief and superficial, not full revisions. Before that, only five isolated species descriptions by Chaudoir, Reiche and Oberthür existed. Hence, this small group of very attractive and biologically interesting beetles requires an initial taxonomic revision. The purpose of this paper is to rapidly validate some new 
species names (Erwin and Johnson 2000) with descriptions and provide more complete re-descriptions of known species, so that the group is available for further study, particularly their way of life in association with Psocoptera. Hyboptera are members of the Cryptobatida group by virtue of the structure of their mouthparts, elytral transverse depression, and the male genitalia with a flagellum. Herein, we provide full descriptions of all known taxa, including color images, an up-to-date map of their known distributions, and what is known of their ways of life including host tree species.

\section{Methods and materials}

(modified from Erwin and Zamorano 2014)

As noted in several past contributions, methods and species concepts follow those previously described (Erwin and Kavanaugh 1981; Kavanaugh and Erwin 1991). The species validation and diagnosis format follows as closely as possible that suggested in Erwin and Johnson (2000). For measurements, images of the specimens were taken using a Leica M420 stereoscope coupled to an EntoVision ${ }^{\mathrm{TM}}$ system. The resulting image was processed using the software Cartograph version 7.2.5 by Microvision Instruments. The magnification on the zoom was set to calibrate the system and it is embedded into the file of the image. The image was then opened with the software program Archimed version 6.1.4, also by Microvision, and the Measure tool was then used to determine the lengths of the various parts. A total of 239 images were obtained. Measurements of length (ABL, SBL) and width (TW) follow those of Ball (1972) and Kavanaugh (1979): ABL (apparent body length), measured from apex of labrum to apex of longer elytron (in adults of this genus, the abdomen often protrudes beyond the elytral apex, thus the ABL often is much larger that the SBL; SBL (standardized body length), equals the sum of the lengths of the head (measured from apex of clypeus to a point on midline at level of the posterior edge of compound eyes); PL (pronotum length) is measured from apical to basal margin along midline; LE (elytron length) is measured from apex of scutellum to apex of the longer elytron; TW (total width) is measured across both elytra at their widest point with suture closed; and WH (head width) is measured from extreme margin of protuberant eyes left to right. Note that not all specimens available were measured because more than 33 specimens were available, thus we limited "n" to 33 as a statistically valid sample size. Sexes were measured separately, we found slight differences among the species sexes, and hence we report measurements for both sexes in our Tables (see Appendix 1). For the Hyboptera treatment below, we provide relative size terms based on the SBL as follows: small-size $<3.3$ $\mathrm{mm}$, medium-size $3.3 \mathrm{~mm}$ to $4.3 \mathrm{~mm}$, and large-size $>4.3 \mathrm{~mm}$. For an explanation of the measurements and their incorporation in Appendix 1, see Erwin (2011) and Erwin and Ball (2011). For the present study, we report the harmonic mean, as we believe it better reflects the central tendency than the arithmetic mean.

Attributes of the abdominal ventral sterna are referred to using the numbering system generally accepted in carabid studies, i.e., the sternum divided medially by the hind 
coxae is sternum II (the first being hidden) and the last visible is sternum VII (Liu et al. 2011). In a revision of the genus Pericompsus (Erwin 1974), a problem was encountered with the term "stria" for features of their punctate elytra (i.e., the so-called striae were not actually striae, rather they were rows of punctures). The result was the use of the term "interneur" to apply to the attribute lying between intervals. Through use of this term, one could describe the feature as interneur striate, punctate, striatopunctate, etc.

A similar problem exists for the proximal end of the median lobe of the male genitalia. In Snodgrass (1935), the term "phallobase" is used, and we have adopted it here (see Erwin 2011). Therefore, by extension, in Carabidae, we can say phallobase hooded (e.g., Lebiini, Pseudomorphini), phallobase of two parallel sclerotized struts (basal trechines and Andinodontis), phallobase of two uneven struts (Bembidion), etc. Kavanaugh (pers. comm.) points out that with struts there are still connecting membranes surrounding the struts forming a "bulb." We have chosen the aedeagal illustration of a male $H$. angulicollis Chaudoir (Fig. 5A) and H. apollonia Erwin (Fig. 10A) to display the identifying code letters and these apply to all illustrations of male genitalia of Hyboptera included.

This study includes 738 adult specimens of Hyboptera, all currently at the National Museum of Natural History, Washington, DC (NMNH) and, where appropriate, returned to their corresponding institutions upon publication. Among these specimens, some were received from:

AMNH American Museum of Natural History, New York, NY, USA (Lee Herman)

BMNH Natural History Museum, London, UK (Beulah Garner)

CAS California Academy of Sciences, Berkeley, CA, USA (David H. Kavanaugh)

CMNH Carnegie Museum of Natural History, Pittsburgh, PA, USA (Robert L. Davidson)

HESP Henry Hespenheide Private Collection, Los Angeles, CA, USA (Henry Hespenheide)

IAvH Instituto de Investigación de Recursos Biológicos, Alexander von Humboldt, Bogotá, Colombia (Arturo González)

INBIO Instituto Nacional de Biodiversidad, Santo Domingo, Costa Rica (Angel Solis)

LACM Los Angeles County Museum, Los Angeles, CA, USA (Brian V. Brown)

MCZ Museum of Comparative Zoology, Harvard University, Boston, MA, USA (Philip Perkins)

MNHP Muséum national d'Histoire naturelle, Paris, France (Thierry Deuve)

NBCL Naturalis Bidiversity Center, Lieden, Netherlands (Luc Willemse)

OSU C.A. Triplehorn Insect Collection, Ohio State University, Columbus, OH, USA (Charles A.Triplehorn)

SEMC Snow Entomological Museum, University of Kansas, Lawrence, Kansas, USA (Zachary Falin)

UASM EH Strickland Entomological Museum, University of Alberta, Edmonton, Canada (George E. Ball \& Danny Shpeley)

UCD Bohart Museum of Entomology, University of California, Davis, CA, USA (Lynn Kimsey)

UNMSM Universidad Nacional Mayor de San Marcos, Lima, Perú (Gerardo Lamas) 
Primary type specimens of new species will be deposited in their countries of origin if required by legal agreements, or museums of ownership at the conclusion of our studies on this tribe.

The enhanced habitus images of the adult beetles portray most of the character states referred to in the key provided. Illustrations of male genitalia are standard for descriptive taxonomy of carabid beetles in both preparation and aspects presented, as is the presentation of the female genitalia (one example per genus, in this case $H$. lucida sp. n.). The habitus images of the adults were made with a Visionary Digital ${ }^{\mathrm{TM}}$ high resolution imaging system rendered using Photoshop to become "Digital Photoillustrations." Figure captions include an ADP number, which is a unique identification number for the specimen that was imaged and links the specimen and associated illustrations and/or images to additional information, such as collecting notes, in electronic databases at the NMNH.

Geographical data are presented for species based on all known specimens available at the time of manuscript preparation, including those in the literature. Georeferenced data have been determined from locality information provided on specimen labels; only those exact geo-references reported in decimal degrees that are provided on the label are placed in quotes. Otherwise, we have estimated others as closely as possible from places, mileage, or other locality data listed on the label and searched with Google Earth Pro. Latitude and longitude for those are reported in decimal degrees and have been corrected from those reported on the labels, if necessary; our bottom line is that georeferenced locality data reported herein are far more accurate than those provided on specimens labels.

A distribution map is provided for the species of Hyboptera (Fig. 11). Here, vernacular names in English are proposed, as common names are becoming increasingly needed in conservation reports and studies, and/or agricultural and forestry applications. These names are based on criteria set forth in Erwin (2011a) and applied in Erwin (2011b).

Host occurrences of rainforest trees are reported using the names provided by botanists who inventoried two fogging transects established by the senior author (TLE) in Ecuador. These names have not been elaborated with author names herein, as is traditional in botanical literature, however, they can be readily found on the internet.

\section{Accounts of taxa}

\section{Tribe Lebiini, Subtribe Agrina, Cryptobatida Group}

Diagnosis. Adults. Head ventrally without suborbital setigerous punctures, neck not markedly narrowed, except somewhat in Thoasia. Mandible widened near base, scrobe wide, lateral margin markedly rounded; dentition of occlusal margins reduced, typical for Lebiini (cf. Shpeley and Ball 2001: figs 6-9); palpi with ultimate articles subsecuriform or securiform, paraglossae broad, glabrous, adherent, extended to anterior angle of glossal sclerite. Elytron usually with transverse depression at anterior third, appearing deformed; penultimate setigerous puncture of elytron umbilicate series not 
displaced laterally nor medially. Posterior tibial spurs subequal, their margins smooth; tarsomere 4 usually bilobed. Except in one genus (i.e., some species of Aspasiola), endophallus with flagellum [the absence from males of some species of Aspasiola species probably represents a reversal (secondary loss)].

Notes. Subtribe Agrina consists of those species formerly included in the Subtribe Calleidina (cf. Lorenz 2005). The Cryptobatida Group, by virtue of the attributes above, has the type genus Cryptobatis Eschscholtz. Many records of species in most genera refer to collections on fungus arrayed on sides or the bottom of fallen tree trunks. It is probable that the Cryptobatida Group contains many species whose adults are predators on other shelf fungus inhabitants (cf. Erwin and Erwin 1976, wherein adults and larvae of a species of Eurycoleus, which is a member of the subtribe Pericalina, are predaceous on fungal feeders; other pericalines are also associated with fungi). Hyboptera and its adelphotaxon Hybopteroides are exceptions (Erwin and Ball 2012), as the adults are likely predators on psocids and embiids beneath silken webs, respectively.

Below, we have added to the Cryptobatida Group the genus Straneotia Mateu 1961 by virtue of a study of new specimens collected by fogging and flight intercept traps (FIT) in Ecuador and French Guiana, respectively. Mateu erected this genus based on a single female specimen from eastern Amazonian Brazil (Straneotia freyi Mateu) and described a second species in the genus based on a single female from western Amazonian Brazil (Straneotia amazonica Mateu). These two species soon will be redescribed and the new species described (Erwin and Aldebron, in prep).

\section{Key to the genera of the Cryptobatida Group, Subtribe Agrina (Lebiini)}

(Enhanced from Erwin 2004 and Erwin and Ball 2012)

1 Elytron at basal third depressed, surface uneven, tuberculate or not, and/or margin of pronotum angulate or sub-angulate at mid-lateral setiferous pore, or tubular Cryptobatida Group...2

1' Elytron neither depressed nor tuberculate, surface smooth with normal interneurs and intervals (or interneurs effaced); side of pronotum evenly rounded Plochionida Group, Calleidida Group, Agrida Group

2(1) Elytron markedly tuberculate overall, or with a series of small setiferous tubercles on intervals 3 and 5, lateral marginal intervals without callus..... 3

2' Elytron without trace of discal tubercles; lateral marginal intervals with or without callus

3(2) Prothorax somewhat tubular, much narrower than head

Otoglossa Chaudoir, 1872

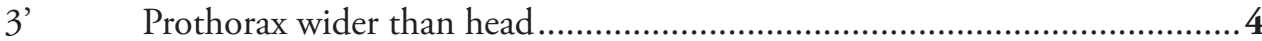

$4\left(3^{\prime}\right) \quad$ Sides of pronotum narrowly reflexed except at mid-lateral seta, there wide; neck well-defined, narrow; elytra metallic blue, fore-body all or mostly rufous.

Thoasia Liebke, 1939

4 Sides of pronotum broad and margins broadly reflexed throughout; neck broad; color otherwise. 
5(4') Elytron with numerous tubercles on at least three intervals; head dorsum transversely rugose; side margins of pronotum subtly angulate or not; labrum large, broadly bilobed; tarsomere 4 bilobed...... Hyboptera Chaudoir, 1872

5' Elytron not tuberculate; head dorsum longitudinally strigose (wrinkled); side margins of pronotum markedly angulate; labrum normal, rectangulate, slightly emarginate or truncate apically; tarsomere 4 not bilobed

Hybopteroides Erwin \& Ball, 2012

6(2') Antennomere 4 glabrous except for apical ring setae ..............................7

6) Antennomere 4 multisetiferous from basal third to apex, in addition to apical

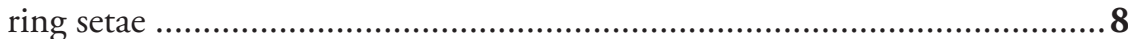

7(6) Elytron laterally with callus at apical third; male endophallus without flagellum Valeriaaschero Erwin, 2004

7’ Elytron laterally without callus at apical third; male endophallus with or without flagellum

Aspasiola Chaudoir, 1877

8 (6') Head markedly narrow, elongate, and tubular; eyes more or less flat

Straneotia Mateu, 1961

8' Head normal with large hemispheric eyes .......................................9

9(8') Head and pronotum densely and evenly punctate.

Cylindronotum Putzeys, 1845

9' Head and pronotum smooth ......................................................... 10

10 (9') Pronotum with lateral margin narrowly reflexed from base to apex

Pseudotoglossa Mateu, 1961

10' Pronotum with lateral margin moderately or markedly reflexed from base to

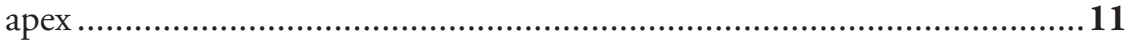

$11(10$ ') Elytron laterally at apical third with large callus

Cryptobatis Eschscholtz, 1829

11' Elytron laterally at apical third without callus Onota Chaudoir, 1872

\section{Hyboptera Chaudoir, 1872}

HuMPS-BACKED BEETLES

Figs 1-11

Hyboptera Chaudoir, 1872: 161. Type species: Hyboptera angulicollis Chaudoir, 1872: 164, designated by Reichardt (1973:50).

Aspasia Reiche, 1842: 310 (not Dejean 1831). Type species: Aspasia verrucosa Reiche, 1842: 310. Synonymized by Chaudoir 1872: 161.

Diagnosis. (cf. Figs 1-10). Neck broad; eyes very large, hemispherical; frons markedly rugose, more or less anteriorly depressed. Prothorax wider than head; sides of pronotum broadly reflexed throughout, rounded or subangulate at mid-lateral setigerous pore. Elytron at basal third very slightly transversely depressed, surface uneven, markedly tuberculate overall. Flight wings of a dusky color. Basitarsus of mid and hind legs markedly elongate, coequal to length of tarsomeres 2-5 combined; males with adhesive 
setae on tarsomeres 1-3. Endophallus with flagellum; flagellum extruded at apex in many specimens.

Dispersal potential. The wings are fully developed in adults of all known species, thus it is likely these beetles are moderate to strong flyers.

Geographic distribution. A widespread southern Nearctic and Neotropical genus known from Texas, USA, south to southeastern Brazil, in the west to Bolivia, and east to French Guiana.

Ways of life. Much is known about the species in this genus and that is reported here for the first time. Adults of various species are regularly collected in both the wet and dry seasons using insecticidal fogging techniques in many species of trees reaching the forest canopy in the Amazon Basin, thus they are certainly mainly arboreal. They are good flyers as evidenced by their capture in Malaise traps, C.D.C. mosquito traps, FITs, and at different types of light traps. At Barro Colorado Island in Panamá, one of us (TLE) collected adults from the forest floor amongst the large shed anther rings of trees of the species Pseudobombax septenatum (Jacq.) Dugand; these rings being a moisture source on the forest floor during the dry season (Erwin 1991, 2004). Warren E. Steiner collected several larvae and pupae along with emerging adults of Hyboptera verrucosa (Reiche) under bark of a living fence post in Panamá. These immature stages have yet to be described and illustrated (Erwin and Steiner, in prep).

Notes. Five species have been previously described in this genus, along with three species in its adelphotaxon, Hybopteroides Erwin \& Ball, 2012. Apparently, Lorenz (1998) was unaware of the Reichardt (1973) paper designating a type species (H. angulicollis Chaudoir) for the genus and unnecessarily designated $H$. tuberculata (Dejean), as the type species. The former stands.

Included species. The species list below, as well as the arrangement of descriptions that follows, is ordered alphabetically within two species groups.

\section{angulicollis species group}

Hyboptera angulicollis Chaudoir, 1872:164; Brazil - Pará; Colombia; Ecuador; French Guiana; Perú; Suriname.

Hyboptera biolat Erwin \& Henry, sp. n.; Perú.

Hyboptera vestiverdis Henry \& Erwin, sp. n.; Ecuador; Perú.

Hyboptera scheelea Erwin \& Henry, sp. n.; Perú.

Hyboptera shasta Erwin, sp. n.; Brazil - Amazonas.

Hyboptera tepui Erwin \& Henry, sp. n.; Venezuela.

Hyboptera tiputini Erwin \& Henry, sp. n.; Colombia; Ecuador; Perú.

Hyboptera viridivittis Chaudoir, 1872:164; Brazil - Minas Gerais, Rio de Janeiro, Santa Catarina. 


\section{tuberculata species group}

Hyboptera apollonia Erwin, 2004: 33; Costa Rica; Panamá.

Hyboptera auxilidora Erwin, 2004: 35; Costa Rica; Honduras; México - VC; Panamá, USA - TX.

Hyboptera dilutior Oberthür, 1884: 52; Brazil - Amazonas, Pará, Rondônia; Ecuador;

French Guiana; Perú; Venezuela.

Hyboptera lucida Henry \& Erwin, sp. n.; Ecuador; French Guiana.

Hyboptera tuberculata (Dejean), 1825: 272; Bolivia; Brazil - Amazonas, Sergipe; Colombia; Ecuador; Guyana; French Guiana; Perú; Suriname.

Hyboptera verrucosa (Reiche), 1842: 311; Brazil - Amazonas; Colombia; Ecuador;

French Guiana; Panamá; Perú; Suriname; Trinidad and Tobago; Venezuela.

\section{Accounts of taxa}

\section{Key to the species of Hyboptera Chaudoir, 1872}

$1 \quad$ Adults with only dark non-metallic markings on the pronotal disc ............2

1' Adults with patches of bright metallic green para-medially on pronotum....7

1" Adults without or with only slightly darker, non-metallic pronotal marking ... 11

2(1) Pronotum with discal rugae etched horizontally and linear (Fig. 6A)

H. apollonia Erwin

2' Pronotum with discal rugae etched at an angle aimed medio-posteriorly, or somewhat chaotically without clear order................................................ 3

3(2') Elytra black with 4 small medio-apical tubercles pale (Fig. 7B) .................... H. lucida Henry \& Erwin, sp. n.

3' Elytra mostly testaceous or pale brownish with darkly marked tubercles; small medio-apical tubercles pale or not...................................................4

4(3') Elytron with sutural margin at apical sixth black markedly contrasting with testaceous background color (Fig. 7A)......................H. dilutior Oberthür

4 Elytron with sutural margin at apical sixth pale, not black, if brownish not contrasting with background color.

5(4') Elytron just posterior to scutellum with only the suture pale in color otherwise markedly infuscate (Fig. 9A)........................H. tuberculata (Dejean)

5' Elytron just posterior to scutellum with a V-shaped pale area encompassing the suture and the first interval

6(5') Elytron narrow, almost parallel sided. Distribution from Panamá south into the Amazon Basin and across to French Guiana and Trinidad \& Tobago (Fig. 9B)

6' Elytron broad with markedly arcuate lateral margin. Distribution from Panamá north to southernmost Texas (Fig. 6B) H. auxilidora Erwin 
7(1') Elytra entirely metallic bronzy-green ........................................................ 8

7' Elytra dark matte black or dark and markedly shiny, some individuals with hint of metallic green spots near basal margin ...........................................9

8(7) Venter with meso- and metathorax mostly infuscated (Fig. 1B)

8' Venter entirely rufotestaceous (Fig. 1A) H. biolat Erwin \& Henry, sp. n.

9(7') Elytra dark matte black (Fig. 4B) H. angulicollis Chaudoir

9' Elytra markedly shiny H. viridivittis Chaudoir

10(9') Elytron violaceous, broad; meso- and metathorax pale not contrasting with pale abdomen (Fig. 4A) H. tiputini Erwin \& Henry, sp. n.

10' Elytron dark olivaceous, narrow; meso- and metathorax infuscated, markedly contrasting with pale abdomen (Fig. 2A) H. vestiverdis Henry \& Erwin, sp. $\mathrm{n}$.

11(1") Elytron with lateral margin broadly testaceous from humerus to sutural apex; apical abdominal tergite testaceous with slight infuscation at extreme posterior-lateral corners (Fig. 3B) H. tepui Erwin \& Henry, sp. n.

11'. Elytron with lateral margin narrowly testaceous to latero-apical corner, not reaching sutural apex; apical abdominal tergite mostly infuscated with narrow median testaceous stripe. 12

12(11') Elytra brilliant metallic green throughout, size larger (Fig. 3A) H. shasta Erwin, sp. n.

12'. Elytra blackish-blue with metallic green highlights across the humeri and green points at some larger tubercles (Fig. 2B)

H. scheelea Erwin \& Henry, sp. n.

\section{angulicollis species group}

(recognized by Reichardt 1973)

The most distinctive attribute of species in this group is that the pronotum has marked discal rugae etched almost horizontally and linear, as opposed to angulate. Adults of all have metallic green coloration somewhere on the dorsal surface and the general adult size is small to medium for the genus. Male phallus apex elongate, somewhat acuminate.

\section{Hyboptera angulicollis Chaudoir, 1872}

Angled-neck humps-backed beetle

Figs $1 \mathrm{~A}, 5 \mathrm{~A}, 11$

Hyboptera angulicollis Chaudoir, 1872: 164.

Holotype. Sex unknown. Brazil, Amazonas, Ega (Tefé), (HW Bates)(MNHP). Not seen by us; however, we have seen a "homotype" labelled by George E. Ball who studied the holotype in Paris. 
Derivation of specific epithet. The epithet, angulicollis, is a Latinized singular feminine adjective meaning "angled neck" referring to the angulate lateral sides of the pronotum.

Proposed english vernacular name. Angled-neck humps-backed beetle.

Diagnosis. With the attributes of the genus and angulicollis species group as described above and adults with patches of bright metallic green para-medially on pronotum, elytra entirely with metallic green reflection, and venter entirely rufotestaceous.

Description. (Figs 1A, 5A). Size: See Appendix 1. Length (SBL) long for genus, $\mathrm{ABL}=3.943-4.800 \mathrm{~mm}, \mathrm{SBL}=3.34-4.15 \mathrm{~mm}, \mathrm{TW}=1.92-2.53 \mathrm{~mm}$.

Color: See diagnosis, above.

Luster: Metallic highlights, partially iridescent.

Microsculpture: Mostly isodiametric or slightly stretched, well-impressed, cells somewhat more stretched around elytral tubercles.

Head: Rugae moderately coarse, mostly transverse. Eye very large, sub-hemispheric, and evenly rounded anteriorly, subtly more prolonged posteriorly. Antenna short, barely reaching humerus. Labrum rectangulate, shallowly bilobed, anterior margin slightly emarginate. Neck finely transversely rugose.

Prothorax: Pronotum moderately broad, disc centrally planar with dense transverse rugae. Lateral margins broadly explanate and obtusely angled medially then subtly arcuate to broadly obtuse hind angle, base medially produced and rounded posteriorly.

Pterothorax: Normal for Agrina, fully winged. Elytron intervals 3 with 6, and 5 with 5 (4) discal unisetiferous tubercles, other intervals moderately convex, side margin moderately explanate laterally only at middle third. Elytron broad and moderately short, moderately narrower than the pronotum at the broadest part, apex truncate, slightly rounded with distal corner broadly and obtusely rounded, disc not significantly convex, basal third slightly depressed. All interneurs well-impressed.

Legs: Femur dorso-ventrally moderately depressed, tibia coequal in length, more depressed; tarsus less than half the length of the tibia, fourth tarsomere markedly bilobed and with tarsal pad of setae.

Abdomen: Sparsely setiferous; normal ambulatory setae on sterna 3-5; female with two pairs of ambulatory setae on sternum 6 , medial pair of setae less than the length of lateral pair; males with only the lateral pair of longer setae on sternum 6.

Male genitalia: Phallus (Fig. 5A) with ostium of 1/5 its length, catopic, apex long, narrowly rounded; endophallus with flagellum (not obvious in illustration), flagellum not barbed. Parameres asymmetric, right very small, left larger.

Female genitalia: Not investigated, likely similar to that of $H$. lucida (Fig. 8).

Variation: In the large series of specimens of this species available to us for this study from many localities, we noted that the pronotal explanate margins vary considerably in width, the more narrow they are the more prominent the midpoint angle, the broader the explanation the more obtuse the midpoint angle.

Dispersal potential. These beetles are macropterous and capable of flight. They are moderately swift and agile runners. Specimens have been acquired using most modern collecting methods, including insecticidal foggings, UV light sheets and traps, Malaise traps, SLAM Malaise traps, and flight intercept traps (FITs). 

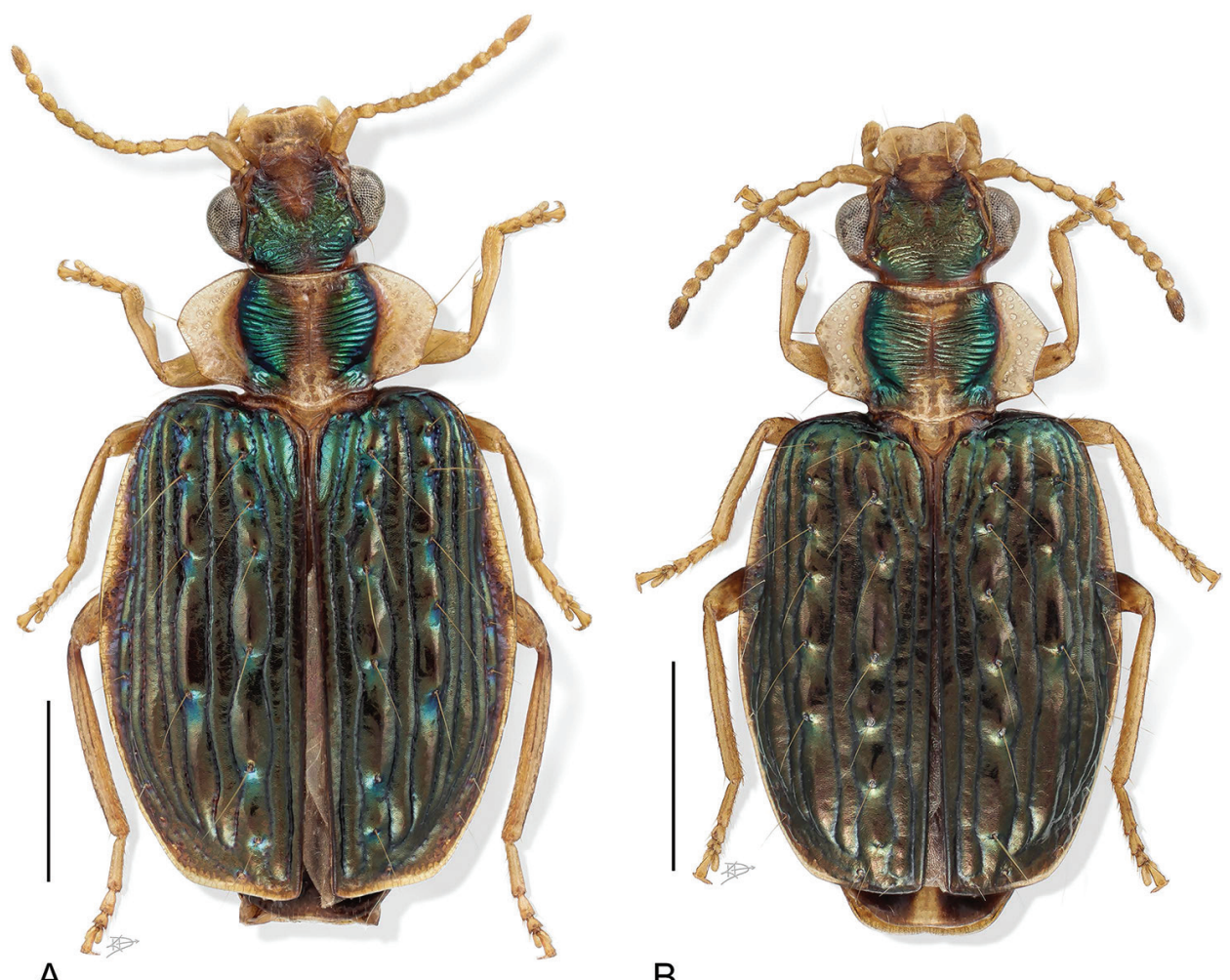

Figure I. Digital Photo-illustration. Habitus, dorsal aspect. A Hyboptera angulicollis Chaudoir, female, ADP052729 B Hyboptera biolat Erwin \& Henry, sp. n., female, ADP007398.

Way of life. Adults are common in the lowlands (8 to $700 \mathrm{~m}$ a.s.l.) and appear to be generalists in a variety of rainforest biotopes including terra firme, várzea, igapó, second growth, subtropical moist and white sand forests. In these forests, they are commonly found in big trees with vines and epiphytes, in suspended dry leaves, under lianas close to the trunk, in dry Scheelea sp. and Astrocaryum chambira Burret palm fronds, in very large suspended dry Cecropia leaves, and in dry bamboo leaves of the genera Guadua [G. weberbaueri Pilg.] and Elytrostachys [sp.] Individuals can be found in all months of the year, in both the rainy and dry seasons. Member of this species have been recorded from the canopy of the following tree species using insecticidal fogging techniques: Allophyllus sp.; Pourouma bicolor ssp. bicolor cf., Pourouma cecropiifolia aff., Pourouma mollis ssp. triloba cf.; Virola obovate, Virola decorticans cf.; Brosimum utile ssp. ovatifolium cf., Aspidosperma darienense cf.; Crepidospermum rhoifolium; Castilla ulei cf.; Pseudolmedia laevis, Pseudolmedia macrophylla cf.; Hymenaea oblongifolia; Matisia malacocalyx cf.; Oenocarpus bataua; Siparuna decipiens; Trattinnickia rhoifolia var. lancifolia cf.; Cecropia herthae, Cecropia ficifolia; Pentagonia spathicalyx cf.; Pouteria reticulata ssp. reticulata cf., Pouteria cuspidata ssp. robusta cf., Pouteria baehniana cf., Pouteria rostrata aff. ańangu; Gustavia longifolia; Leonia glycicarpa; Protium sagotianum 
cf., Protium nodulosum cf.; Sloanea cordia; Iriartea deltoidea; Browneopsis ucayalina; Eschweilera coriacea cf., Eschweilera juruensis cf.; Batocarpus orinocensis cf.; Pachira aquatica cf.; Sarcaulus brasiliensis aff. burnt; Blakea sp. 1; Parkia multijuga cf.; Swartzia león; Rhodostemonodaphne kunthiana cf.; Nectandra sp. 1, Nectandra crassiloba cf.; Inga olivaceae, Inga capitate, Inga bourgonii cf., Inga cuadra; Rinorea viridifolia; Talisia bitter; Coussapoa orthoneura cf., Coussapoa herthae; Aspidosperma darienense cf.; Vismia weedy; Astrocaryum chambira; Tabebuia moby; Wittmackanthus stanleyanus cf.; Alchornea triplinervia cf.; Maquira calophylla; Trichilia elsae cf., Trichilia solitudinis; Trichilia solitudinis; Simira cordifolial rubescens cf.; Catatola costaricana aff.; Brownea grandiceps cf.; Pausandra trianae; Micropholis venulosa cf.; Sagotia racemosa; Diospyros sericea; Guarea silvatica; Sorocea pubivena ssp. oligotricha cf.; Grias neuberthii; Simaba guianensis cf.; Tapura peruviana cf.; Neea dive-tuberculate; Semaphyllanthe megistocaula cf., Semaphyllanthe garcinia macrophylla cf.; Guatteria sp. 3, sect.; Meiocarpus, long petiole.

Other specimens examined. BraziL, Pará, Belém, Mocambo, $1.555^{\circ} \mathrm{S}$, 48.429 $\mathrm{W}, 25 \mathrm{~m}, 10$ January 1978 (NMNH: ADP007291, female), 20 May 1978 (NMNH: ADP007290, female), 23 June 1978 (NMNH: ADP007289, female). Colombia, Amazonas, PNN Amacayacu, February 1989 (M Kelsey)(IAvH: IAvHE-2434, ADP145214, IAvH-E-2435, ADP145200, males), IAvH-E-2436, ADP145206, IAvH-E-2437, ADP145202, IAvH-E-2438, ADP145204, IAvHE-73774, ADP145210, females), 100m, July 1988 (M Kelsey)(IAvH: IAvH-E-40649, ADP145215, female), 100m, 19 January 1998 (A Alvarado)(IAvH: IAvH-E-2439, ADP145217, female), 150-200m, 31 August 1997 (D Campos)(IAvH: IAvHE-73773, ADP145149, male), 1 September 1997 (D Campos, F Fernandez)(IAvH: IAvH-E-73773, ADP145147, male, IAvH-E-2442, ADP145191, female), Boca Caño Matamata, 3.3839 $\mathrm{S}, 70.0999^{\circ} \mathrm{W}, 150 \mathrm{~m}, 30$ August 1997 (Brown, G Kung)(IAvH: IAvH-E-2441, ADP145209m, female), Caño Matamata, $3^{\circ} 23^{\prime} \mathrm{S}, 070^{\circ} 06^{\prime} \mathrm{W}, 150 \mathrm{~m}$, 11-17 December 2000 (A Parente)(IAvH: IAvH-E-3534, ADP145195, male), San Martin, $3.776^{\circ} \mathrm{S}, 070.301^{\circ} \mathrm{W}, 80 \mathrm{~m}, 22-30$ April 2000 (B Amado)(IAvH: IAvHE-73757, ADP145189, female), 2-7 July 2000 (B Amado)(IAvH: IAvH-E-73763, ADP145211, female), 13-21 December 2000 (B Amado)(IAvH: IAvH-E-3533, ADP 145197, female), CAQuetá, Florencia, Santa Rosita, 0120' N, 076 $6^{\circ} \mathrm{W}, 600 \mathrm{~m}$, 7-22 July 2000 (E González)(IAvH: IAvH-E-2284, ADP145160, IAvH-E-40757, ADP145162, males), 22 July - 4 August 2000 (F Ruales)(IAvH: IAvH-E-73770, ADP 145164, female), PNN Chiribiquete, Puerto Abeja, $00^{\circ} 4^{\prime} \mathrm{N}, 072^{\circ} 26^{\prime} \mathrm{W}, 310 \mathrm{~m}$, 29 October - 12 November 2000, M Ospina, E González)(IAvH: IAvH-E-2699, ADP145167, IAvH-E-73765, ADP145156, IAvH-E-73766, ADP145158, females), PNN Chiribiquete, Puerto Solano, $00^{\circ} 12^{\prime} \mathrm{N}, 072^{\circ} 25^{\prime} \mathrm{W}, 300 \mathrm{~m}, 19-22$ February 2001 (M Ospina, E González)(IAvH: IAvH-E-73771, ADP145198, male), 26 February - 3 March 2001 (M Ospina, E González)(IAvH: IAvH-E-73772, ADP145192, female), 12-19 November 2000 (J Forero)(IAvH: IAvH-E-73767, ADP145172, female), PNN Chiribiquete, Rio Cuñare, $00^{\circ} 30^{\prime} \mathrm{N}, 072^{\circ} 37^{\prime} \mathrm{W}, 300 \mathrm{~m}, 1-4$ November 2000 (E González, M Ospina)(IAvH: IAvH-E-73760, ADP145144, female), 1-5 November 2000 (E González, M Ospina)(IAvH: IAvH-E-73768, ADP145150, male), MetA, 8 


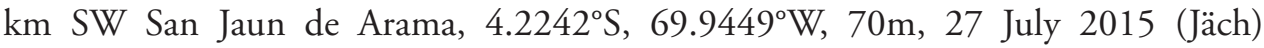
(NMNH, ADP140534, female), NARIÑo, Territoio Kofan, Orito, $0.499^{\circ} \mathrm{N}$, 77.216² W, 700m, 28 September 1998 (E González)(IAvH: IAvH-E-2440, ADP 145146, male). Ecuador, Napo, Tena, $0.989^{\circ} \mathrm{S}, 77.812^{\circ} \mathrm{W}, 500 \mathrm{~m}, 22$-27 May 1987 (BV Brown)(LACM: ADP006159, male), Orellana, Reserva Ethnica Huaorani, 39 km S Pompeya, Estación Científica Yasuní - Onkone Gare Camp, Erwin Piraña Plot: transect 6 , station $2,0.6561^{\circ} \mathrm{S}, 76.4483^{\circ} \mathrm{W}, 220-250 \mathrm{~m}, 15$ January 1994 (TL Erwin, et al.)(NMNH: ADP138234, male), Erwin Pirańa Plot: transect 4, station $8,0.6570^{\circ} \mathrm{S}, 76.4498^{\circ} \mathrm{W}, 220-250 \mathrm{~m}, 16$ January 1994 (TL Erwin, et al.) (NMNH: ADP135890, female), Erwin Pirańa Plot: transect 7, station 9, $0.6556^{\circ} \mathrm{S}$, $76.4475^{\circ} \mathrm{W}, 220-250 \mathrm{~m}, 20$ January 1994 (TL Erwin, et al.)(NMNH: ADP135903, male), Erwin Piraña Plot: transect 7, station 6, 0.6556 $6^{\circ}, 76.4475^{\circ} \mathrm{W}, 220-250 \mathrm{~m}, 20$ January 1994 (TL Erwin, et al.)(NMNH: ADP135905, males), Erwin Piraña Plot: transect 8 , station $1,0.6551^{\circ} \mathrm{S}, 76.4403^{\circ} \mathrm{W}, 220-250 \mathrm{~m}, 24$ January 1994 (TL Erwin, et al.)(NMNH: ADP138676, male), Erwin Piraña Plot: transect 8, station 6, $0.6551^{\circ} \mathrm{S}, 76.4403^{\circ} \mathrm{W}, 220-250 \mathrm{~m}, 24$ January 1994 (TL Erwin, et al.)(NMNH: ADP135960, male), Erwin Piraña Plot: transect 4, station 9, $0.6570^{\circ} \mathrm{S}, 76.4498^{\circ} \mathrm{W}$, 220-250m, 25 June 1994 (TL Erwin, et al.)(NMNH: ADP135968, female), Erwin Pirańa Plot: transect 5, station $10,0.6566^{\circ} \mathrm{S}, 76.4490^{\circ} \mathrm{W}, 220-250 \mathrm{~m}, 25$ June 1994 (TL Erwin, et al.)(NMNH: ADP135978, male), Erwin Pirańa Plot: transect 2, station 2, $0.6556^{\circ} \mathrm{S}, 76.4475^{\circ} \mathrm{W}, 220-250 \mathrm{~m}, 20$ June 1994 (TL Erwin, et al.)(NMNH: ADP135899, ADP135984, males), Erwin Pirańa Plot: transect 10, station 6, $0.6540^{\circ} \mathrm{S}, 76.4453^{\circ} \mathrm{W}, 220-250 \mathrm{~m}, 29$ June 1994 (TL Erwin, et al.)(NMNH: ADP135958, male), Erwin Piraña Plot: transect 8, station 5, $0.6551^{\circ} \mathrm{S}, 76.4403^{\circ} \mathrm{W}$, 220-250m, 21 June 1994 (TL Erwin, et al.)(NMNH: ADP135956, male), Erwin Pirańa Plot: transect 9, station 9, $0.6545^{\circ} \mathrm{S}, 76.4460^{\circ} \mathrm{W}, 220-250 \mathrm{~m}, 21$ June 1994 (TL Erwin, et al.)(NMNH: ADP135909, female), Erwin Pirańa Plot: transect 10, station $10,0.6540^{\circ} \mathrm{S}, 76.4453^{\circ} \mathrm{W}, 220-250 \mathrm{~m}, 29$ June 1994 (TL Erwin, et al.) (NMNH: ADP135904, female), Erwin Pirańa Plot: transect 1, station 10, $0.6586^{\circ} \mathrm{S}$, $76.4521^{\circ} \mathrm{W}, 220-250 \mathrm{~m}, 3$ July 1994 (TL Erwin, et al.)(NMNH: ADP135791, male, ADP 135856 , female), Erwin Pirańa Plot: transect 1 , station $8,0.6586^{\circ} \mathrm{S}, 76.4521^{\circ} \mathrm{W}$, 220-250m, 4 October 1994 (TL Erwin, et al.)(NMNH: ADP135895, female), Erwin Pirańa Plot: transect 2 station 7, 0.6581 ${ }^{\circ} \mathrm{S}, 76.4513^{\circ} \mathrm{W}, 220-250 \mathrm{~m}, 4$ October 1994 (TL Erwin, et al.)(NMNH: ADP135893, male), Erwin Pirańa Plot: transect 10, station $10,0.6540^{\circ} \mathrm{S}, 76.4433^{\circ} \mathrm{W}, 220-250 \mathrm{~m}, 6$ October 1994 (TL Erwin, et al.) (NMNH: ADP137733, female), Erwin Pirańa Plot: transect 8, station 10, $0.6551^{\circ} \mathrm{S}$, $76.4403^{\circ} \mathrm{W}, 220-250 \mathrm{~m}, 7$ October 1994 (TL Erwin, et al.)(NMNH: ADP006160, females), Erwin Piraña Plot: transect 5, station 7, 0.6566 ${ }^{\circ}$, $76.4490^{\circ} \mathrm{W}, 220-250 \mathrm{~m}$, 9 October 1994 (TL Erwin, et al.)(NMNH: ADP135907, female), Erwin Piraña Plot: transect 4 , station $10,0.6570^{\circ} \mathrm{S}, 76.4498^{\circ} \mathrm{W}, 220-250 \mathrm{~m}, 10$ October 1994 (TL Erwin, et al.)(NMNH: ADP137736, female), Erwin Piraña Plot: transect 9, station 4, $0.6545^{\circ} \mathrm{S}, 76.4460^{\circ} \mathrm{W}, 220-250 \mathrm{~m}, 8$ February 1995 (TL Erwin, et al.)(NMNH: ADP 135972, male), Erwin Piraña Plot: transect 9, station 10, $0.6545^{\circ} \mathrm{S}, 76.4460^{\circ} \mathrm{W}$, 
220-250m, 8 February 1995 (TL Erwin, et al.)(NMNH: ADP135944, male, ADP 135936 , female), Erwin Piraña Plot: transect 10, station 3, $0.6540^{\circ} \mathrm{S}, 76.4473^{\circ} \mathrm{W}$, 220-250m, 8 February 1995 (TL Erwin, et al.)(NMNH: ADP135942, male), Erwin Piraña Plot: transect 2, station 8, $0.6581^{\circ} \mathrm{S}, 76.4513^{\circ} \mathrm{W}, 220-250 \mathrm{~m}, 9$ February 1995 (TL Erwin, et al.)(NMNH: ADP137846, female), Erwin Piraña Plot: transect 6, station $10,0.6561^{\circ} \mathrm{S}, 76.4483^{\circ} \mathrm{W}, 220-250 \mathrm{~m}, 9$ February 1995 (TL Erwin, et al.) (NMNH: ADP137357, female), Erwin Pirańa Plot: transect 7, station 8, $0.6556^{\circ} \mathrm{S}$, $76.4475^{\circ} \mathrm{W}, 220-250 \mathrm{~m}, 10$ February 1995 (TL Erwin, et al.)(NMNH: ADP137740, male), Erwin Piraña Plot: transect 4, station 2, 0.6566 ${ }^{\circ}, 76.4490^{\circ} \mathrm{W}, 220-250 \mathrm{~m}, 11$ February 1995 (TL Erwin, et al.)(NMNH: ADP137379, male, ADP137339, female), Erwin Pirańa Plot: transect 4, station 7, $0.6570^{\circ} \mathrm{S}, 76.4498^{\circ} \mathrm{W}, 220-250 \mathrm{~m}, 3$ July 1995 (TL Erwin, et al. (NMNH: ADP006172, female), Erwin Pirańa Plot: transect 3, station 9, $0.6566^{\circ} \mathrm{S}, 76.4490^{\circ} \mathrm{W}, 220-250 \mathrm{~m}, 6$ October 1995 (TL Erwin, et al.) (NMNH: ADP135952, male), Erwin Piraña Plot: transect 5, station 7, $0.6566^{\circ} \mathrm{S}$, 76.4490 W, 220-250m, 6 October 1995 (TL Erwin, et al.)(NMNH: ADP137325, ADP137373, males, ADP137365, female), Erwin Piraña Plot: transect 6, station 1, $0.6561^{\circ} \mathrm{S}, 76.4483^{\circ} \mathrm{W}, 220-250 \mathrm{~m}, 6$ October $^{1995}$ (TL Erwin, et al.)(NMNH: ADP 137327 , female), Erwin Pirańa Plot: transect 6 , station $6,0.6561^{\circ} \mathrm{S}, 76.4483^{\circ} \mathrm{W}$, 220-250m, 6 October 1995 (TL Erwin, et al.)(NMNH: ADP137391, female), Erwin Piraña Plot: transect 6 , station $10,0.6561^{\circ} \mathrm{S}, 76.44838^{\circ} \mathrm{W}, 220-250 \mathrm{~m}, 6$ October 1995 (TL Erwin, et al.)(NMNH: ADP137375, female), Erwin Piraña Plot: transect 8, station 7, $0.6551^{\circ} \mathrm{S}, 76.4403^{\circ} \mathrm{W}, 220-250 \mathrm{~m}, 7$ October 1995 (TL Erwin, et al.) (NMNH: ADP135988, female), Erwin Piraña Plot: transect 9, station 8, $0.6545^{\circ} \mathrm{S}$, $76.4460^{\circ} \mathrm{W}, 220-250 \mathrm{~m}, 8$ October 1995 (TL Erwin, et al.)(NMNH: ADP135982, male), Erwin Piraña Plot: transect 9, station 9, $0.6545^{\circ} \mathrm{S}, 76.4460^{\circ} \mathrm{W}, 220-250 \mathrm{~m}, 8$ October 1995 (TL Erwin, et al.)(NMNH: ADP135946, male), Erwin Pirańa Plot: transect 10, station $2,0.6540^{\circ} \mathrm{S}, 76.4453^{\circ} \mathrm{W}, 220-250 \mathrm{~m}, 8$ October 1995 (TL Erwin, et al.)(NMNH: ADP135938, male, ADP135948, female), Erwin Piraña Plot: transect 10 , station $4,0.6540^{\circ} \mathrm{S}, 76.4453^{\circ} \mathrm{W}, 220-250 \mathrm{~m}, 8$ October 1995 (TL Erwin, et al.)(NMNH: ADP135966, male), Erwin Piraña Plot: transect 10, station 5, $0.6540^{\circ} \mathrm{S}$, $76.4453^{\circ} \mathrm{W}, 220-250 \mathrm{~m}, 8$ October 1995 (TL Erwin, et al.)(NMNH: ADP135964, female), Erwin Piraña Plot: transect 2, station 3, 0.6581 ${ }^{\circ} \mathrm{S}, 76.4513^{\circ} \mathrm{W}, 220-250 \mathrm{~m}, 9$ October 1995 (TL Erwin, et al.)(NMNH: ADP135915, male), Erwin Pirańa Plot: transect 2 , station $7,0.6581^{\circ} \mathrm{S}, 76.4513^{\circ} \mathrm{W}, 220-250 \mathrm{~m}$, 4 February 1996 (TL Erwin, et al.)(NMNH: ADP137696, male), Erwin Piraña Plot: transect 2, station 8, $0.6581^{\circ} \mathrm{S}, 76.4513^{\circ} \mathrm{W}, 220-250 \mathrm{~m}, 4$ February 1996 (TL Erwin, et al.)(NMNH: ADP135980, female), Erwin Piraña Plot: transect 4, station 6, $0.6576^{\circ} \mathrm{S}, 76.4498^{\circ} \mathrm{W}$, 220-250m, 5 February 1996 (TL Erwin, et al.)(NMNH: ADP136473, male), Erwin Pirańa Plot: transect 6, station 8, 0.6561 ${ }^{\circ} \mathrm{S}, 76.4483^{\circ} \mathrm{W}, 220-250 \mathrm{~m}, 7$ February 1996 (TL Erwin, et al.)(NMNH: ADP137329, female), Erwin Piraña Plot: transect 10, $0.6586^{\circ} \mathrm{S}, 76.4521^{\circ} \mathrm{W}, 220-250 \mathrm{~m}, 13$ February 1996 (TL Erwin, et al.)(NMNH: ADP147731, male), Erwin Pirańa Plot: transect 4, station 7, $0.6570^{\circ} \mathrm{S}, 76.4498^{\circ} \mathrm{W}$, 220-250m, 21 June 1996 (TL Erwin, et al.)(NMNH: ADP137363, male), Erwin 
Piraña Plot: transect 9, station 4, 0.6570 ${ }^{\circ} \mathrm{S}, 76.4498^{\circ} \mathrm{W}, 220-250 \mathrm{~m}, 21$ June 1996 (TL Erwin, et al.)(NMNH: ADP137343, male), Erwin Piraña Plot: transect 5, station 3, $0.6566^{\circ} \mathrm{S}, 76.4490^{\circ} \mathrm{W}, 220-250 \mathrm{~m}, 22$ June 1996 (TL Erwin, et al.)(NMNH: ADP137345, male), Erwin Piraña Plot: transect 5, station 9, $0.6566^{\circ} \mathrm{S}, 76.4490^{\circ} \mathrm{W}$, 220-250m, 22 June 1996 (TL Erwin, et al.)(NMNH: ADP137347, male), Erwin Pirańa Plot: transect 6, station 9, 0.6561 ${ }^{\circ} \mathrm{S}, 76.4483^{\circ} \mathrm{W}, 220-250 \mathrm{~m}, 22$ June 1996 (TL Erwin, et al.)(NMNH: ADP137688, female), Erwin Pirańa Plot: transect 9, station 7, 0.6545 ${ }^{\circ} \mathrm{S}, 76.4460^{\circ} \mathrm{W}, 220-250 \mathrm{~m}, 23$ June 1996 (TL Erwin, et al.)(NMNH: ADP137744, male, ADP137742, female), Erwin Pirańa Plot: transect 9, station 9, $0.6595^{\circ} \mathrm{S}, 76.4460^{\circ} \mathrm{W}, 220-250 \mathrm{~m}, 23$ June 1996 (TL Erwin, et al.)(NMNH: ADP137734, male), Erwin Pirańa Plot: transect 1, station 3, $0.6586^{\circ} \mathrm{S}, 76.4521^{\circ} \mathrm{W}$, 220-250m, 25 June 1996 (TL Erwin, et al.)(NMNH: ADP137748, male), Erwin Pirańa Plot: transect 1 , station $10,0.6586^{\circ} \mathrm{S}, 76.4521^{\circ} \mathrm{W}, 220-250 \mathrm{~m}, 30$ September 1996 (TL Erwin, et al.)(NMNH: ADP137331, ADP137359, males), Erwin Piraña Plot: transect 2 , station $7,0.6581^{\circ} \mathrm{S}, 76.4513^{\circ} \mathrm{W}, 220-250 \mathrm{~m}, 30$ September 1996 (TL Erwin, et al.)(NMNH: ADP137361, female), Erwin Piraña Plot: transect 8, station 7, $0.6561^{\circ} \mathrm{S}, 76.4483^{\circ} \mathrm{W}, 220-250 \mathrm{~m}, 2$ October 1996 (TL Erwin, et al.)(NMNH: ADP135928, male), Erwin Pirańa Plot: transect 7, station 3, $0.6566^{\circ} \mathrm{S}, 76.4490^{\circ} \mathrm{W}$, 220-250m, 3 October 1996 (TL Erwin, et al.)(NMNH: ADP135913, male), Erwin Piraña Plot: transect 8, station 5, 0.6551 ${ }^{\circ} \mathrm{S}, 76.4403^{\circ} \mathrm{W}, 220-250 \mathrm{~m}, 3$ October 1996 (TL Erwin, et al.)(NMNH: ADP135920, male), Erwin Piraña Plot: transect 8, station 10, $0.6551^{\circ} \mathrm{S}, 76.4403^{\circ} \mathrm{W}, 220-250 \mathrm{~m}, 3$ October 1996 (TL Erwin, et al.) (NMNH: ADP137385, male, ADP137367, female), Erwin Piraña Plot: transect 9, station 5, $0.6545^{\circ} \mathrm{S}, 76.4460^{\circ} \mathrm{W}, 220-250 \mathrm{~m}, 4$ October 1996 (TL Erwin, et al.) (NMNH: ADP137351, female), Erwin Piraña Plot: transect 5, station 2, $0.6566^{\circ} \mathrm{S}$, 76.4490 W, 220-250m, 6 October 1996 (TL Erwin, et al.)(NMNH: ADP135862, female), Erwin Pirańa Plot: transect 4, station 7, 0.6570 ${ }^{\circ} \mathrm{S}, 76.4498^{\circ} \mathrm{W}, 220-250 \mathrm{~m}$, 20 January 2006 (TL Erwin, et al.)(NMNH: ADP133848, female), Erwin Piraña Plot: transect 5 , station $1,0.6566^{\circ} \mathrm{S}, 76.4490^{\circ} \mathrm{W}, 220-250 \mathrm{~m}, 20$ January 2006 (TL Erwin, et al.)(NMNH: ADP133854, female), Erwin Piraña Plot: transect 8, station 4, $0.6556^{\circ} \mathrm{S}, 76.4475^{\circ} \mathrm{W}, 220-250 \mathrm{~m}, 28$ January 2006 (TL Erwin, et al.)(NMNH: ADP133866, male), Erwin Pirańa Plot: transect 1, station $1,0.6586^{\circ} \mathrm{S}, 76.4421^{\circ} \mathrm{W}$, 220-250m, 7 July 2006 (TL Erwin, et al.)(NMNH: ADP133856, male), Erwin Piraña Plot: transect 1 , station 2, $0.6586^{\circ} \mathrm{S}, 76.4421^{\circ} \mathrm{W}, 220-250 \mathrm{~m}, 7$ July 2006 (TL Erwin, et al.)(NMNH: ADP133858, male), Erwin Pirańa Plot: transect 6, station 7, $0.6561^{\circ} \mathrm{S}, 76.4483^{\circ} \mathrm{W}, 220-250 \mathrm{~m}, 9$ July 2006 (TL Erwin, et al.)(NMNH: ADP133850, male), Erwin Pirańa Plot: transect 9, station 5, $0.6545^{\circ} \mathrm{S}, 76.4460^{\circ} \mathrm{W}$, 220-250m, 12 July 2006 (TL Erwin, et al.)(NMNH: ADP133862, male), Yasuni National Park (edge), $95.43 \mathrm{~km}$ E (heading 101.46 ${ }^{\circ}$ Coca, Tiputini Biodiversity Station, Erwin Harpia Plot: transect 3, station 9, 0.63327 $\mathrm{S}, 76.1443^{\circ} \mathrm{W}, 207 \mathrm{~m}, 20$ June 1998 (TL Erwin, et al.)(NMNH: ADP135860, ADP135906, females), Erwin Harpia Plot: transect 4 , station $8,0.63167^{\circ} \mathrm{S}, 76.1443^{\circ} \mathrm{W}, 208 \mathrm{~m}, 30$ June 1998 (TL Erwin, 
et al.)(NMNH: ADP135795, male), Erwin Harpia Plot: transect 6, station 10, $0.6295^{\circ} \mathrm{S}, 76.1443^{\circ} \mathrm{W}, 199 \mathrm{~m}, 1$ July 1998 (TL Erwin, et al.)(NMNH: ADP135834, male), Erwin Harpia Plot: transect 7 , station $10,0.6287^{\circ} \mathrm{S}, 76.1443^{\circ} \mathrm{W}, 203 \mathrm{~m}, 4$ July 1998 (TL Erwin, et al.)(NMNH: ADP135868, male), Erwin Harpia Plot: transect 8, station 5, $0.6278^{\circ} \mathrm{S}, 76.1443^{\circ} \mathrm{W}, 203 \mathrm{~m}, 4$ July 1998 (TL Erwin, et al.)(NMNH: ADP 135910, male), Erwin Harpia Plot: transect 9, station 7, $0.6269^{\circ} \mathrm{S}, 76.1443^{\circ} \mathrm{W}$, 203m, 5 July 1998 (TL Erwin, et al.)(NMNH: ADP135841, male), Erwin Harpia Plot: transect 10 , station $6,0.6262^{\circ} \mathrm{S}, 76.1443^{\circ} \mathrm{W}, 214 \mathrm{~m}, 5$ July 1998 (TL Erwin, et al.)(NMNH: ADP 135854, male), Erwin Harpia Plot: transect 10, station 8, $0.6287^{\circ} \mathrm{S}$, $76.1443^{\circ} \mathrm{W}, 214 \mathrm{~m}, 5$ July 1998 (TL Erwin, et al.)(NMNH: ADP135838, male), Erwin Harpia Plot: transect 9, station 6, 0.6269 ${ }^{\circ}$ S, $76.1443^{\circ} \mathrm{W}, 203 \mathrm{~m}, 21$ October 1998 (TL Erwin, et al.)(NMNH: ADP136756, female), Erwin Harpia Plot: transect 9, station 9, $0.6269^{\circ} \mathrm{S}, 76.1443^{\circ} \mathrm{W}, 203 \mathrm{~m}, 21$ October 1998 (TL Erwin, et al.) (NMNH: ADP135866, male), Erwin Harpia Plot: transect 10, station 3, $0.6262^{\circ} \mathrm{S}$, $76.1443^{\circ} \mathrm{W}, 214 \mathrm{~m}, 21$ October $_{1998}$ (TL Erwin, et al.)(NMNH: ADP135882, female), Erwin Harpia Plot: transect 10, station 6, 0.6262 ${ }^{\circ}, 76.1443^{\circ} \mathrm{W}, 215 \mathrm{~m}, 21$ October 1998 (TL Erwin, et al.)(NMNH: ADP135846, male), Erwin Harpia Plot: transect 7 , station $1,0.6287^{\circ} \mathrm{S}, 76.1443^{\circ} \mathrm{W}, 203 \mathrm{~m}, 22$ October 1998 (TL Erwin, et al.)(NMNH: ADP135884, male, ADP135888, female), Erwin Harpia Plot: transect 8 , station $8,0.6273^{\circ} \mathrm{S}, 76.1443^{\circ} \mathrm{W}, 203 \mathrm{~m}, 22$ October 1998 (TL Erwin, et al.) (NMNH: ADP136798, male), Erwin Harpia Plot: transect 8, station 10, $0.6287^{\circ} \mathrm{S}$, $76.1443^{\circ} \mathrm{W}, 203 \mathrm{~m}, 22$ October 1998 (TL Erwin, et al.)(NMNH: ADP135864, female), Erwin Harpia Plot: transect 2, station 4, $0.6332^{\circ} \mathrm{S}, 76.1443^{\circ} \mathrm{W}, 197 \mathrm{~m}, 23$ October 1998 (TL Erwin, et al.)(NMNH: ADP135839, male), Erwin Harpia Plot: transect 2, station $8,0.6332^{\circ} \mathrm{S}, 76.1443^{\circ} \mathrm{W}, 197 \mathrm{~m}, 23$ October 1998 (TL Erwin, et al.)(NMNH: ADP135880, female), Erwin Harpia Plot: transect 3, station 2, $0.6332^{\circ} \mathrm{S}, 76.1443^{\circ} \mathrm{W}, 207 \mathrm{~m}, 24$ October 1998 (TL Erwin, et al.)(NMNH: ADP135878, male), Erwin Harpia Plot: transect 3, station 3, $0.6332^{\circ} \mathrm{S}, 76.1443^{\circ} \mathrm{W}$, 207m, 24 October 1998 (TL Erwin, et al.)(NMNH: ADP135769, male), Erwin Harpia Plot: transect 3, station 4, $0.6332^{\circ} \mathrm{S}, 76.1443^{\circ} \mathrm{W}, 207 \mathrm{~m}, 24$ October 1998 (TL Erwin, et al.)(NMNH: ADP135892, male), Erwin Harpia Plot: transect 3, station $9,0.6332^{\circ} \mathrm{S}, 76.1443^{\circ} \mathrm{W}, 207 \mathrm{~m}, 24$ October 1998 (TL Erwin, et al.)(NMNH: ADP 135912 , female), Erwin Harpia Plot: transect 4, station $5,0.6316^{\circ} \mathrm{S}, 76.1443^{\circ} \mathrm{W}$, 208m, 24 October 1998 (TL Erwin, et al.)(NMNH: ADP137335, male, ADP135872, female), Erwin Harpia Plot: transect 6, station 5, 0.6295 S, 76.1443 ${ }^{\circ} \mathrm{W}, 199 \mathrm{~m}, 26$ October 1998 (TL Erwin, et al.)(NMNH: ADP135837, male), Erwin Harpia Plot: transect 5, station $2,0.6304^{\circ} \mathrm{S}, 76.1443^{\circ} \mathrm{W}, 209 \mathrm{~m}, 2$ February 1999 (TL Erwin, et al.)(NMNH: ADP139104, female), Erwin Harpia Plot: transect 10, station 6, $0.6262^{\circ} \mathrm{S}, 76.1443^{\circ} \mathrm{W}, 214 \mathrm{~m}, 5$ February 1999 (TL Erwin, et al.)(NMNH: ADP135886, male), Erwin Harpia Plot: transect 10, station 7, $0.6262^{\circ} \mathrm{S}, 76.1443^{\circ} \mathrm{W}$, 214m, 5 February 1999 (TL Erwin, et al.)(NMNH: ADP135815, male), Erwin Harpia Plot: transect 7 , station 3, $0.6287^{\circ} \mathrm{S}, 76.1443^{\circ} \mathrm{W}, 203 \mathrm{~m}, 6$ February 1999 (TL 
Erwin, et al.)(NMNH: ADP135876, female), Erwin Harpia Plot: transect 8, station 4, $0.6278^{\circ} \mathrm{S}, 76.1443^{\circ} \mathrm{W}, 203 \mathrm{~m}, 6$ February 1999 (TL Erwin, et al.)(NMNH: ADP135842, female), Erwin Harpia Plot: transect 5, station $7,0.6304^{\circ} \mathrm{S}, 76.1443^{\circ} \mathrm{W}$, 209m, 7 February 1999 (TL Erwin, et al.)(NMNH: ADP135817, male), Erwin Harpia Plot: transect 6, station 2, 0.6295 S, 76.1443 W, 199m, 7 February 1999 (TL Erwin, et al.)(NMNH: ADP135914, female), Erwin Harpia Plot: transect 6, station 6, $0.6295^{\circ} \mathrm{S}, 76.1443^{\circ} \mathrm{W}, 199 \mathrm{~m}, 7$ February 1999 (TL Erwin, et al.)(NMNH: ADP 135894, female), Erwin Harpia Plot: transect 4, station 6, 0.6316 ${ }^{\circ} \mathrm{S}, 76.1443^{\circ} \mathrm{W}$, 208m, 8 February 1999 (TL Erwin, et al.)(NMNH: ADP135852, female), Erwin Harpia Plot: transect 1, station 2, 0.6342 ${ }^{\circ} \mathrm{S}, 76.1443^{\circ} \mathrm{W}, 218 \mathrm{~m}, 9$ February 1999 (TL Erwin, et al.)(NMNH: ADP135858, male), Erwin Harpia Plot: transect 1, station 3, $0.6332^{\circ} \mathrm{S}, 76.1443^{\circ} \mathrm{W}, 207 \mathrm{~m}, 21$ October 1999 (TL Erwin, et al.)(NMNH: ADP135918, female), Erwin Harpia Plot: transect 10, station 5, $0.6262^{\circ} \mathrm{S}, 76.1443^{\circ} \mathrm{W}$, 214m, 22 October 1999 (TL Erwin, et al.)(NMNH: ADP135901, male), Erwin Harpia Plot: transect 10, station 6, 0.6262 ${ }^{\circ}$, 76.1443 ${ }^{\circ} \mathrm{W}, 214 \mathrm{~m}, 22$ October 1999 (TL Erwin, et al.)(NMNH: ADP137694, male), Erwin Harpia Plot: transect 3, station $8,0.6232^{\circ} \mathrm{S}, 76.1443^{\circ} \mathrm{W}, 207 \mathrm{~m}, 23$ October 1999 (TL Erwin, et al.)(NMNH: ADP139521, male), Erwin Harpia Plot: transect 6, station 9, 0.6287 $\mathrm{S}, 76.1443^{\circ} \mathrm{W}$, 199m, 24 October 1999 (TL Erwin, et al.)(NMNH: ADP137754, male), Erwin Harpia Plot: transect 6, station 3, 0.6295 S, 76.1443 W, 199m, 25 October 1999 (TL Erwin, et al.)(NMNH: ADP137758, female), Erwin Harpia Plot: transect 9, station $8,0.6269^{\circ} \mathrm{S}, 76.1443^{\circ} \mathrm{W}, 203 \mathrm{~m}, 28$ September 2000 (TL Erwin, et al.)(NMNH: ADP139152, female), Erwin Harpia Plot: transect 9, station 9, $0.6269^{\circ} \mathrm{S}, 76.1443^{\circ} \mathrm{W}$, 203m, 28 September 2000 (TL Erwin, et al.)(NMNH: ADP139160, male), Erwin Harpia Plot: transect 10, station 4, $0.6262^{\circ} \mathrm{S}, 76.1443^{\circ} \mathrm{W}, 214 \mathrm{~m}, 28$ September 2000 (TL Erwin, et al.)(NMNH: ADP139154, ADP139142, ADP139144, males), Erwin Harpia Plot: transect 4, station 5, 0.6316 ${ }^{\circ}$, $76.1443^{\circ} \mathrm{W}, 208 \mathrm{~m}, 1$ October 2000 (TL Erwin, et al.)(NMNH: ADP139162, female), Erwin Harpia Plot: transect 4, station 6, $0.6316^{\circ} \mathrm{S}, 76.1443^{\circ} \mathrm{W}, 208 \mathrm{~m}, 1$ October 2000 (TL Erwin, et al.)(NMNH: ADP139158, male), Erwin Harpia Plot: transect 5, station 3, $0.6316^{\circ} \mathrm{S}, 76.1443^{\circ} \mathrm{W}$, 214m, 1 October 2000 (TL Erwin, et al.)(NMNH: ADP139136, female), Erwin Harpia Plot: transect 6, station $1,0.6304^{\circ} \mathrm{S}, 76.1443^{\circ} \mathrm{W}, 199 \mathrm{~m}, 1$ October 2000 (TL Erwin, et al.)(NMNH: ADP139126, female), Erwin Harpia Plot: transect 6, station 3, $0.6304^{\circ} \mathrm{S}, 76.1443^{\circ} \mathrm{W}, 199 \mathrm{~m}, 1$ October 2000 (TL Erwin, et al.)(NMNH: ADP139092, ADP139164, females), Erwin Harpia Plot: transect 6, station 5, $0.6295^{\circ} \mathrm{S}, 76.1443^{\circ} \mathrm{W}, 199 \mathrm{~m}, 1$ October 2000 (TL Erwin, et al.)(NMNH: ADP139130, male), Erwin Harpia Plot: transect 1, station 3, 0.6316 ${ }^{\circ} \mathrm{S}, 76.1443^{\circ} \mathrm{W}$, 214m, 2 October 2000 (TL Erwin, et al.)(NMNH: ADP139122, male), Erwin Harpia Plot: transect 1, station 5, 0.6342 ${ }^{\circ} \mathrm{S}, 76.1443^{\circ} \mathrm{W}, 214 \mathrm{~m}, 2$ October 2000 (TL Erwin, et al.)(NMNH: ADP139146, female), Erwin Harpia Plot: transect 1, station 6, $0.6342^{\circ} \mathrm{S}, 76.1443^{\circ} \mathrm{W}, 214 \mathrm{~m}, 2$ October 2000 (TL Erwin, et al.)(NMNH: ADP139094, male), Erwin Harpia Plot: transect 6, station 1, 0.6342 ${ }^{\circ} \mathrm{S}, 76.1443^{\circ} \mathrm{W}$, 
214m, 2 October 2000 (TL Erwin, et al.)(NMNH: ADP139096, female), Erwin Harpia Plot: transect 3, station $10,0.6330^{\circ} \mathrm{S}, 76.1443^{\circ} \mathrm{W}, 207 \mathrm{~m}, 4$ October 2000 (TL Erwin, et al.)(NMNH: ADP139148, female), Erwin Harpia Plot: transect 10, station 4, $0.6332^{\circ} \mathrm{S}, 76.1443^{\circ} \mathrm{W}, 207 \mathrm{~m}, 4$ October 2000 (TL Erwin, et al.)(NMNH: ADP139156, male), Erwin Harpia Plot: transect 1, station 4, $0.6342^{\circ} \mathrm{S}, 76.1443^{\circ} \mathrm{W}$, 214m, 16 February 2001 (TL Erwin, et al.)(NMNH: ADP139114, female), Erwin Harpia Plot: transect 2, station 2, $0.6332^{\circ} \mathrm{S}, 76.1443^{\circ} \mathrm{W}, 197 \mathrm{~m}, 16$ February 2001 (TL Erwin, et al.)(NMNH: ADP139110, male), Erwin Harpia Plot: transect 9, station $7,0.6269^{\circ} \mathrm{S}, 76.1443^{\circ} \mathrm{W}, 203 \mathrm{~m}, 17$ February 2001 (TL Erwin, et al.)(NMNH: ADP139116, female), Erwin Harpia Plot: transect 9, station 8, $0.6269^{\circ} \mathrm{S}, 76.1443^{\circ} \mathrm{W}$, 203m, 17 February 2001 (TL Erwin, et al.)(NMNH: ADP139102, female), Erwin Harpia Plot: transect 9, station 9, $0.6268^{\circ}$ S, 76.1443ํ. $203 \mathrm{~m}, 17$ February 2001 (TL Erwin, et al.)(NMNH: ADP139120, male, ADP139118, female), Erwin Harpia Plot: transect 1 , station $2,0.6342^{\circ} \mathrm{S}, 76.1443^{\circ} \mathrm{W}, 214 \mathrm{~m}, 19$ July 2001 (TL Erwin, et al.)(NMNH: ADP139463, female), Erwin Harpia Plot: transect 1, station 3, $0.6342^{\circ} \mathrm{S}, 76.1443^{\circ} \mathrm{W}, 214 \mathrm{~m}, 19$ July 2001 (TL Erwin, et al.)(NMNH: ADP139469, male), Erwin Harpia Plot: transect 1, station 8, $0.6342^{\circ} \mathrm{S}, 76.1443^{\circ} \mathrm{W}, 214 \mathrm{~m}, 19$ July 2001 (TL Erwin, et al.)(NMNH: ADP139106, male), Erwin Harpia Plot: transect 10 , station $1,0.6262^{\circ} \mathrm{S}, 76.1443^{\circ} \mathrm{W}, 214 \mathrm{~m}, 20$ July 2001 (TL Erwin, et al.)(NMNH: ADP139523, male), Erwin Harpia Plot: transect 10, station 3, $0.6269^{\circ} \mathrm{S}, 76.1443^{\circ} \mathrm{W}$, 214m, 20 July 2001 (TL Erwin, et al.)(NMNH: ADP139465, male), Erwin Harpia Plot: transect 9, station 7, $0.6269^{\circ} \mathrm{S}, 76.1443^{\circ} \mathrm{W}, 203 \mathrm{~m}, 20$ July 2001 (TL Erwin, et al.) (NMNH: ADP139467, male), Erwin Harpia Plot: transect 10, station 4, $0.6262^{\circ} \mathrm{S}$, $76.1443^{\circ} \mathrm{W}, 214 \mathrm{~m}, 20$ July 2001 (TL Erwin, et al.)(NMNH: ADP139525, male), Erwin Harpia Plot: transect 3, station 1, $0.6332^{\circ} \mathrm{S}, 76.1443^{\circ} \mathrm{W}, 207 \mathrm{~m}, 24$ July 2001 (TL Erwin, et al.)(NMNH: ADP139461, male); Sucumbíos, Río Napo, Sacha Lodge, Pilchicocha, $0.472^{\circ} \mathrm{S}, 76.459^{\circ} \mathrm{W}, 228 \mathrm{~m}, 12-22$ February 1994 (P Hibbs) (SEMC: ADP006158, female) Río Napo, Sacha Lodge, Pilchicocha, $0.472^{\circ} \mathrm{S}$, 76.459 ${ }^{\circ} \mathrm{W}, 228 \mathrm{~m}, 22$ February - 4 March 1994 (P Hibbs)(SEMC: ADP006156) Río Napo, Sacha Lodge, Pilchicocha, 0.472 ${ }^{\circ}$ S, 76.459 $\mathrm{W}, 228 \mathrm{~m}, 4-14$ March 1994 (P Hibbs)(SEMC: ADP006112, ADP006131, ADP006155, females) Río Napo, Sacha Lodge, Pilchicocha, $0.472^{\circ} \mathrm{S}, 76.459^{\circ} \mathrm{W}, 228 \mathrm{~m}, 14-24 \mathrm{March} 1994$ (P Hibbs)(SEMC: ADP006114, ADP006154, females) Río Napo, Sacha Lodge, Pilchicocha, $0.472^{\circ} \mathrm{S}$, 76.459 ${ }^{\circ} \mathrm{W}, 228 \mathrm{~m}, 3-13$ April 1994 (P Hibbs)(SEMC: ADP006137, male, ADP006128, ADP006136, females) Río Napo, Sacha Lodge, Pilchicocha, $0.472^{\circ} \mathrm{S}$, 76.459 ${ }^{\circ} \mathrm{W}, 228 \mathrm{~m}, 13-23$ April 1994 (P Hibbs)(SEMC: ADP006151, male, ADP006152, female) Río Napo, Sacha Lodge, Pilchicocha, $0.472^{\circ} \mathrm{S}, 76.459^{\circ} \mathrm{W}$, 228m, 23 April - 4 May 1994 (P Hibbs)(SEMC: ADP006129, ADP006134, males, ADP006113, ADP006135, female), 13-25 July 1994 (P Hibbs)(SEMC: ADP006115, male, ADP006153, female), 3-16 August 1994 (P Hibbs)(SEMC: ADP006157, male), 20-30 September 1994 (P Hibbs)(SEMC: ADP006130, ADP006150, females), Río Napo, Sacha Lodge, Pilchicocha, $0.472^{\circ} \mathrm{S}, 76.459^{\circ} \mathrm{W}, 228 \mathrm{~m}, 3-16$ August 
1994, P Hibbs, SEMC, 006138, female paratype) Limoncocha National Biological Reserve, Limoncocha, $0.4069^{\circ} \mathrm{S}, 76.6134^{\circ} \mathrm{W}, 235 \mathrm{~m}, 11$ June 1977 (PJ Spangler, D.R Givens)(NMNH: ADP005800, female). French Guiana, Cayenne, Mont Itoupe, placette, $3.0220^{\circ} \mathrm{N}, 53.0947^{\circ} \mathrm{W}, 600 \mathrm{~m}, 26$ November 2014 (S Brule, PH Dalens, E Poirier)(NMNH: ADP148089, female),16 January 2016 (S Brule, PH Dalens, E Poirier)(NMNH: ADP148830, ADP148831, females), 800m, 2 December 2014 (S Brule, PH Dalens, E Poirier)(NMNH: ADP148201, female); Commune de Roura, Montagne des Chevaux, $4.7127^{\circ} \mathrm{N}, 52.3966^{\circ} \mathrm{W}, 90,3$ January 2010 (S Brule, PH Dalens, E Poirier)(NMNH: ADP128009, female), 3 January 2015 (S Brule, PH Dalens, E Poirier)(NMNH: ADP148090, female), 1 August 2015 (S Brule, PH Dalens, E Poirier)(NMNH: ADP148101, male), 15 km west, Regina, Petit Montagne Tortue, $4.3204^{\circ} \mathrm{N}, 52.2404^{\circ} \mathrm{W}, 94 \mathrm{~m}, 10$ June 2010 (G Lamarre)(NMNH: ADP130780, male), Region de Saul, Commune de Saul, Belvedere de Saul, $3.6223^{\circ} \mathrm{N}$, $53.2159^{\circ} \mathrm{W}, 283-325 \mathrm{~m}, 5$ February 2010 (S Brule, PH Dalens, E Poirier)(NMNH: ADP130778, female), 9 September 2010 (S Brule, PH Dalens, E Poirier)(NMNH: ADP130777, female), 14 February 2011 (S Brule, PH Dalens, E Poirier)(NMNH: ADP134141, male), 11 December 2012 (S Brule, PH Dalens, E Poirier)(NMNH: ADP 134138, male), 16 January 2013 (S Brule, PH Dalens, E Poirier)(NMNH: ADP134136, male, ADP134139, female), 6 February 2013 (S Brule, PH Dalens, E Poirier)(NMNH: ADP134140, female) Region de Saul, Commune de Saul, Belvedere de Saul (point de vue), 3.6223N, 53.2159 ${ }^{\circ} \mathrm{W}, 283-325 \mathrm{~m}, 19$ August 2010 (S Brule, PH Dalens, E Poirier)(NMNH: ADP130779, female), 7 March 2011 (S Brule, PH Dalens, E Poirier)(NMNH: ADP135284, female), 11 December 2012 (S Brule, PH Dalens, E Poirier)(NMNH: ADP134137, female), Camp Inselberg, Nouragues, Commune de Regina, $4.0334^{\circ} \mathrm{N}, 52.6786^{\circ} \mathrm{W}, 411 \mathrm{~m}$, 5 September 2013 (S Brule, PH Dalens, E Poirier)(NMNH: ADP147737, female). Perú, Junin, Satipo, 11.265'S, $74.634^{\circ} \mathrm{W}, 865 \mathrm{~m}$, July 1941 (A. Maller)(AMNH: ADP007287, male paratype); Lo-

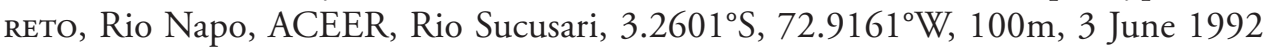
(TL Erwin, E Pfuno S, F Pfuno S)(NMNH: ADP052156, male, ADP052134, female), 15 June 1992 (TL Erwin, E Pfuno S, F Pfuno S)(NMNH: ADP052729, female), 11 June 1992 (TL Erwin, E Pfuno S, F Pfuno S)(NMNH: ADP051235, male, ADP052671, female), 15 June 1992 (TL Erwin, E Pfuno S, F Pfuno S)(NMNH: ADP052704, ADP052730, females), ACEER-Explonapo Camp, Rio Sucusari, $3.2601^{\circ} \mathrm{S}, 72.9161^{\circ} \mathrm{W}, 100 \mathrm{~m}, 3$ June 1992 (TL Erwin, E Pfuno S, F Pfuno S) (NMNH: ADP052134, female), 6 June 1992 (TL Erwin, E Pfuno S, F Pfuno S) (NMNH: ADP053140, male), Pacaya-Samiria National Reserve, Río Samiria, Cocha Shinguito, $5.1775^{\circ} \mathrm{S}, 76.6556^{\circ} \mathrm{W}, 112 \mathrm{~m}, 26$ May 1990 (TL Erwin, et al.)(NMNH: ADP067108, male, ADP067084, female), 27 May 1990 (D. Silva)(NMNH: ADP007573, female), 20 June 1990 (TL Erwin, et al.)(NMNH: ADP092866, male, ADP066883, ADP066937, females), 27 August 1991 (TL Erwin, MG Pogue, et al.) (NMNH: ADP050386, female), Río Samiria (South Branch), Camp Terry, 5.6951ํㄱ, $75.2243^{\circ} \mathrm{W}, 129 \mathrm{~m}, 14$ May 1990 (TL Erwin)(NMNH: ADP093061, male, ADP093060, female), $1 \mathrm{~km}$ E Hamburgo, Boca del Ingles Camp, $0.5226^{\circ} \mathrm{S}$, 
75.1192 $\mathrm{W}, 125 \mathrm{~m}, 20$ August 1991 (TL Erwin, MG Pogue)(NMNH: ADP070664, female), $1.5 \mathrm{~km} \mathrm{~N}$ Teniente Lopez, 2.3566 $\mathrm{S}, 76.0692^{\circ} \mathrm{W}, 210-240 \mathrm{~m}, 22$ July 1993 (R Leschen)(SEMC: ADP007288, female), nr. Iquitos, Porvenir, 3.8918 ${ }^{\circ} \mathrm{S}$, 73.5603ํW, 24 June 2011 (G. Lamarre)(NMNH: ADP135887, ADP135889, males), nr. Requena, Jenaro Herrera, $4.900^{\circ} \mathrm{S}, 73.6303^{\circ} \mathrm{W}, 1$ August 2011 (G. Lamarre) (NMNH: ADP135885, male); Madre de Dios, Reserva Nacional Tambopata, 30 $\mathrm{km}$ (air) SW, Pto. Maldonado, Explorer's Inn, $12.8364^{\circ} \mathrm{S}, 69.2936^{\circ} \mathrm{W}, 209 \mathrm{~m}, 11-15$ November 1979 (JB Heppner, et al.)(NMNH: ADP007286, female), 28 February 1984 (TL Erwin, et al.)(NMNH: ADP007269, male, ADP007270, female), 9 March 1984 (TL Erwin, et al.)(NMNH: ADP007271, male), 4 May 1984 (TL Erwin, et al.) (NMNH: ADP007265, female), 10 May 1984 (TL Erwin, et al.)(NMNH: ADP007248, male), 6 September 1984 (TL Erwin, et al.)(NMNH: ADP007267, female), 10 September 1984 (TL Erwin, et al.)(NMNH: ADP007249, female), Manu Reserved Zone, Río Manu, BIOLAT Biological Station, Pakitza, 11.9446 ${ }^{\circ}$, $71.2831^{\circ} \mathrm{W}, 356 \mathrm{~m}, 7$ September 1988 (TL Erwin (NMNH: ADP007488, male), 2 September 1989 (TL Erwin, BD Farrell)(NMNH: ADP007487, male), 18 September 1988 (TL Erwin, BD Farrell)(NMNH: ADP007489, male), 19-23 September 1989 (N Adams)(NMNH: ADP007508, female), 22 September 1989 (TL Erwin (NMNH: ADP007446, female), 11 October 1989 (TL Erwin, GP Servat)(NMNH: ADP007485, female), 20 September 1991 (TL Erwin)(NMNH: ADP007403, male, ADP007418, female), 21 September 1991 (TL Erwin, et al.)(NMNH: ADP007464, female), 22 September 1991 (TL Erwin (NMNH: ADP007463, ADP007469, males), 26 September 1991 (TL Erwin (NMNH: ADP007381, ADP007335, males), 28 September 1991 (TL Erwin, MG Pogue)(NMNH: ADP007422, male), 30 September 1991 (TL Erwin (NMNH: ADP007424, ADP007309, ADP007330, ADP007396, ADP007400, ADP007425, males), 2 October 1991 (TL Erwin, MG Pogue)(NMNH: ADP007397, female), 4 October 1991 (TLErwin, MG Pogue)(NMNH: ADP007462, male), 16 October 1991 (TL Erwin, MG Pogue)(NMNH: ADP007419, ADP007420, males), 13-18 February 1992 (B Brown, D Feener)(NMNH: ADP007510, female), 4-9 March 1992, (B Brown, D Feener)(NMNH: ADP007509, female), 22 June 1993 (TL Erwin, F Pfuno S)(NMNH: ADP007465, ADP007468, males), 23 June 1993 (TL Erwin, F. Pfuno S)(NMNH: ADP007491, male, ADP007490, ADP007506, ADP007467, females). Suriname, Brokopondo, Phedra, $5.232^{\circ} \mathrm{N}, 55.050^{\circ} \mathrm{W}, 8 \mathrm{~m}$, 1-7 November 1964 (DC Geijskes)(NBCL: ADP005804, male),

Geographic distribution. (Fig. 11). This species is currently known from the type locality in the Amazonian lowlands near Tefé, Brazil, and in Brazil - Amazonas, Pará, Goias; Colombia; Ecuador; French Guiana; Perú; Suriname.

Notes. Reichardt $(1971,1973)$ reported the following additional specimens that we did not see: Brazil - Amazonas: Manaus (13 exs. MZSP), Maturacá, alto Rio Cauaburi (2 exs. MZSP), São Paulo de Olivença (1 ex. MZSP), Tefé (2 exs. MNHP), Titirico (1 ex. FAUCV); Pará: Belém (1 ex. MNHP, 1 ex. MZSP), Icoraci (1 ex. MNHP). Goias: Jatar (1 ex. MNHP), Rio Verde (1 ex. MNHP). Perú - Loreto: Chambiruyaca, near Yurimaguas (1 ex. MNHP), Pebas (2 exs. MNHP). 


\section{Hyboptera biolat Erwin \& Henry, sp. n.}

http://zoobank.org/896D1B12-7939-4277-8C55-A975042F72D9

Biolat humps-backed beetle

Figs 1B, 5B, 11

Holotype. Male. Perú, Madre de Dios, Manu Reserved Zone, Río Manu, BIOLAT Biological Station, Pakitza, $11.9446^{\circ} \mathrm{S}, 71.2831^{\circ} \mathrm{W}, 356 \mathrm{~m}, 20$ September 1991 (TL Erwin)(NMNH: ADP007447).

Derivation of specific epithet. The specific epithet, biolat, is used as a noun in apposition based on the acronym of the Smithsonian Institution's past Program "Biodiversity in Latin America" (BIOLAT) which sought to field-train young Latin American biology students in biodiversity techniques and did so for over 200 of them between 1987 and 1991 in Perú and Bolivia. These beetles were collected under the auspices of the BIOLAT Program.

Proposed English vernacular name. Biolat humps-backed beetle.

Diagnosis. With the attributes of the genus and angulicollis species group as described above and adults with patches of bright metallic green para-medially on pronotum, elytra entirely with metallic green patches, and venter with meso- and metathorax mostly infuscated.

Description. (Fig. 1B, 5B). Size: See Appendix 1. Length (SBL) short for genus, $\mathrm{ABL}=3.64-4.17 \mathrm{~mm}, \mathrm{SBL}=3.16-3.74 \mathrm{~mm}, \mathrm{TW}=1.68-2.33 \mathrm{~mm}$.

Color: See diagnosis, above.

Luster: Metallic highlights, partially iridescent.

Microsculpture: Mostly isodiametric or slightly stretched, shallowly impressed, cells somewhat more stretched around elytral tubercles.

Head: Rugae moderately coarse, mostly transverse. Eye very large, sub-hemispheric, evenly rounded anteriorly, subtly more prolonged posteriorly. Antenna short, barely reaching humerus. Labrum rectangulate, shallowly bilobed, anterior margin slightly emarginate. Neck finely and transversely rugose.

Prothorax: Pronotum moderately broad, disc centrally planar with dense transverse rugae. Lateral margins moderately explanate and obtusely angulate medially then straight to narrowly obtuse hind angle, base medially produced and rounded posteriorly.

Pterothorax: Normal for Agrina, fully winged. Elytron interval 3 with 7(8), and interval 5 with 5 (4) discal unisetiferous tubercles, other intervals moderately convex, side margin moderately explanate laterally only at middle third. Elytron broad and moderately short, moderately narrower than the pronotum at the broadest part, apex truncate, slightly rounded with distal corner broadly and obtusely rounded, disc not significantly convex, basal third slightly depressed. All interneurs well-impressed.

Legs: Femur dorso-ventrally moderately depressed, tibia coequal in length, more depressed; tarsus less than half the length of the tibia, fourth tarsomere markedly bilobed and with tarsal pad of setae. 
Abdomen: Sparsely setiferous; normal ambulatory setae on sterna 3-5; female with two pairs of ambulatory setae on sternum 6 , medial pair of setae less than the length of lateral pair; males with only the outer pair of longer setae on sternum 6 .

Male genitalia: Phallus (Fig. 5B) with ostium of $1 / 5$ its length, catopic, apex moderately long, narrowly rounded, narrow in dorsal aspect; endophallus with flagellum (apex extruded in illustration), flagellum not barbed. Parameres asymmetric, right very small, left larger.

Female genitalia: Not investigated, likely similar to that of H. lucida (Fig. 8).

Dispersal potential. These beetles are macropterous and probably capable of flight. They are moderately swift and agile runners. Specimens have been acquired using insecticidal fogging methods.

Way of life. Adults are common in the lowlands (356 m.a.s.l.) and appear to be generalists in a variety of rainforest biotopes including terra firme and upper floodplain forests. In these forests, they are commonly found in suspended dry leaves in Guadua weberbaueri Pilg. bamboo patches and among Astrocaryum chambira Burret palm dry leaf-skirts. Adults have been obtained in September-October; hence, they are active in the late dry and early rainy seasons.

Other specimens examined. Perú, Madre de Dios, Manu Reserved Zone, Río Manu, BIOLAT Biological Station, Pakitza, $11.9446^{\circ} \mathrm{S}, 71.2831^{\circ} \mathrm{W}, 356 \mathrm{~m}, 4$ October 1989 (TL Erwin)(NMNH, ADP007486, male paratype), 10 October 1991 (TL Erwin, MG Pogue)(NMNH: ADP007398, female paratype), 11 October 1991 (TL Erwin, MG Pogue)(NMNH: ADP007353, ADP007354, ADP007356, ADP007443, male paratypes, ADP007355 ADP007399, female paratypes), 14 October 1991 (TL Erwin, MG Pogue)(NMNH: ADP007336, male paratype), 16 October 1991 (TL Erwin)(NMNH: ADP007331, male paratype), 23 September 1991 (TL Erwin) (NMNH: ADP007466, male paratype), 28 September 1991 (TL Erwin, MG Pogue) (NMNH: ADP007315, male paratype), 9 October 1991 (TL Erwin, MG Pogue) (NMNH: ADP007311, male paratype).

Geographic distribution (Fig. 11). This species is currently known only from the type locality in Perú in the Río Manu watershed at the Pakitza Vigilante Post where the BIOLAT Biological Station operated from 1987 to 1992.

Notes. The holotype will be deposited in UNMSM and is currently held in trust until the completion of studies at NMNH. Specimen ADP007443 is missing its fore body.

\section{Hyboptera vestiverdis Henry \& Erwin, sp. n.}

http://zoobank.org/54DF5437-5E03-4BA5-AE7F-8352ED2FDA11

Leprechaun humps-backed beetle

Figs $2 \mathrm{~A}, 5 \mathrm{C}, 11$

Holotype. Male. Perú, Madre de Dios, Manu Reserved Zone, Río Manu, BIOLAT Biological Station, Pakitza, $11.9446^{\circ} \mathrm{S}, 71.2831^{\circ} \mathrm{W}, 356 \mathrm{~m}, 16$ October 1991 (TL Erwin, MG Pogue)(NMNH: ADP007421). 
Derivation of specific epithet. The species epithet "vestiverdis" is a Latinized feminine noun meaning green vest, referring to the two lobes of color on the pronotal disc on individuals of this species with central line of pale color bisecting the pattern and appearing as an open vest.

Proposed English vernacular name. Leprechaun humps-backed beetle.

Diagnosis. With the attributes of the genus and angulicollis species group as described above and adults with only dark slightly-metallic markings on the pronotal disc, elytra olivaceous, markedly shiny, some individuals with hint of metallic green near basal margin, narrow, with slightly arcuate lateral margin.

Description. (Figs 2A, 5C) Size: See Appendix 1. Length (SBL) short for genus, $\mathrm{ABL}=3.46-4.62 \mathrm{~mm}, \mathrm{SBL}=3.14-3.99 \mathrm{~mm}, \mathrm{TW}=1.83-2.55 \mathrm{~mm}$.

Color: See diagnosis, above.

Luster: Very shiny elytra, not iridescent.

Microsculpture: Mostly isodiametric and slightly stretched, shallowly impressed, cells somewhat more stretched around elytral tubercles.

Head: Rugae moderately coarse, mostly transverse, somewhat curved on occiput. Eye very large, nearly perfectly hemispheric, evenly rounded. Antenna short, barely reaching humerus. Labrum rectangulate, shallowly bilobed, anterior margin slightly emarginate. Neck finely transversely rugose.

Prothorax: Pronotum markedly broad, disc planar, with dense transverse rugae. Lateral margins broadly explanate and obtusely angulate medially, then markedly arcuate to obtuse hind angle, base medially produced and rounded posteriorly.

Pterothorax: Normal for Agrina, fully winged. Elytron intervals 3 with 7 and 5 with 5(6) elongate unisetiferous tubercles, other intervals moderately convex, side margin broadly explanate laterally only at middle third. Elytron broad and short, slightly narrower than the pronotum at the broadest part, apex truncate, slightly rounded with distal corner broadly and obtusely rounded, disc not significantly convex, basal third slightly depressed. All interneurs well-impressed.

Legs: Femur dorso-ventrally moderately depressed, tibia coequal in length, more depressed; tarsus less than half the length of the tibia, fourth tarsomere markedly bilobed and with tarsal pad of setae.

Abdomen: Sparsely setiferous; normal ambulatory setae on sterna 3-5; female unknown; males with only the lateral pair of longer setae on sternum 6 .

Male genitalia: Phallus (Fig. 5C) with ostium of 1/5 its length, catopic, apex moderately long, narrowly rounded; endophallus with flagellum (mid-part obvious in illustration), flagellum not barbed. Parameres asymmetric, right very small, left larger.

Female genitalia: Not investigated, likely similar to that of $H$. lucida (Fig. 8).

Dispersal potential. These beetles are macropterous and capable of flight. They are moderately swift and agile runners. Specimens have been acquired in Malaise traps and using insecticidal fogging methods.

Way of life. Adults are common in the lowlands (116 to 356 m.a.s.l.) and appear to be generalists in a variety of rainforest biotopes including terra firme and várzea. In these forests, they are commonly found in big trees with vines and epiphytes, in 

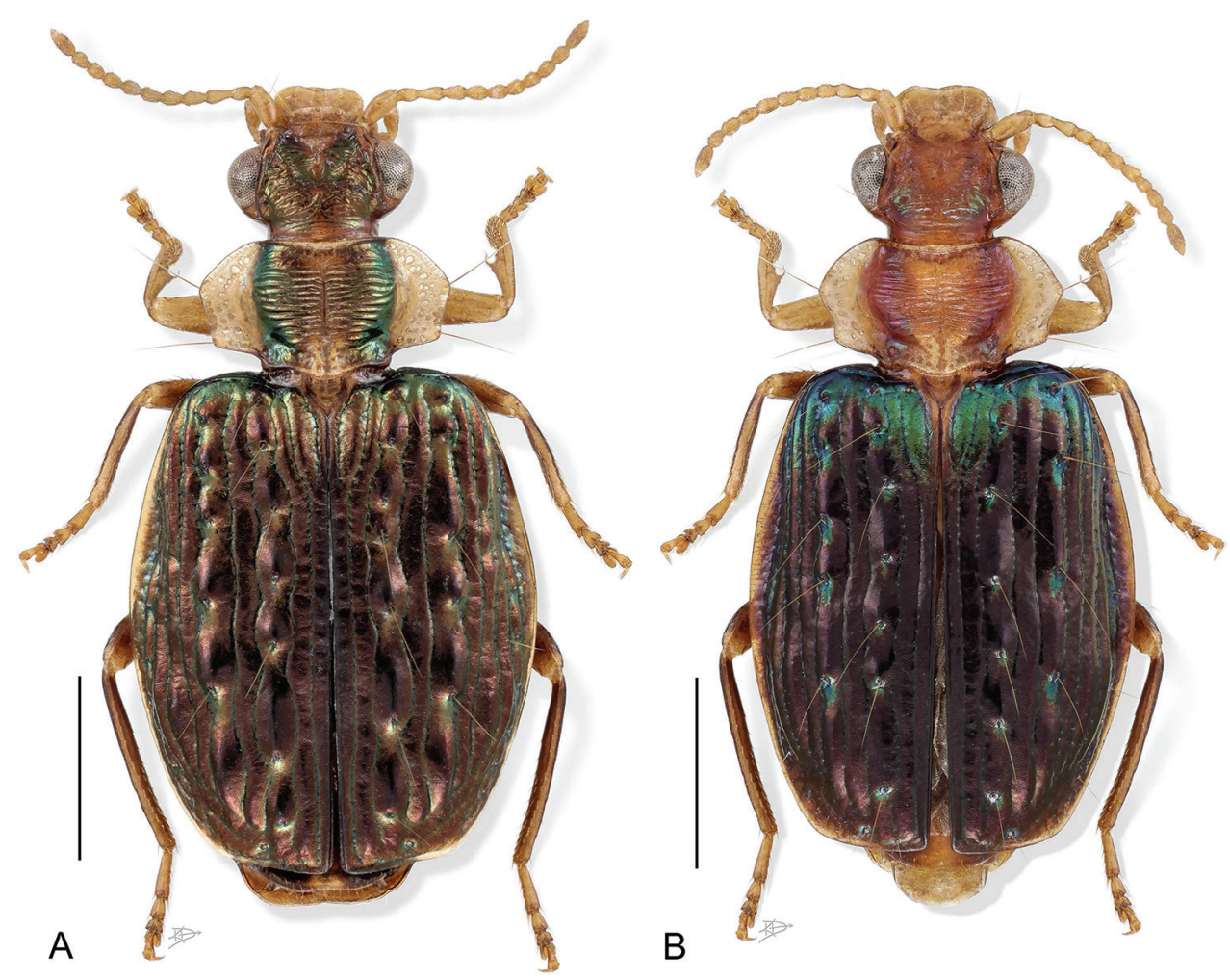

Figure 2. Digital Photo-illustration. Habitus, dorsal aspect. A Hyboptera vestiverdis Henry \& Erwin, sp. n., female, ADP007334 B Hyboptera scheelea Erwin \& Henry, sp. n., female, ADP007575.

suspended dry leaves, in dry Scheelea sp. and Astrocaryum chambira Burret palm frond skirts, in dry leaves scattered in vine tangles, and in dry leaves of the bamboo Guadua weberbaueri Pilg. Individuals can be found in January-February, May-July, and September-October, in both the rainy and dry seasons. Member of this species have been recorded from the canopy of the following tree species using insecticidal fogging techniques: Grias neuberthii; Eschweilera coriacea cf.; Icicopsis sp. ; Pentaplaris huaoranica sp. nov. Rhodostemonodaphne kunthiana cf.; Nectandra sp.; Iriartea deltoidea; Protium sagotianum cf.; Talisia bitter; Mouriri guapira; Eriotheca globosa cf.; Sloane sp., Matisia malacocalyx cf.; Tabebuia moby; Pourouma bicolor ssp. bicolor cf.; Alchornea triplinervia cf.?; Naucleopsis krukovii cf.; Hyeronima oblonga; Brownea grandiceps cf.; Virola decorticans cf.; Pouteria cuspidata ssp. robusta cf.; Duguetia surinamensis cf.; Trichilia rubra cf.; Coccoloba densifrons cf. Eschweilera coriacea cf.; Virola obovata; Brosimum utile ssp. ovatifolium cf.; Pseudolmedia laevis; Patinoa paraensis/sphaerocarpa cf.; Matisia bracteolosa stellate; Simaba guianensis cf.; Trichilia elsae cf.; Icicopsis sp. nov.

Other specimens examined. Ecuador, Orellana, Reserva Ethnica Huaorani, 39 km S Pompeya, Estación Científica Yasuní - Onkone Gare Camp, Erwin Piraña Plot, transect 2 , station $10,0.6581^{\circ} \mathrm{S}, 76.4513^{\circ} \mathrm{W}, 220-250 \mathrm{~m}, 4$ October 1995 (TL 
Erwin, et al.)(NMNH: ADP135922, female paratype), Erwin Pirańa Plot, transect 3, station $1,0.6575^{\circ} \mathrm{S}, 76.4505^{\circ} \mathrm{W}, 220-250 \mathrm{~m}, 1$ July 1995 (TL Erwin, et al.) (NMNH: ADP135976, male paratype), Erwin Pirańa Plot, transect 3, station 9, $0.6575^{\circ} \mathrm{S}$, $76.4505^{\circ} \mathrm{W}, 220-250 \mathrm{~m}, 21$ January 2006 (TL Erwin, et al.)(NMNH: ADP133860, female paratype), Erwin Piraña Plot, transect 4, station 3, $0.6570^{\circ} \mathrm{S}, 76.4498^{\circ} \mathrm{W}$, 220-250m, 11 February 1995 (TL Erwin, et al.)(NMNH: ADP137321, male paratype), Erwin Piraña Plot, transect 4, station 3, $0.6570^{\circ} \mathrm{S}, 76.4498^{\circ} \mathrm{W}, 220-250 \mathrm{~m}$, 11 February 1995 (TL Erwin, et al.)(NMNH: ADP135848, female paratype), Erwin Piraña Plot, transect 4, station 3, $0.6570^{\circ} \mathrm{S}, 76.4498^{\circ} \mathrm{W}, 220-250 \mathrm{~m}, 11$ February 1995 (TL Erwin, et al.)(NMNH: ADP135908, female paratype), Erwin Piraña Plot, transect 4 , station $3,0.6570^{\circ} \mathrm{S}, 76.4498^{\circ} \mathrm{W}, 220-250 \mathrm{~m}, 16$ January 1994 (TL Erwin, et al.)(NMNH: ADP135934, female paratype), Erwin Pirańa Plot, transect 5, station $10,0.6566^{\circ} \mathrm{S}, 76.4490^{\circ} \mathrm{W}, 220-250 \mathrm{~m}, 6$ October 1996 (TL Erwin, et al.)(NMNH: ADP135896, female paratype), Erwin Piraña Plot, transect 5, station 5, $0.6575^{\circ} \mathrm{S}, 76.4505^{\circ} \mathrm{W}, 220-250 \mathrm{~m}, 6$ January 1995 (TL Erwin, et al.) (NMNH: ADP135962, female paratype), Erwin Piraña Plot, transect 6, station 10, 0.6561 ${ }^{\circ} \mathrm{S}$, $76.4483^{\circ} \mathrm{W}, 220-250 \mathrm{~m}, 2$ October 1996 (TL Erwin, et al.)(NMNH: ADP135932, female paratype), Erwin Pirańa Plot, transect 6, station 3, $0.6561^{\circ} \mathrm{S}, 76.4481^{\circ} \mathrm{W}$, 220-250m, 6 October 1995 (TL Erwin, et al.)(NMNH: ADP137333, male paratype), Erwin Pirańa Plot, transect 8 , station $10,0.6551^{\circ} \mathrm{S}, 76.4403^{\circ} \mathrm{W}, 220-250 \mathrm{~m}$, 3 October 1996 (TL Erwin, et al.)(NMNH: ADP137371, male paratype), Erwin Piraña Plot, transect 8 , station $8,0.6551^{\circ} \mathrm{S}, 76.4403^{\circ} \mathrm{W}, 220-250 \mathrm{~m}, 8$ February 1996 (TL Erwin, et al.)(NMNH: ADP137381, male paratype), Erwin Piraña Plot, transect 9, station $7,0.6545^{\circ} \mathrm{S}, 76.4460^{\circ} \mathrm{W}, 220-250 \mathrm{~m}, 8$ October 1995 (TL Erwin, et al.)(NMNH: ADP135986, female paratype); Yasuni National Park (edge), $95.43 \mathrm{~km}$ E (heading $101.46^{\circ}$ ) Coca, Tiputini Biodiversity Station, Erwin Harpia Plot, transect 1 , station $6,0.6342^{\circ} \mathrm{S}, 76.1443^{\circ} \mathrm{W}, 214 \mathrm{~m}, 2$ October 2000 (TL Erwin, et al.)(NMNH: ADP139098, male paratype), Erwin Harpia Plot, transect 5, station $8,0.6309^{\circ} \mathrm{S}, 76.1443^{\circ} \mathrm{W}, 220-250 \mathrm{~m}, 1$ July 1998 (TL Erwin, et al.)(NMNH: ADP135870, female paratype), Erwin Harpia Plot, transect 7, station 6, $0.6287^{\circ} \mathrm{S}$, $76.1443^{\circ} \mathrm{W}, 203 \mathrm{~m}, 22$ October 1998 (TL Erwin, et al.)(NMNH: ADP135855, male paratype), Erwin Harpia Plot, transect 7, station 9, 0.6287 ${ }^{\circ}$, 76.1443 ${ }^{\circ} \mathrm{W}, 203 \mathrm{~m}, 29$ September 2000 (TL Erwin, et al.)(NMNH: ADP139138, male paratype); SucumBíos, Río Napo, Sacha Lodge, Pilchicocha, $0.472^{\circ} \mathrm{S}, 76.459^{\circ} \mathrm{W}, 228 \mathrm{~m}, 12-22$ February 1994 (P Hibbs)(SEMC: ADP006133, male paratype). Perú, Loreto, PacayaSamiria National Reserve, Rio Samiria, Pithecia, 5.2116 S, 74.6991 W, 116m, 9 May 1990 (TL Erwin, GP Servat, et al.)(NMNH: ADP007293, male paratype), Boca del Ingles Camp, Hamburgo, 5.6951으, 75.2243W, 150m, 10 May 1990 (TL Erwin, et al.)(NMNH: ADP007292, male paratype, ADP007574, female paratype); MADRE DE Dios, Manu Reserved Zone, Río Manu, BIOLAT Biological Station, Pakitza, $11.9446^{\circ} \mathrm{S}, 71.2831^{\circ} \mathrm{W}, 356 \mathrm{~m}, 11$ October 1991 (TL Erwin, MG Pogue)(NMNH: ADP005780, male paratype), 11 October 1991 (TL Erwin)(NMNH: ADP007314, ADP007359, ADP007374, ADP007375, ADP007376, ADP007377, ADP007378, 
ADP007379, ADP007380, male paratypes, ADP007358, ADP007357, female paratypes), 14 October 1991 (TL Erwin, MG Pogue, NMNH: ADP007333, ADP007334, ADP007337, ADP007352, male paratypes), 16 October 1991 (TL Erwin)(NMNH: ADP007332, male paratype), (TL Erwin, MG Pogue)(NMNH: ADP007402, male paratype, ADP007313, ADP007401, female paratypes), 22 June 1993 (TL Erwin, F Pfuno S)(NMNH: ADP007484, female paratype), 23 June 1993 (TL Erwin, F Pfuno S)(NMNH: ADP007507, female paratype), 9 September 1988 (TL Erwin) (NMNH: ADP007445, male paratype), 30 September 1991 (TL Erwin, MG Pogue) (NMNH: ADP007423, male paratype), 9 October 1991 (TL Erwin, MG Pogue) (NMNH: ADP007442, male paratype, ADP007308, ADP007310, ADP007312, ADP007440, ADP007441, female paratypes); Reserva Nacional Tambopata, 30km (air) SW Pto. Maldonado, $12.8364^{\circ} \mathrm{S}, 69.2936^{\circ} \mathrm{W}, 209 \mathrm{~m}, 28$ February 1984 (TL Erwin, et al.)(NMNH: ADP007268, male paratype), 4 May 1984 (TL Erwin, et al.) (NMNH: ADP007266, male paratype).

Geographic distribution (Fig. 11). This species is currently known from the western Amazon Basin in lowland Ecuador and Perú.

Notes. The holotype will be deposited in UNMSM and is currently held in trust until the completion of studies at NMNH.

\section{Hyboptera scheelea Erwin \& Henry, sp. n.}

http://zoobank.org/6F9E1741-7310-4CB0-843F-0771EE17059F

Palm-frond humps-backed beetle

Figs 2B, 11

Holotype. Female. Perú, Loreto, Pacaya-Samiria National Reserve, Río Samiria (South Branch), Camp Terry, 5.6951 ${ }^{\circ} \mathrm{S}, 75.2243^{\circ} \mathrm{W}, 129 \mathrm{~m}, 14$ May 1990 (TL Erwin) (NMNH: ADP007575).

Derivation of specific epithet. The species epithet "scheelea" is used as a noun in apposition which is based on the genus of palm upon which the holotype was found.

Proposed English vernacular name. Palm-frond humps-backed beetle.

Diagnosis. With the attributes of the genus and angulicollis species group as described above and adults without pronotal markings, pronotal surface rufotestaceous, elytron blackish-blue with metallic green highlights across the humeri and green points at some larger tubercles with lateral margin narrowly testaceous to latero-apical corner but not reaching sutural apex. Apical abdominal tergite mostly infuscated with narrow median testaceous stripe. Size smaller than $H$. shasta adults.

Description. (Fig. 2B). Size: See Appendix 1. Length (SBL) short for genus, ABL $=4.54 \mathrm{~mm}, \mathrm{SBL}=3.68 \mathrm{~mm}, \mathrm{TW}=2.10 \mathrm{~mm}$.

Color: See diagnosis, above.

Luster: Metallic highlights, partially iridescent.

Microsculpture: Mostly isodiametric, shallowly impressed, cells somewhat stretched around elytral tubercles. 
Head: Rugae moderately coarse, mostly transverse. Eye very large, sub-hemispheric, evenly rounded anteriorly, subtly more prolonged posteriorly. Antenna short, barely reaching humerus. Labrum rectangulate, shallowly bilobed, anterior margin slightly emarginate. Neck finely and transversely rugose.

Prothorax: Pronotum markedly broad, disc centrally depressed with dense transverse rugae. Lateral margins broadly explanate and obtusely angulate medially then moderately arcuate to obtuse hind angle, base medially produced and rounded posteriorly.

Pterothorax: Normal for Agrina, fully winged. Elytron intervals 3 and 5 each with (4) 5 discal unisetiferous tubercles, interval 3 with one such tubercle near apex, other intervals moderately convex, side margin broadly explanate laterally only at middle third. Elytron broad and short, slightly narrower than the pronotum at the broadest part, apex truncate, slightly rounded with distal corner broadly and obtusely rounded, disc not significantly convex, basal third slightly depressed. All interneurs well-impressed.

Legs: Femur dorso-ventrally moderately depressed, tibia coequal in length, more depressed; tarsus less than half the length of the tibia, fourth tarsomere markedly bilobed and with tarsal pad of setae.

Abdomen: Sparsely setiferous; normal ambulatory setae on sterna 3-5; female with two pairs of ambulatory setae on sternum 6, medial pair of setae less than the length of lateral pair; males unknown.

Male genitalia: Unknown.

Female genitalia: Not investigated, likely similar to that of H. lucida (Fig. 8).

Dispersal potential. These beetles are macropterous and probably capable of flight. They are moderately swift and agile runners. The holotype was acquired by insecticidal fogging of a Sheelea palm.

Way of life. The single known adult was found in May in lowlands (129 m.a.s.l.) in the secondary floodplain of igapó forests.

Other specimens examined. None.

Geographic distribution (Fig. 11). This species is currently known only from the type locality in the lowland of Amazonian Perú.

Notes. The holotype will be deposited in UNMSM and is currently held in trust until the completion of studies at NMNH.

\section{Hyboptera shasta Erwin, sp. n.}

http://zoobank.org/F618555F-D7D8-43BA-B762-2E362948D342

Shasta's humps-backed beetle

Figs 3A, 11

Holotype. Male. Brazil, Amazonas, north of Manaus on Amazonas 010 at Km 26, Reserva Ducke, 2.918 S, 59.971 ${ }^{\circ} \mathrm{W}, 70 \mathrm{~m}, 4$ July 1978 (J Arias)(NMNH: ADP135875).

Derivation of specific epithet. The species epithet "shasta" is an eponym based on the first name of the coauthor of this paper and former Intern in the laboratory of the senior author of this paper at the Smithsonian Institution. 

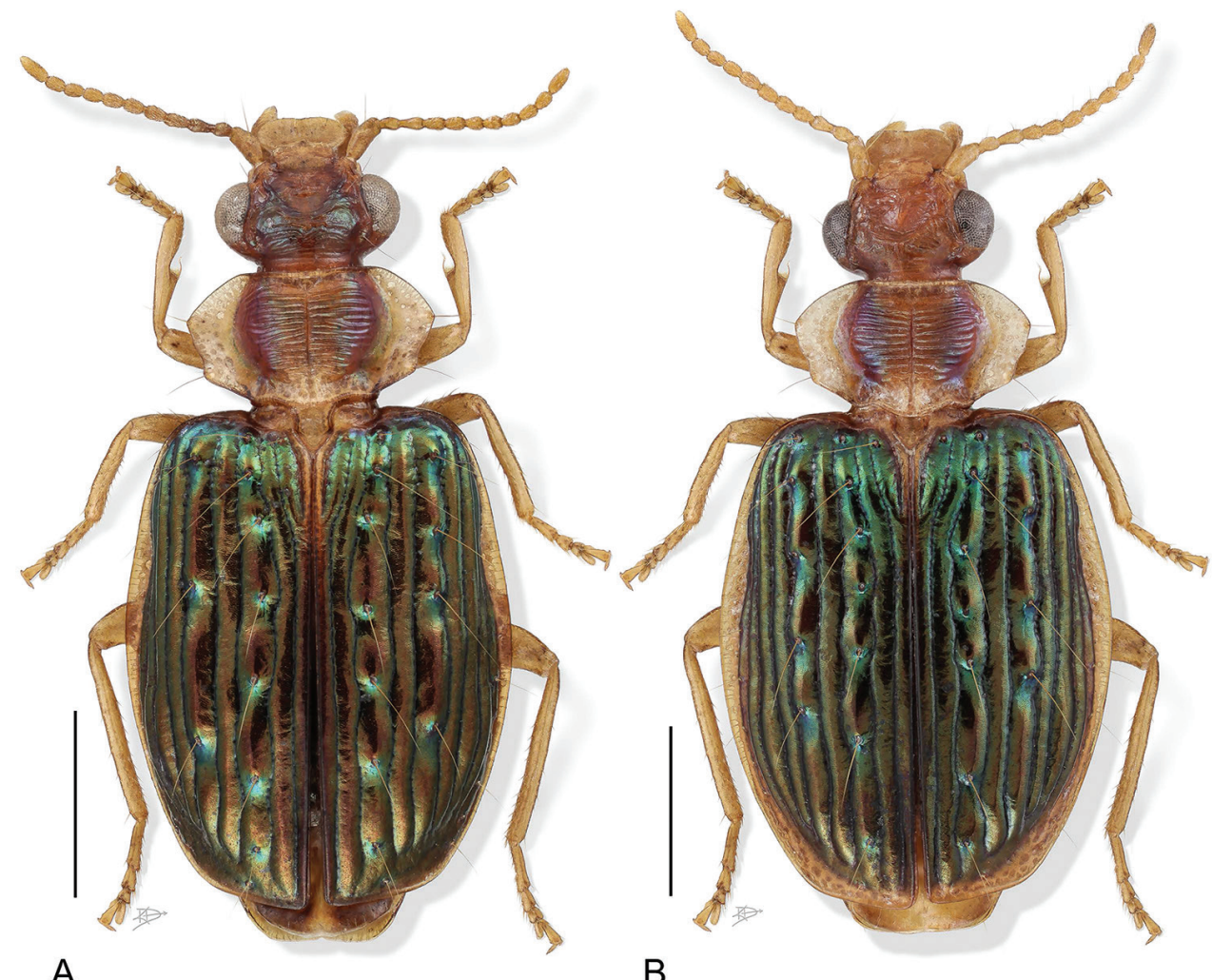

Figure 3. Digital Photo-illustration. Habitus, dorsal aspect. A Hyboptera shasta Erwin, sp. n., male, ADP135875 B Hyboptera tepui Erwin \& Henry, sp. n., male, ADP007676.

Proposed English vernacular name. Shasta’s humps-backed beetle.

Diagnosis. With the attributes of the genus and angulicollis species group as described above and adults without pronotal markings, pronotal surface rufotestaceous, elytron brilliant metallic green throughout with lateral margin testaceous to latero-apical corner, not quite reaching sutural apex; apical abdominal tergite mostly infuscated with narrow median testaceous stripe; size larger than $H$. scheelea adults.

Description. (Fig. 3A). Size: See Appendix 1. Length (SBL) medium for genus, $\mathrm{ABL}=4.82 \mathrm{~mm}, \mathrm{SBL}=3.86 \mathrm{~mm}, \mathrm{TW}=2.17 \mathrm{~mm}$.

Color: See diagnosis, above.

Luster: Very shiny elytra, substantially iridescent.

Microsculpture: Mostly isodiametric and slightly stretched, shallowly impressed, cells somewhat more stretched around elytral tubercles.

Head: Rugae moderately coarse, mostly transverse. Eye very large, nearly perfectly hemispheric, evenly rounded. Antenna short, barely reaching humerus. Labrum rectangulate, shallowly bilobed, anterior margin slightly emarginate. Neck finely transversely rugose. 
Prothorax: Pronotum markedly broad, disc not centrally depressed, with dense transverse rugae. Lateral margins broadly explanate and obtusely angulate medially, then moderately arcuate to obtuse hind angle, base medially produced and rounded posteriorly.

Pterothorax: Normal for Agrina, fully winged. Elytron intervals 3 with 7 and 5 with 3/4 elongate unisetiferous tubercles, other intervals moderately convex, side margin broadly explanate laterally only at middle third. Elytron broad and short, slightly narrower than the pronotum at the broadest part, apex truncate, slightly rounded with distal corner broadly and obtusely rounded, disc not significantly convex, basal third slightly depressed. All interneurs well-impressed.

Legs: Femur dorso-ventrally moderately depressed, tibia coequal in length, more depressed; tarsus less than half the length of the tibia, fourth tarsomere markedly bilobed and with tarsal pad of setae.

Abdomen: Sparsely setiferous; normal ambulatory setae on sterna 3-5; female unknown; males with only the lateral pair of longer setae on sternum 6 .

Male genitalia: Not investigated as only the holotype male is available, likely similar to that of $H$. angulicollis (Fig. 5A).

Female genitalia: Not investigated, likely similar to that of H. lucida (Fig. 8).

Dispersal potential. These beetles are macropterous and capable of flight. They are moderately swift and agile runners. The holotype was acquired in a C.D.C. light trap.

Way of life. The single known adult was found in July in lowlands (70 m.a.s.l.) in the terra firme forests.

Other specimens examined. None.

Geographic distribution (Fig. 11). This species is currently known only from the type locality in lowland Amazonian Brazil.

\section{Hyboptera tepui Erwin \& Henry, sp. n.}

http://zoobank.org/C3DF93E5-F8A5-452F-BDBD-ED2EF3D1F438

Tepui humps-backed beetle

Figs 3B, 11

Holotype. Female. Venezuela, Amazonas, Cerro de la Neblina, Río Baria Basecamp, $0.837^{\circ} \mathrm{N}, 66.162^{\circ} \mathrm{W}, 138 \mathrm{~m}, 20$ February 1985 (PJ Spangler, PM Spangler, et al.) (NMNH: ADP007576).

Derivation of specific epithet. The species epithet "tepui" is used as a noun in apposition and is based on the type of Venezuelan flat-topped upland near which the holotype was found.

Proposed English vernacular name. Tepui humps-backed beetle.

Diagnosis. With the attributes of the genus and angulicollis species group as described above and adults without pronotal markings, pronotal surface rufotestaceous. Elytron with lateral margin broadly testaceous from humerus to sutural apex; apical abdominal tergite testaceous with slight infuscation at extreme posterior-lateral corners.

Description. (Fig. 3B). Size: See Appendix 1. Length (SBL) long for genus, ABL $=5.16 \mathrm{~mm}, \mathrm{SBL}=4.29 \mathrm{~mm}, \mathrm{TW}=2.458 \mathrm{~mm}$. 
Color: See diagnosis, above.

Luster: Metallic green highlights, partially iridescent.

Microsculpture: Mostly irregular isodiametric, often stretched, shallowly impressed, cells especially stretched around elytral tubercles.

Head: Rugae moderately coarse, mostly transverse. Eye very large, sub-hemispheric, evenly rounded anteriorly, subtly more prolonged posteriorly. Antenna short, barely reaching humerus. Labrum rectangulate, shallowly bilobed, anterior margin slightly emarginate. Neck finely transversely rugose.

Prothorax: Pronotum markedly broad, disc with dense transverse rugae. Lateral margins broadly explanate and obtusely angulate medially then moderately arcuate to obtuse hind angle, base medially produced and rounded posteriorly.

Pterothorax: Normal for Agrina, fully winged. Elytron intervals 3 and 5 each with 6 discal unisetiferous tubercles, interval 3 with one subtle tubercle near apex, other intervals shallowly to moderately convex, side margin broadly explanate laterally only at middle third. Elytron broad and short, slightly narrower than the pronotum at the broadest part, apex truncate, slightly rounded with distal corner broadly and obtusely rounded, disc not significantly convex, basal third slightly depressed. All interneurs well-impressed.

Legs: Femur dorso-ventrally moderately depressed, tibia coequal in length, more depressed; tarsus less than half the length of the tibia, fourth tarsomere markedly bilobed and with tarsal pad of setae.Abdomen: Sparsely setiferous; normal ambulatory setae on sterna 3-5; female with two pairs of ambulatory setae on sternum 6 , medial pair of setae less than the length of lateral pair; males unknown.

Male genitalia: Unknown.

Female genitalia: Not investigated, likely similar to that of $H$. lucida (Fig. 8).

Dispersal potential. These beetles are macropterous and capable of flight. They are moderately swift and agile runners. The holotype was acquired at black light in a rainforest clearing.

Way of life. The single known adult was found in February in lowland (138 m.a.s.l.) terra firme forests.

Other specimens examined. None.

Geographic distribution (Fig. 11). This species is currently known only from the type locality in lowland Venezuela.

\section{Hyboptera tiputini Erwin \& Henry, sp. n.}

http://zoobank.org/04C99837-BEFF-4F3E-BE65-69CA3562A4A0

Tiputini humps-backed beetle

Figs 4A, 5D, 11

Holotype. Male. Ecuador, Orellana, Yasuni National Park (edge), $95.43 \mathrm{~km}$ E (heading $101.46^{\circ}$ ) Coca, Tiputini Biodiversity Station, Erwin Harpia Plot: transect 4, station $4,0.6316^{\circ} \mathrm{S}, 76.1443^{\circ} \mathrm{W}, 208 \mathrm{~m}, 8$ February 1999 (TL Erwin, et al.)(NMNH: ADP135781). 

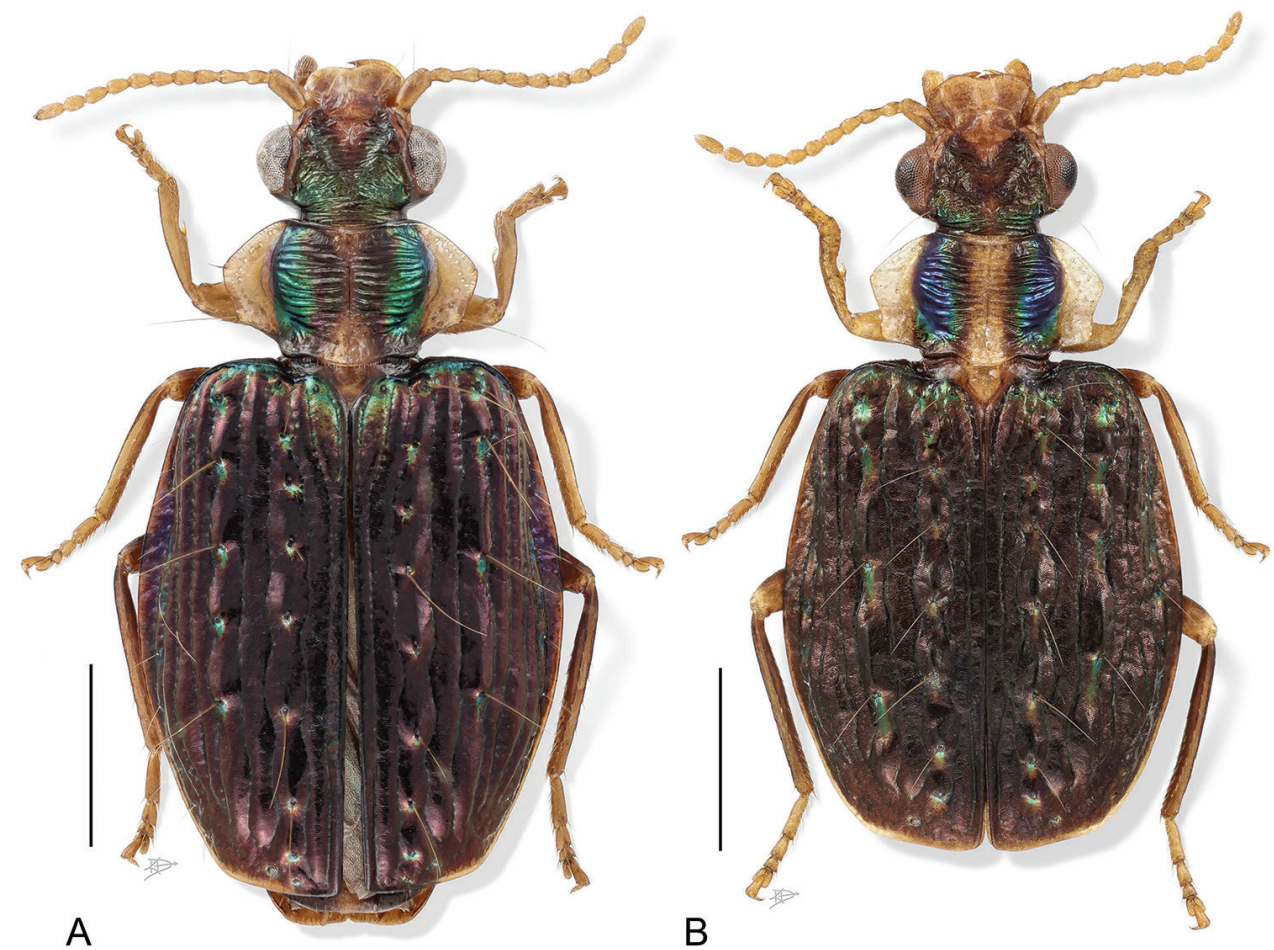

Figure 4. Digital Photo-illustration. Habitus, dorsal aspect. A Hyboptera tiputini Erwin \& Henry, sp. n., male, ADP135850 B Hyboptera viridivittis Chaudoir, male, ADP007599.

Derivation of specific epithet. The specific epithet, "tiputini" is used as a noun in apposition and is based on the Tiputini Biodiversity Station in the Yasuni area of eastern Ecuador in reference to one of the places in which members of this species are found.

Proposed English vernacular name. Tiputini humps-backed beetle.

Diagnosis. With the attributes of the genus and angulicollis species group as described above and adults with patches of bright metallic green para-medially on pronotum, elytra markedly shiny, violaceous, some individuals with hint of metallic green near basal margin, broad, with markedly arcuate lateral margin.

Description. (Figs 4A, 5D). Size: See Appendix 1. Length (SBL) long for genus, $\mathrm{ABL}=4.21-5.64 \mathrm{~mm}, \mathrm{SBL}=3.33-4.72 \mathrm{~mm}, \mathrm{TW}=1.868-2.92 \mathrm{~mm}$.

Color: See diagnosis, above.

Luster: Matte with a few green reflections, markedly iridescent.

Microsculpture: Mostly irregular isodiametric, often stretched, shallowly impressed, cells especially stretched around elytral tubercles.

Head: Rugae moderately coarse, transverse. Eye very large, sub-hemispheric, evenly rounded anteriorly, subtly more prolonged posteriorly. Antenna short, barely reaching humerus. Labrum rectangulate, shallowly bilobed, anterior margin slightly emarginate. Neck finely transversely rugose. 

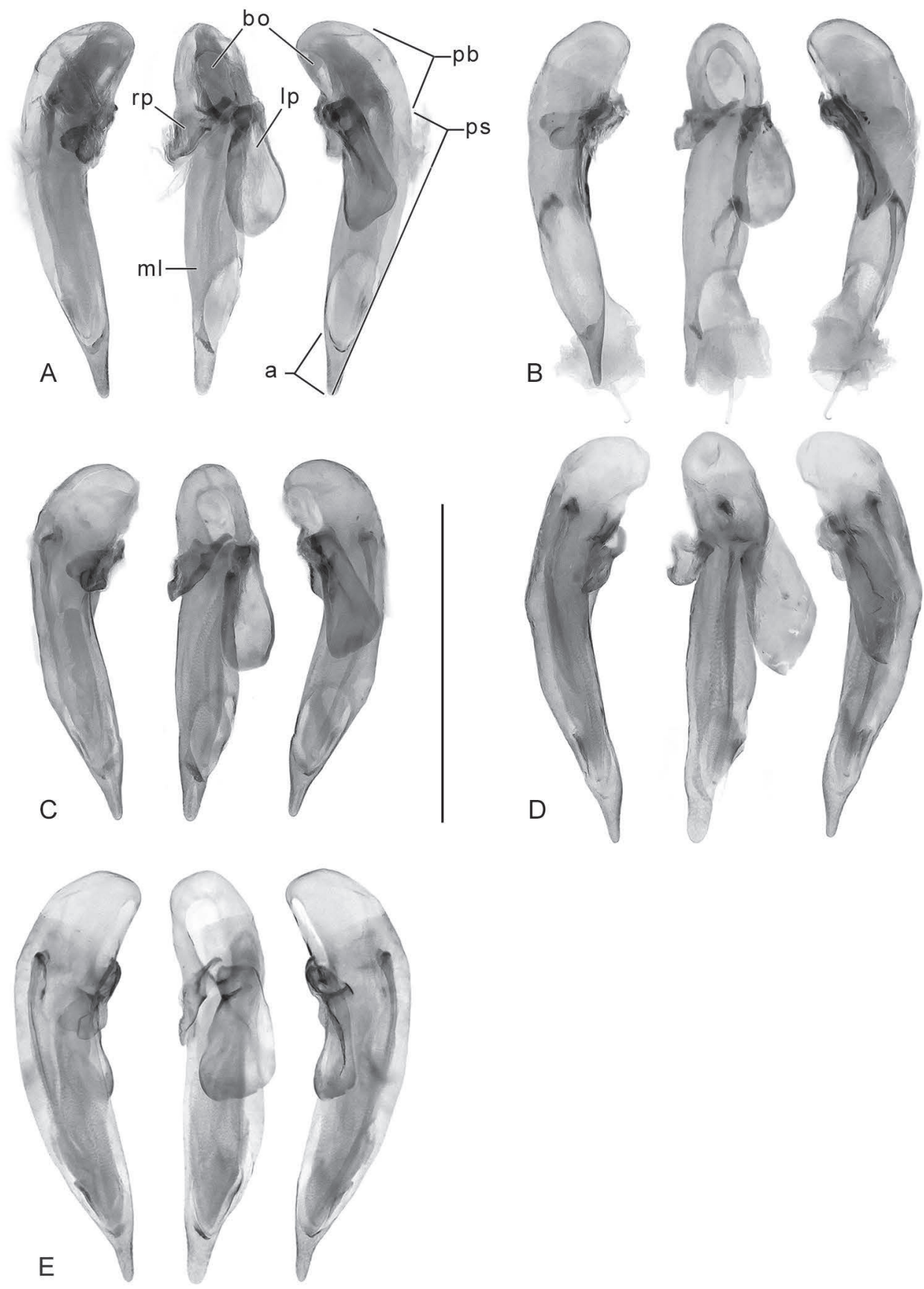

Figure 5. Digital Photo-illustration, male aedeagus in repose, dorsal, ventral, left lateral aspects. A Hyboptera angulicollis Chaudoir, ADP007330 B Hyboptera biolat Erwin \& Henry, sp. n., ADP007443 C Hyboptera vestiverdis Henry \& Erwin, sp. n., ADP014856 D Hyboptera tiputini Erwin \& Henry, sp. n., ADP135845 E Hyboptera viridivittis Chaudoir, ADP007601. Legend, lp, left paramere; rp, right paramere; pb, phallobase; bo, phallobase orifice; ps, phalloshaft; a, phalloapex. Scale line $=0.25 \mathrm{~mm}$. 
Prothorax: Pronotum markedly broad, disc medially shallowly depressed along midline, and with dense transverse rugae. Lateral margins broadly explanate and obtusely angulate medially then subtlely arcuate to obtuse hind angle, base medially produced and rounded posteriorly.

Pterothorax: Normal for Agrina, fully winged. Elytron interval 3 with 7(8) and interval 5 with 5 unisetiferous tubercles, other intervals shallowly to moderately convex, side margin broadly explanate laterally only at middle third. Elytron broad and short, slightly narrower than the pronotum at the broadest part, apex truncate, slightly rounded with distal corner broadly and obtusely rounded, disc not significantly convex, basal third slightly depressed. All interneurs well-impressed.

Legs: Femur dorso-ventrally moderately depressed, tibia coequal in length, more depressed; tarsus less than half the length of the tibia, fourth tarsomere markedly bilobed and with tarsal pad of setae.

Abdomen: Sparsely setiferous; normal ambulatory setae on sterna 3-5; female with two pairs of ambulatory setae on sternum 6 , medial pair of setae less than the length of lateral pair; males unknown.

Male genitalia: Phallus (Fig. 5D) with ostium of 1/5 its length, catopic, apex moderately long, narrowly rounded, broad in dorsal aspect; endophallus with flagellum (mid-part obvious in illustration), flagellum not barbed. Parameres asymmetric, right very small, left larger.

Female genitalia: Not investigated, likely similar to that of $H$. lucida (Fig. 8).

Dispersal potential. These beetles are macropterous and probably capable of flight. They are moderately swift and agile runners.

Way of life. Adults are common in the lowlands and lower midlands (20 to 900 $\mathrm{m}$ a.s.l.) and appear to be generalists in a variety of rainforest biotopes including terra firme, várzea, and igapó. In these forests, they are commonly found in big trees with vines and epiphytes, in suspended dry leaves, in dry Astrocaryum chambira Burret palm frond skirts. Individuals can be found in January-November, in both the rainy and dry seasons. Member of this species have been recorded from the canopy of the following tree species using insecticidal fogging techniques: Pseudolmedia laevis; Iriartea deltoidea; Castilla ulei cf.; Sorocea steinbachii cf.; Matisia malacocalyx cf. Swartzia león; Eschweilera coriacea cf.; Mouriri guapira; Trichilia solitudinis; Eriotheca globosa cf.; Sloanea 1; Vismia weedy; Tabebuia moby; Pourouma bicolor ssp. bicolor cf.; Alchornea triplinervia cf.?; Naucleopsis krukovii cf.; Hyeronima oblonga; Clarisia biflora; Pouteria baehniana cf.; Inga cuadra; Maquira calophylla; Brownea grandiceps cf.; Talisia bitter; Virola decorticans cf.; Pouteria cuspidata ssp. robusta cf.; Diospyros sericea; Guarea silvatica; Scheelea sp.

Other specimens examined. Colombia, Amazonas, PNN Amacayacu, Mocagua, $3.84^{\circ} \mathrm{S}, 70.22^{\circ} \mathrm{W}, 76 \mathrm{~m}, 14-21$ August 2000 (A Parente)(IAvH: AvH-E-73764, ADP145179, female paratype), 12-19 March 2000 (A Parente)(IAvH: AvH-E-2971, ADP145201, female paratype), 19-26 June 2000 (A Parente)(IAvH: AvH-E-73760, ADP145205, ADP145213, female paratypes), 2-8 May 2000 (A Parente)(IAvH: AvH-E-73762, ADP145199, female paratype), 29 May - 6 June 2000 (A Parente) (IAvH: AvH-E-73759, ADP145207, female paratype), 3-9 April 2000 (A Parente) 
(IAvH: AvH-E-73758, ADP145185, female paratype); CHocó, Ensenada de Utría Cocalito, $6.046^{\circ} \mathrm{N}, 77.352^{\circ} \mathrm{W}, 20 \mathrm{~m}, 1$ August - 10 October 2000 (S Sarria)( IAvH: IAvH-E-10943, ADP145168, female paratype); Valle del Cauca, PPN Farallones de Cali, $3.527^{\circ} \mathrm{N}, 76.848^{\circ} \mathrm{W}, 650-900 \mathrm{~m}, 1$ August - 10 October 2000 (S Sarria)( IAvH: IAvH-E-3531, ADP145212, male paratype, IAvH-E-10942, ADP145208, IAvH-E-40743, ADP145216, female paratypes). Ecuador, Orellana, Reserva Ethnica Huaorani, 39 km S Pompeya, Estación Científica Yasuní - Onkone Gare Camp, $0.6551^{\circ} \mathrm{S}, 76.4403^{\circ} \mathrm{W}, 220-250 \mathrm{~m}, 4-14$ October 1995 (GE Ball, D.Shpeley)(UASM: ADP135771, male paratype), Erwin Piraña Plot: transect 6, station 9, $0.6561^{\circ} \mathrm{S}$, $76.4483^{\circ} \mathrm{W}, 220-250 \mathrm{~m}, 15$ January 1994 (TL Erwin, et al.)(NMNH: ADP138483, male paratype), Erwin Pirańa Plot: transect 2, station 8, 0.6581 ${ }^{\circ} \mathrm{S}, 76.4513^{\circ} \mathrm{W}, 220$ 250m, 20 June 1994 (TL Erwin, et al.)(NMNH: ADP135847, male paratype), Erwin Piraña Plot: transect 4, station 9, 0.6570 S, $76.4498^{\circ} \mathrm{W}, 220-250 \mathrm{~m}, 25$ June 1994 (TL Erwin, et al.)(NMNH: ADP135954, male paratype), Erwin Piraña Plot: transect 5, station 9, $0.6566^{\circ} \mathrm{S}, 76.4490^{\circ} \mathrm{W}, 220-250 \mathrm{~m}, 12$ February 1995 (TL Erwin, et al.)(NMNH: ADP137319, female paratype), Erwin Piraña Plot: transect 5, station $10,0.6566^{\circ} \mathrm{S}, 76.4490^{\circ} \mathrm{W}, 220-250 \mathrm{~m}, 6$ October 1995 (TL Erwin, et al.) (NMNH: ADP137341, male paratype, ADP137377, female paratype), Erwin Pirańa Plot: transect 10 , station $1,0.6540^{\circ} \mathrm{S}, 76.4453^{\circ} \mathrm{W}, 220-250 \mathrm{~m}, 8$ October 1995 (TL Erwin, et al.)(NMNH: ADP135950, female paratype), Erwin Pirańa Plot: transect 6, station $8,0.6561^{\circ} \mathrm{S}, 76.4483^{\circ} \mathrm{W}, 220-250 \mathrm{~m}, 7$ February 1996 (TL Erwin, et al.)(NMNH: ADP137389, female paratype), Erwin Piraña Plot: transect 4, station $4,0.6570^{\circ} \mathrm{S}, 76.4498^{\circ} \mathrm{W}, 220-250 \mathrm{~m}, 21$ June 1996 (TL Erwin, et al.)(NMNH: ADP137323, female paratype), Erwin Pirańa Plot: transect 5, station 5, $0.6566^{\circ} \mathrm{S}$, 76.4490 W, 220-250m, 22 June 1996 (TL Erwin, et al.)(NMNH: ADP137738, male paratype, ADP137349, female paratype), Erwin Piraña Plot: transect 1, station 9, $0.6586^{\circ} \mathrm{S}, 76.4521^{\circ} \mathrm{W}, 220-250 \mathrm{~m}, 30$ September 1996 (TL Erwin, et al.)(NMNH: ADP137337, male paratype), Erwin Piraña Plot: transect 5, station 10, $0.6566^{\circ} \mathrm{S}$, $76.4490^{\circ} \mathrm{W}, 220-250 \mathrm{~m}, 2$ October 1996 (TL Erwin, et al.)(NMNH: ADP137750, female paratype), Erwin Piraña Plot: transect 5, station 8, $0.6566^{\circ} \mathrm{S}, 76.4490^{\circ} \mathrm{W}, 220$ 250m, 6 October 1996 (TL Erwin, et al.)(NMNH: ADP135857, female paratype); Yasuni National Park (edge), $95.43 \mathrm{~km} \mathrm{E} \mathrm{(heading} \mathrm{101.46})$ Coca, Tiputini Biodiversity Station, Erwin Harpia Plot: transect 1, station 6, $0.6342^{\circ} \mathrm{S}, 76.1443^{\circ} \mathrm{W}, 218 \mathrm{~m}$, 23 October 1998 (TL Erwin, et al.)(NMNH: ADP135902, ADP135874, female paratypes), Erwin Harpia Plot: transect 3, station 9, $0.63327^{\circ} \mathrm{S}, 76.1443^{\circ} \mathrm{W}, 207 \mathrm{~m}$, 24 October 1998 (TL Erwin, et al.)(NMNH: ADP135843, female paratype), Erwin Harpia Plot: transect 6, station 9, 0.6295' S, 76.1443ํ, 199m, 26 October 1998 (TL Erwin, et al.)(NMNH: ADP135850, male paratype), Erwin Harpia Plot: transect 9, station 8, $0.6269^{\circ} \mathrm{S}, 76.1443^{\circ} \mathrm{W}, 203 \mathrm{~m}, 5$ February 1999 (TL Erwin, et al.) (NMNH: ADP135844, male paratype, ADP135853, ADP135898, female paratypes), Erwin Harpia Plot: transect 6, station 7, 0.6295 S, 76.1443W, 199m, 7 February 1999 (TL Erwin, et al.)(NMNH: ADP135849, male paratype), Erwin Harpia Plot: transect 4 , station $4,0.6316^{\circ} \mathrm{S}, 76.1443^{\circ} \mathrm{W}, 208 \mathrm{~m}, 8$ February 1999 (TL Erwin, et 
al.)(NMNH: ADP135851, ADP135845, male paratypes), Erwin Harpia Plot: transect 6, station 9, $0.6295^{\circ} \mathrm{S}, 76.1443^{\circ} \mathrm{W}, 199 \mathrm{~m}, 25$ October 1999 (TL Erwin, et al.) (NMNH: ADP135916, female paratype). Perú, Loreto, Pacaya-Samiria National Reserve, $1 \mathrm{~km}$ E Hamburgo, Boca del Ingles Camp, 0,5226 $\mathrm{S}, 75.1192^{\circ} \mathrm{W}, 125 \mathrm{~m}, 10$ May 1990 (TL Erwin, et al.)(NMNH: ADP005785, female paratype); Río Samiria (South Branch), Camp Terry, 5.6951으, 75.2243W, 129m, 12 May 1990 (TL Erwin, et al.)(NMNH: ADP005786, female paratype); MADre De Dios, Manu Reserved Zone, Río Manu, BIOLAT Biological Station, Pakitza, $11.9446^{\circ} \mathrm{S}, 71.2831^{\circ} \mathrm{W}, 356 \mathrm{~m}$, 7 September 1988 (TL Erwin, et al.)(NMNH: ADP005778, ADP005779, male paratypes), 9 September 1988 (TL Erwin, et al.)(NMNH: ADP005763, female paratype), 12 September 1988 (TL Erwin, et al.)(NMNH: ADP005776, male paratype), 15 February 1990 (TL Erwin, GP Servat)(NMNH: ADP005777, male paratype), 21 September 1991 (TL Erwin, et al.)(NMNH: ADP005764, male paratype), 16 October 1991 (TL Erwin, MG Pogue)(NMNH: ADP007444, male paratype), Reserva Nacional Tambopata, $30 \mathrm{~km}$ (air) SW Puerto Maldonado, Explorer's Inn, $12.8364^{\circ} \mathrm{S}$, 69.2936 W, 209m, 14 September 1984 (TL Erwin, et al.)(NMNH: ADP005781, female paratype), 25 October 1982 (TL Erwin, et al.)(NMNH: ADP005798, female paratype), 5-6 November 1982 (R Wilkerson)(NMNH: ADP067693, male paratype), 30 April 1984 (TL Erwin, et al.)(NMNH: ADP005784, male paratype), 8 September 1984 (TL Erwin, et al.)(NMNH: ADP005783, female paratype), 14 September 1984 (TL Erwin, et al.)(NMNH: ADP005782, male paratype).

Geographic distribution (Fig. 11). This species is currently known from the type locality and other localities in Ecuador and various localities in Colombia and Perú.

Notes. The holotype will be deposited in Escuela Politécnica Nacional, Quito, Ecuador and is currently held in trust until the completion of studies at NMNH.

\section{Hyboptera viridivittis Chaudoir, 1872}

Green-lined humps-backed beetle

Figs 4B, 5E, 11

Hyboptera viridivittis Chaudoir, 1872: 164.

Lectotype. (Here designated) Sex unknown. Brazil, Rio de Janeiro, Cantagallo (RF Sahlberg)(MNHP).

Derivation of specific epithet. The specific epithet, viridivittis, is a feminine Latin adjective referring to the green line pattern of the pronotum.

Proposed English vernacular name. Green-lined humps-backed beetle.

Diagnosis. With the attributes of the genus and angulicollis species group as described above and adults with patches of bright metallic green para-medially on pronotum, elytra dark matte black, some individuals with hint of metallic green near basal margin. Venter with gular region, prosternal region, meso- and metathroax, and margins of abdominal sterna infuscated, otherwise rufous. 
Description. (Figs 4B, 5E). Size: See Appendix 1. Length (SBL) medium for genus, $\mathrm{ABL}=4.65-5.27 \mathrm{~mm}, \mathrm{SBL}=3.76-4.17 \mathrm{~mm}, \mathrm{TW}=2.18-2.59 \mathrm{~mm}$.

Color: See diagnosis, above.

Luster: Matte, pronotum and elytra with metallic highlights.

Microsculpture: Mostly isodiametric or stretched, shallowly impressed, cells more stretched around elytral tubercles.

Head: Rugae moderately coarse, mostly transverse or angulate. Eye large, sub-hemispheric, evenly rounded anteriorly, subtly more prolonged posteriorly. Antenna short, barely reaching humerus. Labrum rectangulate, shallowly bilobed, anterior margin slightly emarginate. Neck finely transversely rugose.

Prothorax: Pronotum moderately broad, disc centrally depressed along midline with dense transverse rugae. Lateral margins broadly explanate and obtusely angulate medially then moderately arcuate to obtuse hind angle, base medially produced and rounded posteriorly.

Pterothorax: Normal for Agrina, fully winged. Elytron interval 3 with 8 and interval 5 with 6 unisetiferous tubercles, other intervals moderately convex, side margin narrowly explanate laterally, slightly more so medially. Elytron moderately broad and short, slightly narrower than the pronotum at the broadest part, apex truncate, slightly rounded with distal corner broadly and obtusely rounded, disc not significantly convex, basal third slightly depressed. All interneurs well-impressed.

Legs: Femur dorso-ventrally moderately depressed, tibia coequal in length, more depressed; tarsus less than half the length of the tibia, fourth tarsomere markedly bilobed and with tarsal pad of setae.

Abdomen: Sparsely setiferous; normal ambulatory setae on sterna 3-5; female with two pairs of ambulatory setae on sternum 6 , medial pair of setae less than the length of lateral pair; males with only the lateral pair of longer setae.

Male genitalia: Phallus (Fig. 5E) with ostium of 1/5 its length, catopic, apex moderately long, narrowly rounded, broad in dorsal aspect; endophallus with flagellum (mid-part obvious in illustration), flagellum not barbed. Parameres asymmetric, right very small, left larger.

Female genitalia: Not investigated, likely similar to that of $H$. lucida (Fig. 8).

Dispersal potential. These beetles are macropterous and probably capable of flight. They are moderately swift and agile runners.

Way of life. Adults are found in April-June, the early dry season, in lowlands (75846 m.a.s.l.) in the Mata Atlântica.

Other specimens examined. Brazil, Rio de JANeIro, Rio de Janeiro, $22.9522^{\circ} \mathrm{S}$, $43.2109^{\circ} \mathrm{W}, 459 \mathrm{~m}, 1883$ (P Germain)(NMNH: ADP136142, female), SANTA Catarina, Nova Teutonia, 27.1833 $\mathrm{S}, 52.3833^{\circ} \mathrm{W}, 300-500 \mathrm{~m}$, April 1977 (F Plaumann)(CAS: ADP007617, male), May 1977 (F Plaumann)(CAS: ADP007597, ADP007601, males, ADP007599, ADP007618, females), June 1977 (F Plaumann) (CAS: ADP007600, ADP007616, females).

Geographic distribution (Fig. 11). This species is currently known from the states of Minas Gerais, Rio de Janeiro, Santa Catarina, and São Paulo in the Mata Atlântica of Brazil. 
Notes. Reichardt $(1971,1973)$ reported the following additional specimens that we did not see: Brazil - Minas Gerais: no locality (2 exs. MNHP). Rio de Janeiro: Nova Friburgo (l ex. BMNH). Guanabara, (5 exs. BMNH, MNHP, MZSP). Sáo Paulo: Barueri (l ex. MZSP). Santa Catarina: Nova Teutonia (1 ex. BMNH), Corupá (1 ex. MCZ). Additionally, Chaudoir (1872) mentioned he had two specimens; therefore, a lectotype needs to be designated. Reichardt (1973) mistakenly writes he saw the "holotype." We have chosen the first of Chaudoir's specimens as the Lectotype (see above).

\section{tuberculata species group}

(recognized by Reichardt 1973)

The most distinctive attribute of species in this group is that the pronotum has discal rugae etched at an angle aimed medio-posteriorly, or somewhat chaotically seemingly without order. Adults of all species have no metallic coloration (except $H$. apollonia with subtle traces only) on the dorsal surface and the general adult size is medium to large for the genus. Male phallus apex short, broadly or narrowly blunt.

\section{Hyboptera apollonia Erwin, 2004}

Apollonia's humps-backed beetle

Figs 6A, 10A, 11

Hyboptera apollonia Erwin, 2004: 33.

Holotype. Male. Panamá, Colón, 30 km NE Colón, Porto Bello, $9.555^{\circ} \mathrm{N}, 79.653^{\circ} \mathrm{W}$, 9m, 23 February 1911)(EA Schwarz)(NMNH: ADP007943).

Derivation of specific epithet. The specific epithet, apollonia, is an eponym based on the first name of Michael Corleone's beautiful young Italian wife in the movie The Godfather whose death in a car explosion perpetrated by Mafia competition signifies the useless instantaneous death of so many species when humans put fire to the tropical rain forest in time of drought.

Proposed English vernacular name. Apollonia's humps-backed beetle.

Diagnosis. With the attributes of the genus and angulicollis species group as described above and with only dark non-metallic markings on the pronotal disc; pronotum with discal rugae etched horizontally and linear. Elytra black with paler margin not reaching suture. Mouthparts, appendages, margin of prothorax, venter of head and prothorax, abdominal segments II-V testaceous; meso- and metathorax, and abdominal segment VI infuscated.

Description. (Figs 6A, 10A). Size: See Appendix 1. Length (SBL) medium for genus, $\mathrm{ABL}=4.19-5.14 \mathrm{~mm}, \mathrm{SBL}=3.53-4.26 \mathrm{~mm}, \mathrm{TW}=2.09-2.57 \mathrm{~mm}$. 


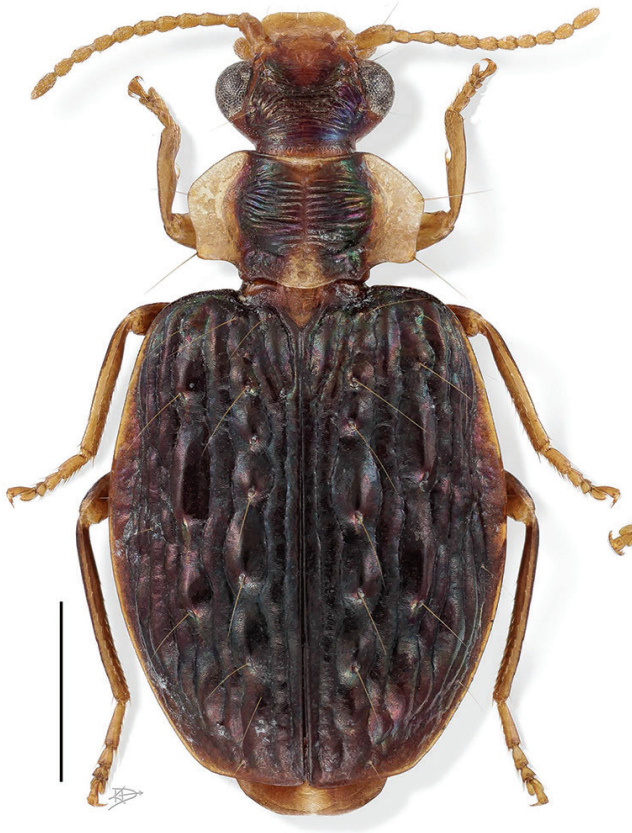

A

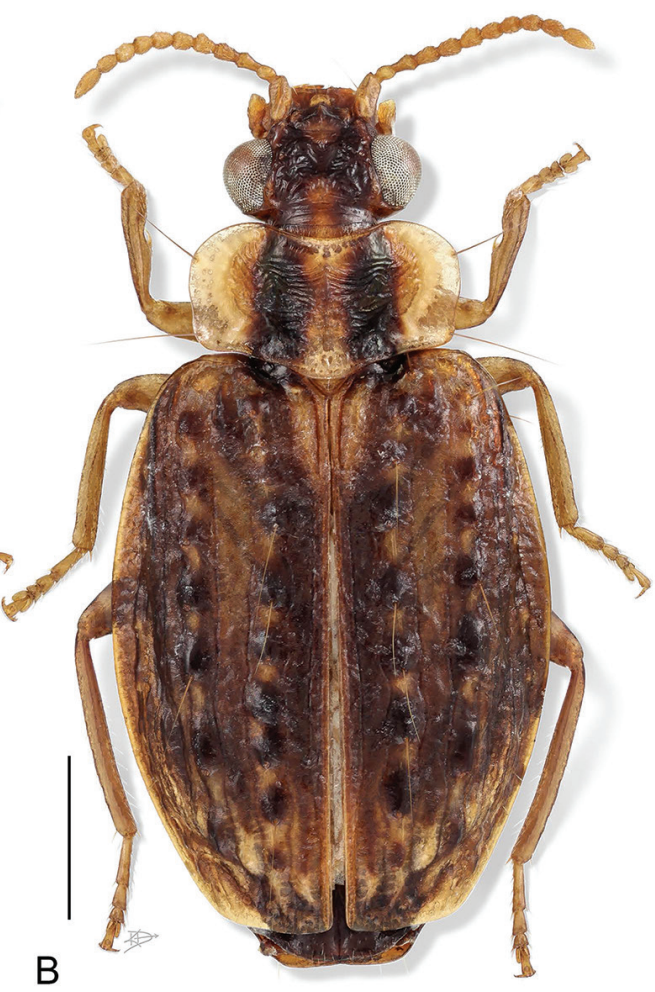

Figure 6. Digital Photo-illustration. Habitus, dorsal aspect. A Hyboptera apollonia Erwin, male, ADP007944 B Hyboptera auxilidora Erwin, male, ADP007622.

Color: See diagnosis, above.

Luster: Shiny, no metallic highlights, subtle iridescent around tubercles.

Microsculpture: Mostly isodiametric or slightly stretched, well-impressed, cells somewhat stretched around elytral tubercles.

Head: Rugae moderately coarse, mostly not arranged. Eye very large, sub-hemispheric, evenly rounded anteriorly, subtly more prolonged posteriorly. Antenna short, barely reaching humerus. Labrum rectangulate, shallowly bilobed, anterior margin slightly emarginate. Neck finely transversely rugose.

Prothorax: Pronotum markedly broad, disc centrally slightly depressed with dense transverse rugae. Lateral margins very broadly explanate and obtusely rounded medially then nearly straight to obtuse hind angle, base medially produced and rounded posteriorly.

Pterothorax: Normal for Agrina, fully winged. Elytron intervals 3 with 7, and interval 5 with 6 (5) discal unisetiferous tubercles, other intervals moderately convex, side margin moderately explanate laterally only at middle third. Elytron broad and moderately short, moderately narrower than the pronotum at the broadest part, apex truncate, slightly rounded with distal corner broadly and obtusely rounded, disc not significantly convex, basal third slightly depressed. All interneurs well-impressed. 
Legs: Femur dorso-ventrally moderately depressed, tibia coequal in length, more depressed; tarsus less than half the length of the tibia, fourth tarsomere markedly bilobed and with tarsal pad of setae.

Abdomen: Sparsely setiferous; normal ambulatory setae on sterna 3-5; female with two pairs of ambulatory setae on sternum 6 , medial pair of setae less than the length of lateral pair; males with only the lateral pair of longer setae on sternum 6 .

Male genitalia: Phallus (Fig. 10A) with ostium of $1 / 6$ its length, catopic, apex very short, moderately rounded; endophallus with flagellum (base obvious in illustration), flagellum not barbed. Parameres asymmetric, right very small, left larger.

Female genitalia: Not investigated, likely similar to that of H. lucida (Fig. 8).

Dispersal potential. These beetles are macropterous and capable of flight. They are moderately swift and agile runners. Modern collecting methods have acquired specimens, including insecticidal foggings and malaise traps.

Way of life. An adult of this species was fogged from a tree in the genus Guarea at La Selva and another caught in a Malaise trap as part of the ALAS Project. F. Nevermann collected a specimen on a rotten log. The known elevational range of this species is between 9 and 815 m.a.s.l. Adults have been obtained in January-April, and September-October; hence they are active in both the dry and rainy seasons in the lowlands and lower middle altitudes, both on the east and west sides of the Cordillera Central.

Other specimens examined. Costa Rica, Heredia, $11 \mathrm{~km}$ SE La Virgin, $10.4313^{\circ} \mathrm{N}, 84.0056^{\circ} \mathrm{W}, 450-650 \mathrm{~m}, 11$ May 2003 (Proy. ALAS)(INBIO: ADP135881, female), 250-350m, 22 February 2004 (Proy. ALAS)(INBIO: ADP140501, female), $3 \mathrm{~km} \mathrm{~S} \mathrm{Pto.} \mathrm{Viejo,} \mathrm{Estación} \mathrm{Biológica} \mathrm{La} \mathrm{Selva,} 10.4313^{\circ} \mathrm{N}, 84.0056^{\circ} \mathrm{W}, 150 \mathrm{~m}$, 18 May 1993, (Proy. ALAS)(HESP: ADP102312, female), 13 January 1996 (Proy. ALAS)(INBIO-OET: ADP135879, female); Limón, Rio Reventazon, Ebene, Hamburg Farm, $10.4149^{\circ} \mathrm{N}, 83.7506^{\circ} \mathrm{W}, 50 \mathrm{~m}, 4$ October 1928 (F Nevermann)(NMNH: ADP007942, female), 20 April 1932 (F Nevermann)(NMNH: ADP007945, female), P.N. Tortuguero, Tortuguero, Estación Cuatro Esquinas, $10.5338^{\circ} \mathrm{N}, 83.5071^{\circ} \mathrm{W}$, sea level, June 1991 (J Solano)(INBIO, 007578, male); Puntarenas, Peninsula de Osa, Rancho Quemado, 8.6790 W, 83.5667 W, 200m, September 1991 (F Quesada)(INBIO: ADP100266, male). Panamá, Colón, $30 \mathrm{~km} \mathrm{NE} \mathrm{Colón,} \mathrm{Porto} \mathrm{Bello,} 9.555^{\circ} \mathrm{N}$, 79.653W, 9m, 2-11 March 1911 (EA Schwarz)(NMNH: ADP007944, male), Gamboa, Pipeline Road, 9.124 N, 79.749 $\mathrm{W}, 37 \mathrm{~m}, 17-22$ June 1993 (SW Lingafelter) (SEMC: ADP007577, female); Panamá, Cerro Jefe, $9.2373^{\circ} \mathrm{N}, 79.3549^{\circ} \mathrm{W}, 815 \mathrm{~m}, 20$ May 1972 (RT Allen)(NMNH: ADP011167, female).

Geographic distribution (Fig. 11). This species is currently known from the type locality in Panamá and throughout southern Central America north to Costa Rica.

Notes. Adults of this species are unusual in that they subtly bear two attributes similar to species in the angulicollis species-group: faint traces of metallic green and a short narrow nubnen-like apex of the phallus, rather than short and broadly blunt. Adults of the angulicollis species-group are extensively green and the phallus apex, while narrow is elongate. 


\section{Hyboptera auxilidora Erwin, 2004}

Maria's humps-backed beetle

Figs 6B, 11

Hyboptera auxilidora Erwin, 2004: 35.

Holotype. Male. USA. Texas, Hidalgo County, nr. Mission, Bentson Rio Grande State Park, $26.176^{\circ} \mathrm{N}, 98.385^{\circ} \mathrm{W}, 38 \mathrm{~m}, 18$ July 1981 (WE Steiner)(NMNH: ADP007623).

Derivation of specific epithet. The specific epithet, auxiliadora, is an eponym based on the middle name of María Auxiliadora Sanchez, who for many years was responsible for the welfare of participating visiting taxonomists at INBio and its facilities and who made life easy therein while we undertook our studies of the rich Costa Rican fauna and flora.

Proposed English vernacular name. Maria’s humps-backed beetle.

Diagnosis. With the attributes of the genus and tuberculata species group as described above and adults with only dark non-metallic markings on the pronotal disc, venter substantially infuscated, pronotum with discal rugae etched at an angle aimed medio-posteriorly, or somewhat chaotically; elytron with sutural margin at apical sixth pale, not black, if brownish not contrasting with background color, and elytra just posterior to scutellum with a V-shaped pale area encompassing the sutures and first intervals. Elytron broad with markedly arcuate lateral margin.

Description. (Fig. 6B). Size: See Appendix 1. Length (SBL) long for genus, ABL $=5.06-5.93 \mathrm{~mm}, \mathrm{SBL}=4.39-4.98 \mathrm{~mm}, \mathrm{TW}=2.42-3.28 \mathrm{~mm}$.

Color: See diagnosis, above.

Luster: Shiny, not metallic.

Microsculpture: Mostly isodiametric or stretched, shallowly impressed, cells somewhat stretched around elytral tubercles.

Head: Rugae moderately coarse, mostly chaotic. Eye markedly large, sub-hemispheric, evenly rounded anteriorly, subtly more prolonged posteriorly. Antenna short, barely reaching humerus. Labrum rectangulate, shallowly bilobed, anterior margin slightly emarginate. Neck transversely rugose.

Prothorax: Pronotum markedly broad, disc centrally depressed along midline with dense arcuate rugae directed anteriorly in apical half, posteriorly in posterior half of disc. Lateral margins broadly explanate and subtly produced at lateral seta, but not acute medially then straight to obtuse hind angle, base medially slightly produced and rounded posteriorly.

Pterothorax: Normal for Agrina, fully winged. Elytron interval 3 with 10 discal unisetiferous tubercles and interval 5 with 9(8) discal unisetiferous tubercles, interval 3 with one such tubercle near apex, other intervals moderately convex, side margin broadly explanate laterally at middle third. Elytron moderately broad and short, moderately narrower than the pronotum at the broadest part, apex truncate, slightly rounded with distal corner broadly and obtusely rounded, disc not significantly convex, basal third slightly depressed. All interneurs well-impressed. 
Legs: Femur dorso-ventrally moderately depressed, tibia coequal in length, more depressed; tarsus less than half the length of the tibia, fourth tarsomere markedly bilobed and with tarsal pad of setae.

Abdomen: Sparsely setiferous; normal ambulatory setae on sterna 3-5; female with two pairs of ambulatory setae on sternum 6 , medial pair of setae less than the length of lateral pair; males with only the lateral pair of longer setae.

Male genitalia: Phallus (see fig. 20 in Erwin, 2004) with ostium 1/4 length, catopic and apex short and evenly rounded, endophallus with flagellum, flagellum not barbed. Parameres asymmetric, right very small, left very large.

Female genitalia: Not investigated, likely similar to that of $H$. lucida (Fig. 8).

Dispersal potential. These beetles are macropterous and capable in flight. They are moderately swift and agile runners. Specimes have been collected from light traps (white and UV light) and Malaise traps.

Way of life. Adults are found in July in lowlands (10-240 m.a.s.l.) in terra firme forests. The holotype was collected by W. Steiner from under bark of the tree Celtis levigata Willd. Vogt collected another individual from under the web tent of a psocid colony (Psocoptera). Adults have been obtained in March, April, May, July, August, and September; hence they are active in both the dry and rainy seasons in the lowlands on both sides of the Cordillera Central.

Other specimens examined. Costa Rica, Guanacaste, P.N. Guanacaste, 30 km N Liberia, Finca Jenny, 10.8655N, 85.5735 W, 240m, 14-21 August 1993 (E Araya)(INBIO: ADP007640, female); Limón, Rio Reventazon, Ebene, Hamburg Farm, $10.4149^{\circ} \mathrm{N}, 83.7506^{\circ} \mathrm{W}, 50 \mathrm{~m}, 6$ November 1925 (F Nevermann) (NMNH: ADP007947, female); Puntarenas, P.N. Carara, Estación Quebrada Bonita, $9.7737^{\circ} \mathrm{N}, 84.6122^{\circ} \mathrm{W}, 100 \mathrm{~m}, 17$ March - 30 April ---- (P Campos)(INBIO: ADP007639, male). Honduras, Atlantida, Tela, $15.719^{\circ} \mathrm{N}, 87.458^{\circ} \mathrm{W}$, 85m, 15 May 1995 (R Cave)(NMNH: ADP135859, female). México, VeracRUZ, $3.58 \mathrm{~km}$ NE Catemaco, Lake Catemaco, $18.444 \mathrm{~N}, 95.078 \mathrm{~W}, 355 \mathrm{~m}, 23$ August 1967 (GE Ball, TL Erwin, et al.)(UASM: ADP007621, female). Panamá, Canal Zone, Barro Colorado Nature Monument, Barro Colorado Island, Barro Colorado Research Station, Mess Hall, 9.1652 N, 79.8368 W, 70m, 19 February 1975 (TL Erwin, JL Lawrence)(NMNH: ADP027570, male); Cocle, El Valle, $8.603^{\circ} \mathrm{N}, 80.152^{\circ} \mathrm{W}, 829 \mathrm{~m}, 26$ May 1983 (WE Steiner)(NMNH: ADP135861, ADP135863, males, ADP135865, ADP005801, females). USA, TExAs, southeast Hidalgo County, 1 December 1946 (GB Vogt)(NMNH: ADP007638, female), Bentson Rio Grande State Park, Mission, $26.176^{\circ} \mathrm{N}, 98.385^{\circ} \mathrm{W}, 38 \mathrm{~m}, 16$ July 1975 (CA Triplehorn)(OSU: ADP007622, female), Sabal Palm Grove Sanctuary, nr. Southmost, $25.8419^{\circ} \mathrm{N}, 97.4247^{\circ} \mathrm{W}, 8 \mathrm{~m}, 1$ May 1979 (R.Turnbow)(UASM: ADP007620, male).

Geographic distribution (Fig. 11). This species is currently known only from the type locality and nearby areas in Texas, and from Panamá, and in between those extremes only from México, Honduras, and Costa Rica. It likely is to be found in other Central American countries with further sampling. 


\section{Hyboptera dilutior Oberthür, 1884}

Oberthür's humps-backed beetle

Figs 7A, 10B, 11

Hyboptera dilutior Oberthür, 1884: 52.

Holotype. Sex unknown. Brazil. Amazonas: Tefé (MNHP).

Derivation of specific epithet. The specific epithet, dilutior, is an adjective referring to the "washed out" appearance of the color of adults of this species in comparison with those of other species.

Proposed English vernacular name. Oberthür's humps-backed beetle.

Diagnosis. With the attributes of the genus and tuberculata species group as described above and adults with only dark non-metallic markings on the head and pronotal disc, pronotum with discal rugae in basal half etched at an angle aimed medioposteriorly, or somewhat chaotically. Elytra mostly testaceous with darkly marked tubercles; small medio-apical tubercles also infuscated; with sutural margin at apical sixth infuscated markedly contrasting with testaceous background color. Venter of head and thorax substantially infuscated; abdominal sterna mostly pale with subtle infuscation.

Description. (Figs 7A, 10B). Size: See Appendix 1. Length (SBL) long for genus, $\mathrm{ABL}=3.92-5.98 \mathrm{~mm}, \mathrm{SBL}=3.55-4.67 \mathrm{~mm}, \mathrm{TW}=1.95-3.21 \mathrm{~mm}$.

Color: See diagnosis, above.

Luster: Without any metallic highlights, shiny.

Microsculpture: Mostly isodiametric and stretched, shallowly impressed, cells somewhat more stretched around elytral tubercles.

Head: Rugae moderately coarse, mostly without patterned arrangement. Eye moderately large, hemispheric, evenly rounded. Antenna short, barely reaching humerus. Labrum rectangulate, shallowly bilobed, anterior margin slightly emarginate. Neck finely transversely rugose.

Prothorax: Pronotum markedly broad, disc centrally markedly depressed with coarse angulate rugae. Lateral margins broadly explanate and evenly rounded to obtuse hind angle, base medially produced and rounded posteriorly.

Pterothorax: Normal for Agrina, fully winged. Elytron intervals 3 and 5 each with 7 promient discal unisetiferous tubercles, other intervals moderately convex, side margin broadly explanate laterally only at middle third. Elytron broad and short, much narrower in width to that of the pronotum at the broadest part, apex truncate, slightly rounded with distal corner broadly and obtusely rounded, disc not significantly convex, basal third slightly depressed. All interneurs well-impressed.

Legs: Femur dorso-ventrally moderately depressed, tibia coequal in length, more depressed; tarsus less than half the length of the tibia, fourth tarsomere markedly bilobed and with tarsal pad of setae.

Abdomen: Sparsely setiferous; normal ambulatory setae on sterna 3-5; female with two pairs of ambulatory setae on sternum 6 , medial pair of setae less than the length of lateral pair; males with only the lateral pair of longer setae. 


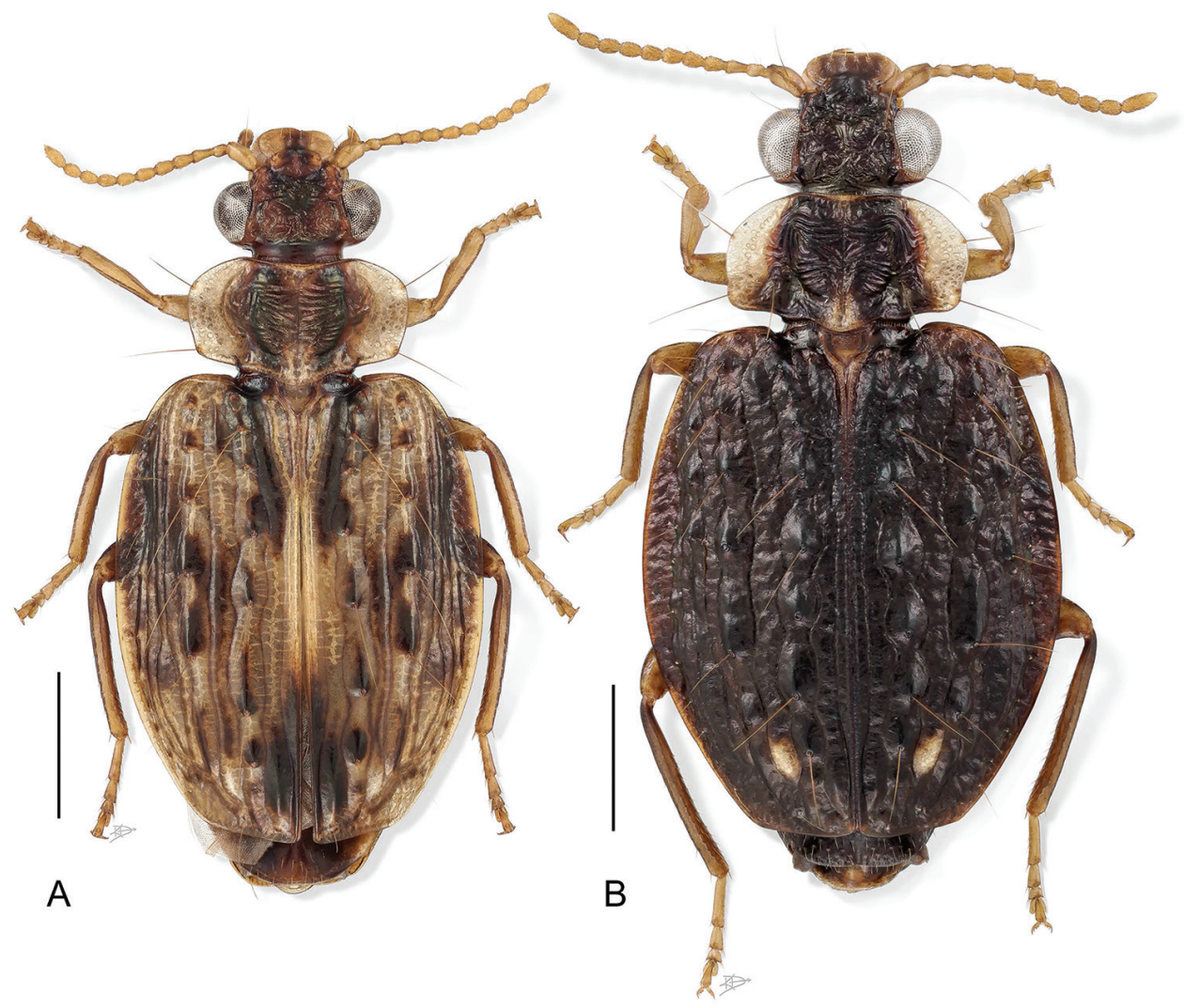

Figure 7. Digital Photo-illustration. Habitus, dorsal aspect. A Hyboptera dilutior Oberthür, male, ADP094021 B Hyboptera lucida Henry \& Erwin, sp. n., female, ADP135783.

Male genitalia: Phallus (Fig. 10B) with ostium of $1 / 6$ its length, catopic, apex very short, narrowly pointed, broadly rounded in dorsal aspect; endophallus with flagellum (obvious in illustration), flagellum not barbed. Parameres asymmetric, right very small, left larger.

Female genitalia: Not investigated, likely similar to that of $H$. lucida (Fig. 8).

Dispersal potential. These beetles are macropterous and capable of flight. They are moderately swift and agile runners. Adults of this species are attracted to C.D.C. light traps and captured in SLAM Malaise traps.

Way of life. Adults are common in the lowlands (7 to $356 \mathrm{~m}$ a.s.l.) and appear to be generalists in a variety of rainforest biotopes including terra firme and várzea. In these forests, they are commonly found in big trees with vines and epiphytes, in suspended dry leaves, in dry Sheelea sp. and Astrocaryum chambira Burret palm frond skirts. Individuals can be found from January to December, in both the rainy and dry seasons. Member of this species have been recorded from the canopy of the following tree species using insecticidal fogging techniques: Chrysophyllum argenteum cf.; Sterculia colombiana cf.; Parkia multijuga cf.; Naucleopsis herrerensis cf.; Matisia malacoca- 
lyx cf.; Pseudolmedia laevigata; Cecropia herthae; Pentagonia spathicalyx cf.; Eschweilera coriacea cf.; Batocarpus orinocensis cf.; Zanthoxylum riedelianum ssp. kellermanii cf.; Cecropia ficifolia; Inga capitata; Leonia glycicarpa; Coussapoa herthae; Pourouma mollis ssp. triloba cf.; Pausandra trianae; Coussapoa orthoneura cf.; Alchornea triplinervia cf.; Protium sagotianum cf.; Guatteria sp.; Meiocarpus, long petiole; Oenocarpus bataua; Neea dive-tuberculate; Semaphyllanthe megistocaula cf.; Lauraceae redvein; Siparuna decipiens; Trichilia solitudinis.

Other specimens examined. BraziL, Amazonas, Parana do Xiboreninho, $3.2482^{\circ} \mathrm{S}, 59.9791^{\circ} \mathrm{W}, 7 \mathrm{~m}, 7$ August 1979 (TL Erwin, J Adis, et al.)(NMNH:

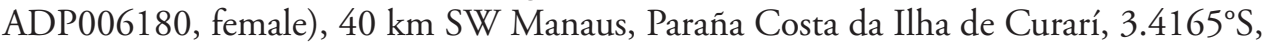
$60.2508^{\circ} \mathrm{W}, 17 \mathrm{~m}, 3$ August 1979 (TL Erwin, J Adis)(NMNH: ADP005799, male), north of Manaus, on Amazonas 010 at $\mathrm{Km} 26$, Reserva Ducke, $2.918^{\circ} \mathrm{S}, 59.971^{\circ} \mathrm{W}$, 70m, 2 May 1978 (J Arias)(NMNH: ADP135826, female), 11 May 1978 (J Arias) (NMNH: ADP135786, male), 1 August 1978 (J Arias)(NMNH: ADP135804, female), 6 September 1978 (J Arias)(NMNH: ADP135792, male), 13 September 1978 (J Arias)(NMNH: ADP135790, ADP135818, females), 20 September 1978 (J Arias) (NMNH: ADP135778, male); PARÁ, Belém, $1.555^{\circ} \mathrm{S}, 48.429^{\circ} \mathrm{W}, 25 \mathrm{~m}, 25$ January 1969 (L O’Brien, CW O’Brien)(NMNH: ADP006178, female), Parque Estadual do Utinga, Belém, $1.406^{\circ} \mathrm{S}, 48.399^{\circ} \mathrm{W}, 21 \mathrm{~m}, 20$ January 1977 (N Guimaraes)(NMNH: ADP006179, female); Rondonia, $7 \mathrm{~km}$ E Costa Marques, $12.435^{\circ} \mathrm{S}, 64.292^{\circ} \mathrm{W}$, 153m, 11-23 April 1987 (T Klein)(NMNH: ADP135883, female). Ecuador, Orellana, Reserva Ethnica Huaorani, $39 \mathrm{~km}$ S, Pompeya, Estación Científica Yasuní Onkone Gare Camp, Erwin Piraña Plot: transect 4, station 7, $0.6570^{\circ} \mathrm{S}, 76.4498^{\circ} \mathrm{W}$, 220-250m, 16 January 1994 (TL Erwin, et al.)(NMNH: ADP137700, female), Erwin Piraña Plot: transect 5, station 7, $0.6566^{\circ} \mathrm{S}, 76.4490^{\circ} \mathrm{W}, 220-250 \mathrm{~m}, 26$ January 1994 (TL Erwin, et al.)(NMNH: ADP135940, male), Erwin Piraña Plot: transect 10, station $10,0.6540^{\circ} \mathrm{S}, 76.4453^{\circ} \mathrm{W}, 220-250 \mathrm{~m}, 8$ February 1995 (TL Erwin, et al.) (NMNH: ADP006175, male), Erwin Piraña Plot: transect 8, station 5, 0.6551 ${ }^{\circ} \mathrm{S}$, 76.4403ํW, 220-250m, 30 June 1995 (TL Erwin, et al.)(NMNH: ADP137756, male), Erwin Piraña Plot: transect 1, station 4, 0.6586 ${ }^{\circ}$, $76.4521^{\circ} \mathrm{W}, 220-250 \mathrm{~m}, 4$ February 1996 (TL Erwin, et al.)(NMNH: ADP137692, female), Erwin Piraña Plot: transect 5, station $7,0.6566^{\circ} \mathrm{S}, 76.4490^{\circ} \mathrm{W}, 220-250 \mathrm{~m}, 9$ February 1996 (TL Erwin, et al.)(NMNH: ADP135974, male), Erwin Piraña Plot: transect 2, station 7, $0.6581^{\circ} \mathrm{S}$, 76.4513º W, 220-250m, 30 September 1996 (TL Erwin, et al.)(NMNH: ADP137698, male), Erwin Pirańa Plot: transect 6, station 2, $0.6561^{\circ} \mathrm{S}, 76.4483^{\circ} \mathrm{W}, 220-250 \mathrm{~m}, 2$ October 1996 (TL Erwin, et al.)(NMNH: ADP135926, female), Erwin Piraña Plot: transect 8 , station $5,0.6551^{\circ} \mathrm{S}, 76.4403^{\circ} \mathrm{W}, 220-250 \mathrm{~m}, 3$ October 1996 (TL Erwin, et al.)(NMNH: ADP135930, male), Erwin Piraña Plot: transect 9, station 8, $0.6545^{\circ} \mathrm{S}$, 76.4460 ${ }^{\circ} \mathrm{W}, 220-250 \mathrm{~m}, 4$ October 1996 (TL Erwin, et al.)(NMNH: ADP137387, ADP137690, males), Erwin Piraña Plot: transect 3, station $7,0.6575^{\circ} \mathrm{S}, 76.4505^{\circ} \mathrm{W}$, 220-250m, 21 January 2006 (TL Erwin, et al.)(NMNH: ADP133846, male), Yasuni National Park (edge), $95.43 \mathrm{~km}$ E (heading $101.46^{\circ}$ ) Coca, Tiputini Biodiversity Station, Erwin Harpia Plot: transect 10, station 2, $0.6262^{\circ} \mathrm{S}, 76.1443^{\circ} \mathrm{W}, 214 \mathrm{~m}, 5$ Febru- 
ary 1999 (TL Erwin, et al.)(NMNH: ADP135832, female), Orellana, Yasuni National Park (edge), $95.43 \mathrm{~km}$ E (heading $101.46^{\circ}$ ) Coca, Tiputini Biodiversity Station, Erwin Harpia Plot: transect 3, station 9, $0.6332^{\circ} \mathrm{S}, 76.1443^{\circ} \mathrm{W}, 207 \mathrm{~m}, 30$ June 1998 (TL Erwin, et al.)(NMNH: ADP135782, female), Erwin Harpia Plot: transect 10, station 8, $0.6262^{\circ} \mathrm{S}, 76.1443^{\circ} \mathrm{W}, 214 \mathrm{~m}, 21$ October 1998 (TL Erwin, et al.)(NMNH: ADP135836, male), Erwin Harpia Plot: transect 10, station 3, $0.6262^{\circ} \mathrm{S}, 76.1443^{\circ} \mathrm{W}$, 214m, 5 February 1999 (TL Erwin, et al.)(NMNH: ADP135784, female), Erwin Harpia Plot: transect 6, station 5, $0.6295^{\circ} \mathrm{S}, 76.1443^{\circ} \mathrm{W}, 199 \mathrm{~m}, 7$ February 1999 (TL Erwin, et al.)(NMNH: ADP135840, male, ADP135806, female), Erwin Harpia Plot: transect 5, station $6,0.6332^{\circ} \mathrm{S}, 76.1443^{\circ} \mathrm{W}, 207 \mathrm{~m}, 8$ February 1999 (TL Erwin, et al.) (NMNH: ADP135794, female), Erwin Harpia Plot: transect 10, station 4, $0.6262^{\circ} \mathrm{S}$, $76.1443^{\circ} \mathrm{W}, 214 \mathrm{~m}, 28$ September 2000 (TL Erwin, et al.)(NMNH: ADP139140, male), Erwin Harpia Plot: transect 6, station $1,0.6304^{\circ} \mathrm{S}, 76.1443^{\circ} \mathrm{W}, 199 \mathrm{~m}, 1$ October 2000 (TL Erwin, et al.)(NMNH: ADP139128, ADP139134, ADP139150, males), Erwin Harpia Plot: transect 1, station 3, $0.6342^{\circ}$ S, $76.1443^{\circ} \mathrm{W}, 214 \mathrm{~m}, 2$ October 2000 (TL Erwin, et al.)(NMNH: ADP139124, male), Erwin Harpia Plot: transect 10, station 5, $0.6262^{\circ} \mathrm{S}, 76.1443^{\circ} \mathrm{W}, 214 \mathrm{~m}, 17$ February 2001 (TL Erwin, et al.)(NMNH: ADP139112, male), Erwin Harpia Plot: transect 3, station $1,0.6332^{\circ} \mathrm{S}, 76.1443^{\circ} \mathrm{W}$, 207m, 24 July 2001 (TL Erwin, et al.)(NMNH: ADP139527, female); Sucumbíos, Río Napo, Sacha Lodge, Pilchicocha, $0.472^{\circ} \mathrm{S}, 76.459^{\circ} \mathrm{W}, 228 \mathrm{~m}, 22$ February - 4 March 1994 (P Hibbs)(SEMC: ADP006174, male), 14-24 March 1994 (P Hibbs) (SEMC: ADP006173, female), 13-23 April 1994 (P Hibbs)(SEMC: ADP006132, female), 24 March - 3 June 1994 (P Hibbs)(SEMC: ADP007645, female). French GuIana, Cayenne, Foret de Maya, Commune Macouria, $4.9535^{\circ} \mathrm{S}, 52.4566^{\circ} \mathrm{W}, 32 \mathrm{~m}, 19$ December 2016 (S Brule, PH Dalens, E Poirier)(NMNH: ADP148976, male, Mitaraka, Commune Maripasoula, 2.2723ㅇ, 54.5152 ${ }^{\circ} \mathrm{W}, 445 \mathrm{~m}, 4$ March 2016 (S Brule, PH Dalens, E Poirier)(NMNH: ADP148975, female), Commune de Roura, Montagne des Chevaux, $4.7127^{\circ} \mathrm{N}, 52.3966^{\circ} \mathrm{W}, 90 \mathrm{~m}, 19$ June 2010 (S Brule, PH Dalens, E Poirier)(NMNH: ADP135774, male, ADP135788, female), 2 January 2011 (S Brule, PH Dalens, E Poirier)(NMNH: ADP130786, female), 28 March 2011 (S Brule, PH Dalens, E Poirier)(NMNH: ADP135814, male), 14 August 2011 (S Brule, PH Dalens, E Poirier)(NMNH: ADP135802, female), 21 August 2011 (S Brule, PH Dalens, E Poirier)(NMNH: ADP135820, female), 11 September 2011 (S Brule, PH Dalens, E Poirier)(NMNH: ADP135776, ADP135824, females), 25 September 2011 (S Brule, PH Dalens, E Poirier)(NMNH: ADP135770, female), 8 October 2011 (S Brule, PH Dalens, E Poirier)(NMNH: ADP135816, female), 23 October 2011 (S Brule, PH Dalens, E Poirier)(NMNH: ADP140522, male, ADP135772, female), 30 October 2011 (S Brule, PH Dalens, E Poirier)(NMNH: ADP135796, female), 12 November 2011 (S Brule, PH Dalens, E Poirier)(NMNH: ADP135780, female), 11 December 2011 (S Brule, PH Dalens, E Poirier)(NMNH: ADP135830, female), 18 December 2011 (S Brule, PH Dalens, E Poirier)(NMNH: ADP135800, ADP135810, females), 21 December 2011 (S Brule, PH Dalens, E Poirier)(NMNH: ADP135768, ADP135808, females), 24 December 2011 (S Brule, PH Dalens, E Poirier)(NMNH: 
ADP135798, female), 18 February 2012 (S Brule, PH Dalens, E Poirier)(NMNH: ADP128634, female), 3 November 2012 (S Brule, PH Dalens, E Poirier)(NMNH: ADP135828, male), 3 January 2013 (S Brule, PH Dalens, E Poirier)(NMNH: ADP135822, male), 27 January 2013 (S Brule, PH Dalens, E Poirier)(NMNH: ADP135811, male), 31 March 2013 (S Brule, PH Dalens, E Poirier)(NMNH: ADP135812, female), 20 April 2013 (S Brule, PH Dalens, E Poirier)(NMNH: ADP148093, male), 12 April 2014 (S Brule, PH Dalens, E Poirier)(NMNH: ADP140520, male), 19 July 2014 (S Brule, PH Dalens, E Poirier)(NMNH: ADP140531, male), 27 December 2014 (S Brule, PH Dalens, E Poirier)(NMNH: ADP148092, male), 3 January 2015 (S Brule, PH Dalens, E Poirier)(NMNH: ADP148091, male), 17 October 2015 (S Brule, PH Dalens, E Poirier)(NMNH: ADP148094, male), 2 January 2016 (S Brule, PH Dalens, E Poirier)(NMNH: ADP148095, ADP148096, ADP148097, males), 9 January 2016 (S Brule, PH Dalens, E Poirier)(NMNH: ADP148102, male), 30 April 2016 (S Brule, PH Dalens, E Poirier) (NMNH: ADP148871, male), Commune Matoury, Mont Grand, $4.862^{\circ} \mathrm{N}, 52.355^{\circ} \mathrm{W}$, 215m, 17 December 2012 (S Brule, PH Dalens, E Poirier)(NMNH: ADP135801, female), Inselberg, Nouragues, Commune de Regina, Saut Parare, $4.0334^{\circ} \mathrm{N}$, $52.6786^{\circ} \mathrm{W}, 51 \mathrm{~m}, 13$ October 2010 (S Brule, PH Dalens, E Poirier)(NMNH: ADP128636, female), Camp Inselberg, Nouragues, Commune de Regina, $4.0839^{\circ} \mathrm{N}$, $52.6813^{\circ} \mathrm{W}, 411 \mathrm{~m}, 22$ November 2012 (S Brule, PH Dalens, E Poirier)(NMNH: ADP147735, female), Region de Saul, Commune de Saul, Belvedere de Saul (point de vue), $3.6223^{\circ} \mathrm{N}, 53.2159^{\circ} \mathrm{W}, 283-325 \mathrm{~m}, 5$ February 2010 (S Brule, PH Dalens, E Poirier)(NMNH: ADP130781, female), 5 February 2010 (S Brule, PH Dalens, E Poirier)(NMNH: ADP130782, female), 20 December 2010 (S Brule, PH Dalens, E Poirier)(NMNH: ADP130784, female), 24 January 2011 (S Brule, PH Dalens, E Poirier) (NMNH: ADP130783, female), 4 February 2011 (S Brule, PH Dalens, E Poirier) (NMNH: ADP134146, female), 7 February 2011 (S Brule, PH Dalens, E Poirier) (NMNH: ADP130785, ADP134155, females), 22 March 2011 (S Brule, PH Dalens, E Poirier)(NMNH: ADP134142, ADP134150, males, ADP134148, ADP134149, females), 30 March 2011 (S Brule, PH Dalens, E Poirier)(NMNH: ADP134147, male), 15 June 2011 (S Brule, PH Dalens, E Poirier)(NMNH: ADP134296, female), 11 August 2011 (S Brule, PH Dalens, E Poirier)(NMNH: ADP134154, female), 13 December 2011 (S Brule, PH Dalens, E Poirier)(NMNH: ADP134144, female), 3 October 2012 (S Brule, PH Dalens, E Poirier)(NMNH: ADP134298, male), 11 December 2012 (S Brule, PH Dalens, E Poirier)(NMNH: ADP134153, male, ADP134151, ADP134152, females), 16 January 2013 (S Brule, PH Dalens, E Poirier)(NMNH: ADP134145, female), 27 January 2013 (S Brule, PH Dalens, E Poirier)(NMNH: ADP147733, female), Foret de Maya, Commune Macouria, $4.9552^{\circ} \mathrm{N}, 52.4603^{\circ} \mathrm{W}$, 30m, 19 December 2016 (S Brule, PH Dalens, E Poirier)(NMNH: ADP151236, male). Perú, Loreto, Pacaya-Samiria National Reserve, Río Samiria, Cocha Shinguito, $5.1775^{\circ} \mathrm{S}, 76.6556^{\circ} \mathrm{W}, 112 \mathrm{~m}, 29$ August 1991 (TL Erwin, MG Pogue)(NMNH: ADP051385, ADP051413, males, ADP051412, ADP051415, females), 19 June 1990 (TL Erwin, et al.)(NMNH: ADP094114, ADP094121, males, ADP094067, female), 
Río Samiria (South Branch), Camp Terry, 5.6951으, 75.2243W, 129m, 14 May 1990 (TL Erwin, et al.)(NMNH: ADP007644, female), 16 May 1990 (TL Erwin)(NMNH: ADP094121, ADP094084, females); Madre de Dios, Manu Reserved Zone, Río Manu, BIOLAT Biological Station, Pakitza, $11.9446^{\circ} \mathrm{S}, 71.2831^{\circ} \mathrm{W}, 356 \mathrm{~m}, 14$ Octo- $^{\circ}$ ber 1991 (TL Erwin, MG Pogue)(NMNH: ADP007512, ADP007513, females), 16 October 1991 (TL Erwin, MG Pogue)(NMNH: ADP007530, female), 23 June 1993 (TL Erwin, F. Pfuno)(NMNH: ADP007531, male, ADP007529, ADP007551, females), 28 September 1991 (TL Erwin, MG Pogue)(NMNH: ADP007528, female), 6 October 1991 (TL Erwin, MG Pogue)(NMNH: ADP007511, female), Reserva Nacional Tambopata, $30 \mathrm{~km}$ (air) SW Puerto Maldonado, Explorer's Inn, $12.8364^{\circ} \mathrm{S}$, 69.2936 W, 209m, 16 March 1982 (TL Erwin, et al.)(NMNH: ADP007642, male), 3 October - 15 November 1983, NE Stork, et al.)(NMNH: ADP135873, female), 2 March 1984 (TL Erwin, et al.)(NMNH: ADP007643, male), 8 September 1984 (TL Erwin, et al.)(NMNH: ADP007641, female).Venezuela, Amazonas, Cerro de la Neblina, Rio Baria Basecamp, 0.837 N, 66.162 W, 138m, 10-20 February 1985 (PJ Spangler, PM Spangler, et al.)(NMNH: ADP006177, female), 20 February 1985 (PJ Spangler, PM Spangler, et al.)(NMNH: ADP006176, female), 21-28 February 1985 (PJ Spangler, PM Spangler, et al.)(NMNH: ADP005803, male).

Geographic distribution (Fig. 11). This species is currently known from the type locality at Tefé, Brazil, and from Brazil - Amazonas, Pará, Rondonia; Ecuador, French Guiana, Perú, and Venezuela.

Notes. Reichardt (1973) reported the following additional specimens that we did not see: BrazIL - Amazonas: Itaituba (3 exs. MNHP); Manaus (1 ex. MZSP); Matuxadi, alto Rio Cauaburi (1 ex. MZSP): Tefé (4 exs. MNHP). Pará: Tapajos (2 exs. MNHP). Lorenz $(1998,2005)$ failed to record this species.

\section{Hyboptera lucida Henry \& Erwin, sp. n.} http://zoobank.org/BC3B2B01-C53D-48A6-B2B1-342CA9D2E53A

Pied humps-backed beetle

Figs 7B, 8, 10C, 11

Holotype. Female. French Guiana, Cayenne, Commune de Roura, Montagne des

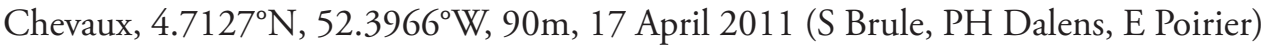
(NMNH: ADP128638).

Specific epithet. The epithet "lucida" is a Latinized singular feminine adjective of lucid, for clear, referring to the translucent patch near the apex of the elytron on adult members of this species.

Proposed english vernacular name. Pied humps-backed beetle.

Diagnosis. With the attributes of the genus and tuberculata species group as described above and adults with only dark non-metallic markings on the pronotal disc; elytra black with 4 small pale medio-apical tubercles. Venter completely piceous. Largest adults in the genus. 


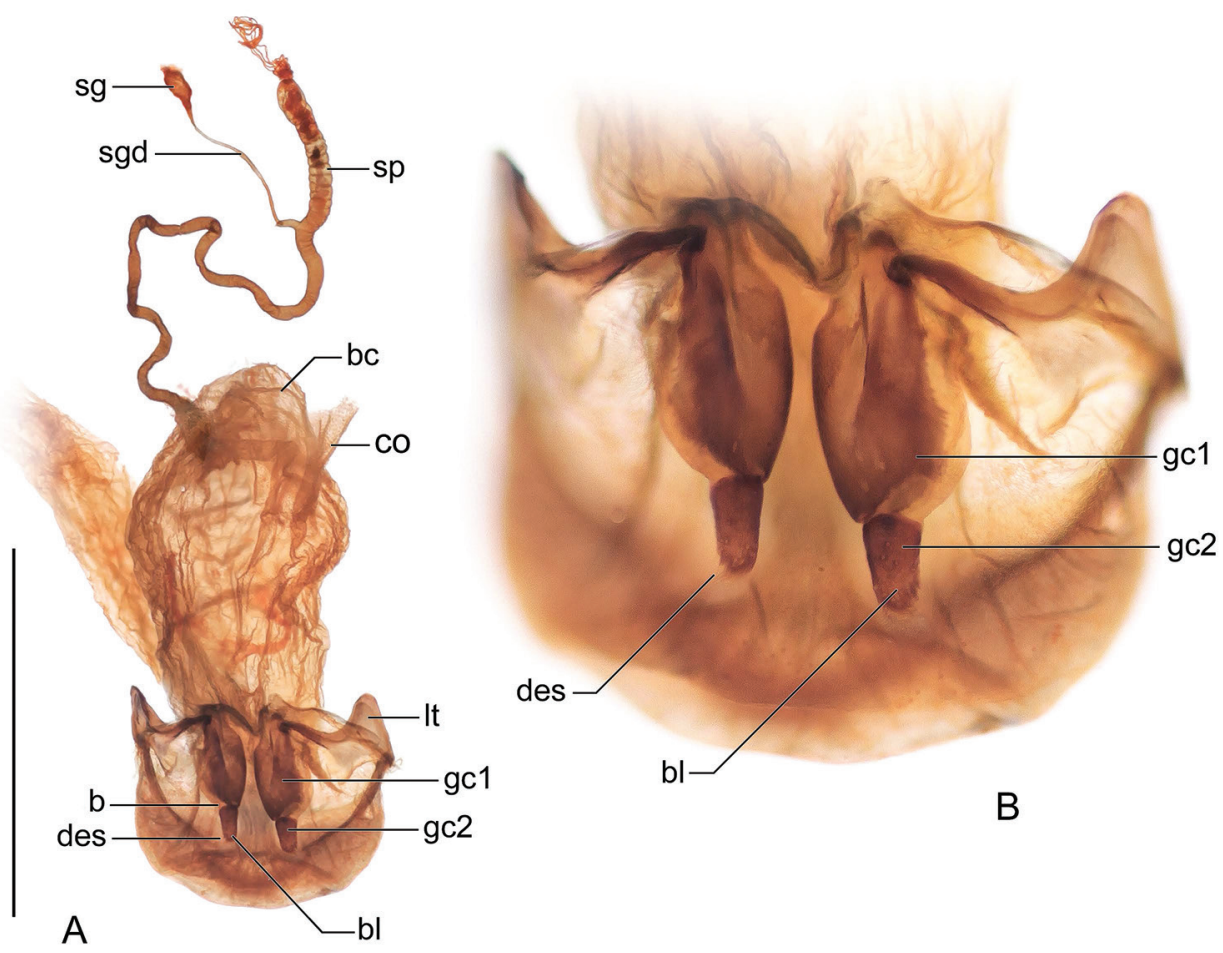

Figure 8. Digital Photo-illustration, female reproductive system dorsal and ventral aspects. Hyboptera lucida Henry \& Erwin sp. n., ADP148100. Legend, bc, bursa copulatrix; sg, spermathecal gland; sgd, spermathecal gland duct; sp, spermatheca. Dorsal aspect; lt, laterotergite; gc1, gonocoxite 1; gc2, gonocoxite 2, des, dorsal ensiform seta, b, base of gonocoxite 2; bl, blade of gonocoxite 2 . Scale line $=0.25 \mathrm{~mm}$.

Description. (Figs 7B, 8, 10C). Size: See Appendix 1. Length (SBL) long for genus, $\mathrm{ABL}=5.51-6.67 \mathrm{~mm}, \mathrm{SBL}=4.38-5.27 \mathrm{~mm}, \mathrm{TW}=2.36-3.25 \mathrm{~mm}$.

Color: See diagnosis, above.

Luster: Without any metallic highlights, matte.

Microsculpture: Mostly isodiametric and stretched, well-impressed, cells somewhat more stretched around elytral tubercles.

Head: Rugae moderately coarse, mostly without patterned arrangement. Eye very large, hemispheric, evenly rounded. Antenna short, barely reaching humerus. Labrum rectangulate, shallowly bilobed, anterior margin slightly emarginate. Neck coarsely transversely rugose.

Prothorax: Pronotum markedly broad, disc centrally shallowly depressed with coarse angulate rugae. Lateral margins broadly explanate and evenly rounded to level of lateral seta then straight to obtuse hind angle, base medially produced and rounded posteriorly.

Pterothorax: Normal for Agrina, fully winged. Elytron intervals 3 with 10 and interval 5 with 7 prominent discal unisetiferous tubercles, interval 4 with sub-apical pale colored "lens," other intervals moderately convex, side margin broadly explanate 
laterally only at middle third. Elytron broad and moderately short, much narrower in width than that of the pronotum at the broadest part, apex truncate, slightly rounded with distal corner broadly and obtusely rounded, disc not significantly convex, basal third slightly depressed. All interneurs well-impressed.

Legs: Femur dorso-ventrally moderately depressed, tibia coequal in length, more depressed; tarsus less than half the length of the tibia, fourth tarsomere markedly bilobed and with tarsal pad of setae.

Abdomen: Sparsely setiferous; normal ambulatory setae on sterna 3-5; female with two pairs of ambulatory setae on sternum 6 , medial pair of setae less than the length of lateral pair; males with only the lateral pair of longer setae.

Male genitalia: Phallus (Fig. 10C) with ostium of $1 / 6$ its length, catopic, apex very short, narrowly pointed, broadly rounded in dorsal aspect; endophallus with flagellum (obvious in illustration), flagellum not barbed. Parameres asymmetric, right very small, left larger.

Female genitalia: (Fig. 8). Ovipositor with broad triangular laterotergite (lt) and two robust gonocoxites (gc 1, gc 2); gonocoxite 1 apicolaterally not setose; gonocoxite 2 apically rounded, base (b) medium-size co-equal in width with blade (bl) which is short, blunt, with several dorsal ensiform setae (des), ventral ensiform seta absent, ensiform setae moderately short and robust; without ventral preapical nematiform setae. Reproductive tract proximally with moderately long, broad bursa copulatrix (bc), common oviduct (co) enters the bursa ventrally just anterior to bursal midlength, and long narrow corregated spermatheca (sp) distal to villous canal; spermathecal gland small, cylindrical; spermathecal gland duct (sgd) very narrow, attached to oviduct at base of its broadened portion.

Dispersal potential. These beetles are macropterous and capable of flight. They are moderately swift and agile runners. Adults of this species are attracted to light traps, and have been collected in FIT, Malaise, and SLAM traps.

Way of life. Adults are found in January-March, May-December, in all seasons, in lowlands (13-325 m.a.s.l.) in the lowland forests of Guyane.

Other specimens examined. French Guiana, Cayenne, Commune de Roura, Montagne des Chevaux, $4.7127^{\circ} \mathrm{N}, 52.3966^{\circ} \mathrm{W}, 90 \mathrm{~m}, 16$ July 2011 (S Brule, PH Dalens, E Poirier)(NMNH: ADP135813, male paratype), 2 January 2016 (S Brule, PH Dalens, E Poirier)(NMNH: ADP148100, female paratype), 26 March 2016 (S Brule, PH Dalens, E Poirier)(NMNH: ADP148222, male paratype), 20 May 2015 (S Brule, PH Dalens, E Poirier)(NMNH: ADP148099, male paratype), 23 October 2011 (S Brule, PH Dalens, E Poirier)(NMNH: ADP135793, female paratype), 23 September 2011 (S Brule, PH Dalens, E Poirier)(NMNH: ADP135785, male paratype), 25 September 2011 (S Brule, PH Dalens, E Poirier)(NMNH: ADP135825, female paratype), 31 March 2013 (S Brule, PH Dalens, E Poirier)(NMNH: ADP135807, male paratype), 4 February 2013 (S Brule, PH Dalens, E Poirier)(NMNH: ADP135831, male paratype), 16 April 2016 (S Brule, PH Dalens, E Poirier)(NMNH: ADP152487, female paratype), Commune Matoury, La Desiree, $4.8449^{\circ} \mathrm{N}, 52.3484^{\circ} \mathrm{W}, 20 \mathrm{~m}, 27$ September 2014 (S Brule, PH Dalens, E Poirier)(NMNH: ADP148098, female para- 
type), Trou Poisson, $5.4206^{\circ} \mathrm{N}, 53.0716^{\circ} \mathrm{W}, 13 \mathrm{~m}, 7$ May 2014 (S Brule, PH Dalens, E Poirier)(NMNH: ADP135783, female paratype), 7 May 2014 (S Brule, PH Dalens, E Poirier)(NMNH: ADP135829, female paratype), Inselberg Nouragues, Commune de Regina, Saut Parare, $4.0334^{\circ} \mathrm{N}, 52.6786^{\circ} \mathrm{W}, 51 \mathrm{~m}, 30$ September 2009 (S Brule, PH Dalens, E Poirier)(NMNH: ADP126231, female paratype), Region de Saul, Commune de Saul, Belvedere de Saul (point de vue), 3.6223 $\mathrm{N}, 53.2159^{\circ} \mathrm{W}, 283-325 \mathrm{~m}, 11$ December 2012 (S Brule, PH Dalens, E Poirier)(NMNH: ADP134135, female paratype), 20 December 2010 (S Brule, PH Dalens, E Poirier)(NMNH: ADP130776, female paratype), 21 June 2011 (S Brule, PH Dalens, E Poirier)(NMNH: ADP134134, female paratype), Cirque Orfion, Orapu RN2 PK65, Commune de Regina, $4.4962^{\circ} \mathrm{N}$, $52.3454^{\circ} \mathrm{W}, 81 \mathrm{~m}, 17$ September 2016 (S Brule, PH Dalens, E Poirier)(NMNH: ADP151235, male paratype).

Geographic distribution (Fig. 11). This species is currently known from the type locality in French Guiana and nearby areas.

\section{Hyboptera tuberculata (Dejean), 1825}

Tuberculate humps-backed beetle

Figs 9A, 10D, 11

\section{Lebia tuberculata Dejean, 1825: 272.}

Cryptobatis tuberculata; Gemminger and Harold (1868: 135).

Hyboptera tuberculata (Dejean); Chaudoir (1872: 162).

Aspasia verrucosa Reiche; Reichardt (1973: 53).

Lectotype. Here designated. Male. French Guiana, Cayenne, (MNHP).

Derivation of specific epithet. The species epithet "tuberculata" is a Latinized singular feminine adjective, referring to the bumpy attributes of the elytra.

Proposed English vernacular name. Tuberculate humps-backed beetle.

Diagnosis. With the attributes of the genus and tuberculata species group as described above and adults with only dark non-metallic markings on the pronotal disc, elytron just posterior to scutellum with only the suture pale in color, otherwise markedly infuscate.

Description. (Figs 9A, 10D). Size: See Appendix 1. Length (SBL) long for genus, $\mathrm{ABL}=4.32-5.88 \mathrm{~mm}, \mathrm{SBL}=3.94-4.85 \mathrm{~mm}, \mathrm{TW}=2.18-3.12 \mathrm{~mm}$.

Color: See diagnosis, above.

Luster: Without any metallic highlights, shiny.

Microsculpture: Mostly isodiametric and stretched, shallowly impressed, cells somewhat more stretched around elytral tubercles.

Head: Rugae moderately coarse, mostly chaotic. Eye markedly large, sub-hemispheric, evenly rounded anteriorly, subtly more prolonged posteriorly. Antenna short, barely reaching humerus. Labrum rectangulate, shallowly bilobed, anterior margin slightly emarginate. Neck transversely finely rugose. 


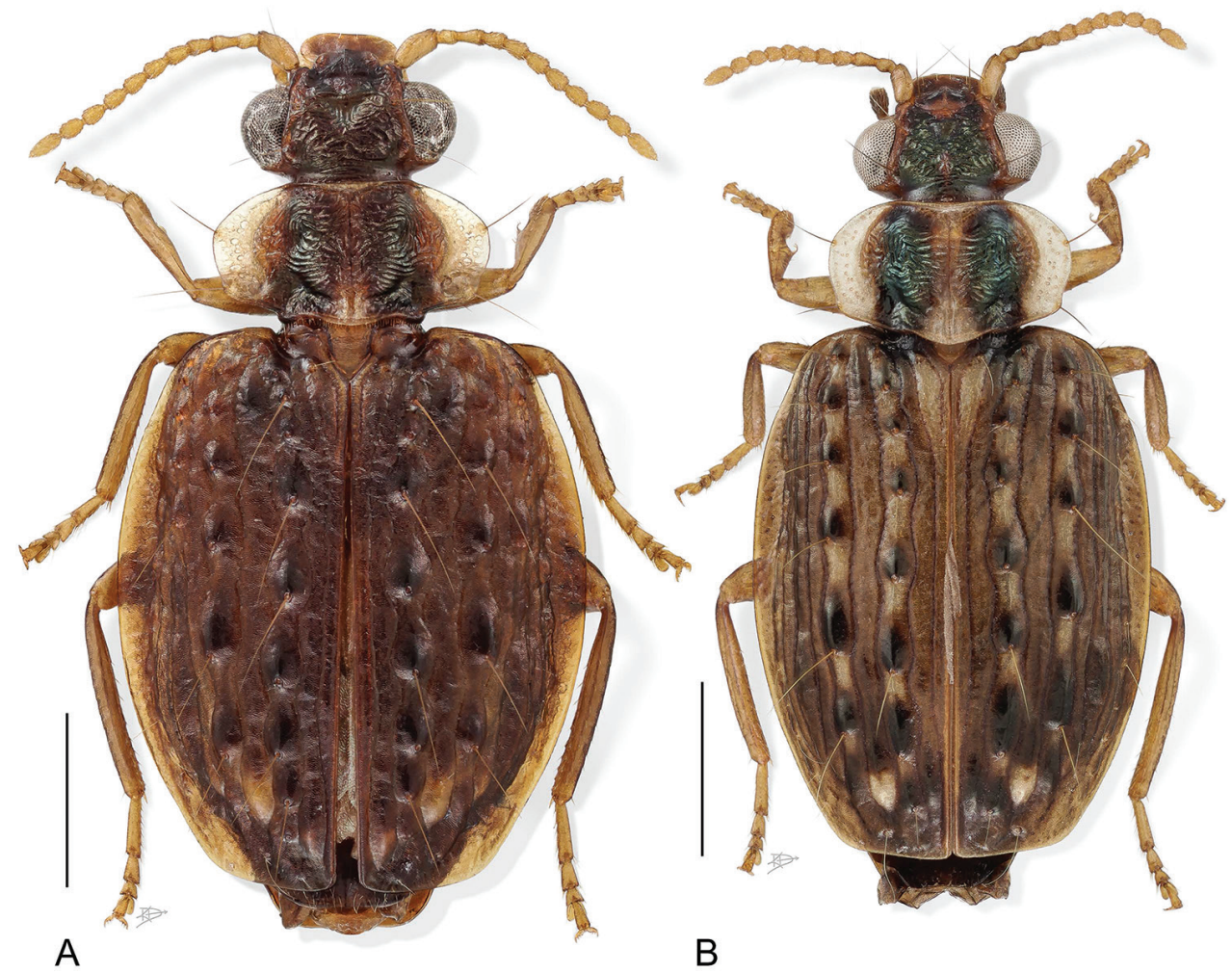

Figure 9. Digital Photo-illustration. Habitus, dorsal aspect. A Hyboptera tuberculata (Dejean), female, ADP007533 B Hyboptera verrucosa (Reiche), female, ADP086946.

Prothorax: Pronotum markedly broad, disc centrally moderately depressed with coarse angulate rugae. Lateral margins broadly explanate and evenly rounded then straight to obtuse hind angle, base medially produced and rounded posteriorly.

Pterothorax: Normal for Agrina, fully winged. Elytron intervals 3 with 8(9) and interval 5 with 6(7) prominent discal unisetiferous tubercles, interval 4 and 6 with subapical pale colored "lens," other intervals moderately convex, side margin broadly explanate laterally only at middle third. Elytron broad and moderately short, moderately narrower in width than that of the pronotum at the broadest part, apex truncate, slightly rounded with distal corner broadly and obtusely rounded, disc not significantly convex, basal third slightly depressed. All interneurs well-impressed.

Legs: Femur dorso-ventrally moderately depressed, tibia coequal in length, more depressed; tarsus less than half the length of the tibia, fourth tarsomere markedly bilobed and with tarsal pad of setae.

Abdomen: Sparsely setiferous; normal ambulatory setae on sterna 3-5; female with two pairs of ambulatory setae on sternum 6 , medial pair of setae less than the length of lateral pair; males with only the outer pair of longer setae. 

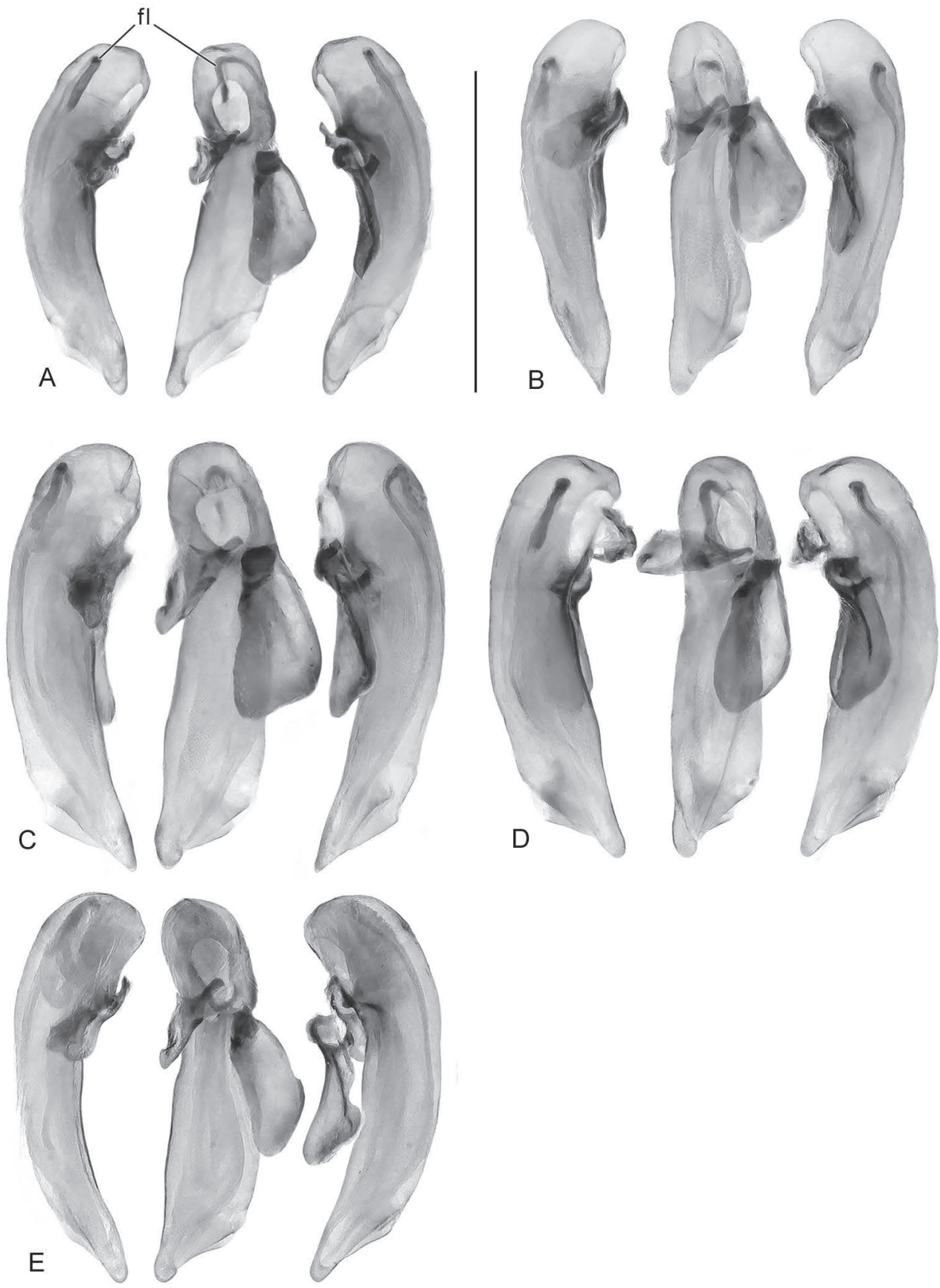

Figure 10. Digital Photo-illustration, male aedeagus in repose, dorsal, ventral, left lateral aspects: A $H y$ boptera apollonia Erwin, ADP100266 B Hyboptera dilutior Oberthür, ADP134143 C Hyboptera lucida Henry \& Erwin, sp. n., ADP135785 D Hyboptera tuberculata (Dejean), ADP135797 E Hyboptera verrucosa (Reiche), ADP082523. Legend, fl, flagellum. Scale line $=0.25 \mathrm{~mm}$. 


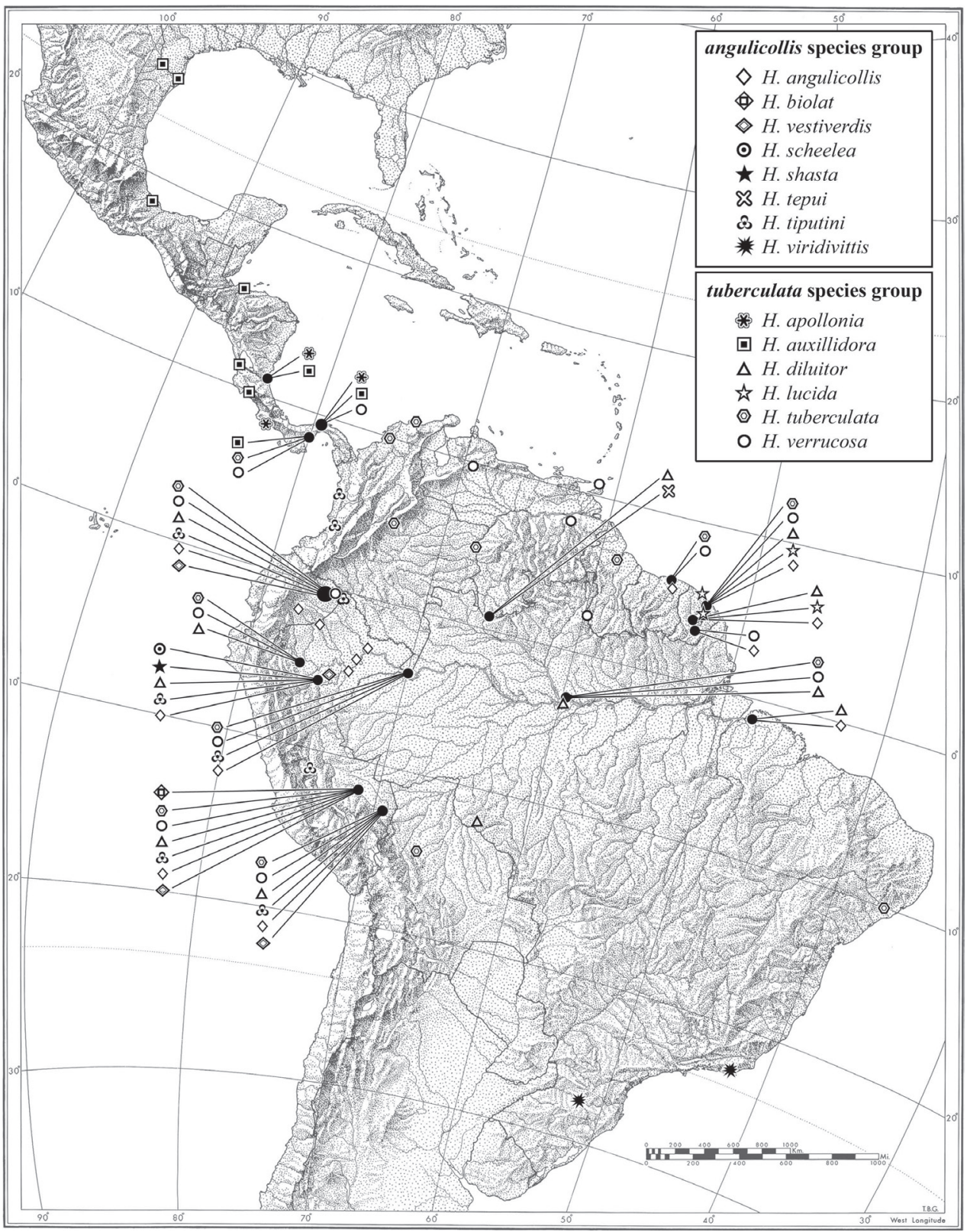

Figure I I. Distribution map for known localities of Hyboptera species.

Male genitalia: Phallus (Fig. 10D) robust with ostium of 1/6 its length, catopic, apex very short, broadly rounded in dorsal and lateral aspects; endophallus with flagellum (obvious in illustration), flagellum not barbed. Parameres asymmetric, right very small, left larger.

Female genitalia: Not investigated, likely similar to that of $H$. lucida (Fig. 8). 
Dispersal potential. These beetles are macropterous and capable of flight. They are moderately swift and agile runners. Adults of this species are attracted to C.D.C. Light traps.

Way of life. Adults are common in the lowlands and lower midlands (7-914 m a.s.l.) and appear to be generalists in a variety of rainforest biotopes including terra firme, dry and humid tropical forests, in gallery forests, and savanna forest patches. In these forests, they are commonly found in big trees with vines and epiphytes, in suspended medium to large-sized dry leaves, and in dense vine tangle plus leaves with light bamboo occurrence. Individuals can be found from January to December, in both the rainy and dry seasons. Member of this species have been recorded from the canopy of the following tree species using insecticidal fogging techniques: Pouteria reticulata ssp. reticulata cf.; Sloanea cordia; Browneopsis ucayalina; Talisia bitter; Mouriri guapira; Trichilia solitudinis; Eriotheca globosa cf.; Sloanea 1; Trichilia solitudinis; Simira cordifolia/rubescens cf.; Sarcaulus brasiliensis aff. "burnt"; Catatola costaricana aff.; Pourouma mollis ssp. triloba cf.; Alchornea triplinervia cf.; Duguetia surinamensis cf.; Casearia avitensis cf.; Nectandra crassiloba cf.; Trichilia rubra cf.; Coccoloba densifrons cf. traditional; Iriartea deltoidea; Micropholis venulosa cf.; Sagotia racemosa; Virola obovata; Pouteria baehniana cf.; Pourouma bicolor ssp. bicolor cf.; Inga bourgonii cf.; Garcinia macrophylla cf.; Chrysobalanaceae surreptitious; Oenocarpus bataua; Neea divetuberculate; Semaphyllanthe megistocaula cf.; Lauraceae redvein.

Other specimens examined. Bolivia, Beni, $53 \mathrm{~km}$ E San Borja, Estación Biológica Beni, Palm Camp, $14.8676^{\circ} \mathrm{S}, 66.3265^{\circ} \mathrm{W}, 177 \mathrm{~m}, 25-30$ July 1988 (TL Erwin)(NMNH: ADP005759, male, ADP135777, female). BraziL, Amazonas, north of Manaus, on Amazonas 010 at $\mathrm{Km}$ 26, Reserva Ducke, $2.918^{\circ} \mathrm{S}, 59.971^{\circ} \mathrm{W}, 70 \mathrm{~m}$, 27 October 1977 (J Arias)(NMNH: ADP005758, female), 8 August 1978 (J Arias)(NMNH: ADP135819, female); Sergipe, Estação Ecológica da Serra de Itabaiana, $10.7297^{\circ} \mathrm{S}, 37.3064^{\circ} \mathrm{W}, 166 \mathrm{~m}, 14-20$ September 1999 (A Bonaldo)(NMNH: MCM166.415, ADP144921, male). Colombia, Amazonas, PNN Amacayacu, Mocagua, 3.84 ${ }^{\circ} \mathrm{S}, 70.22^{\circ} \mathrm{W}, 76 \mathrm{~m}, 3-9$ April 2000 (A Parente)(IAvH: IAvH-E-3063, ADP145177, male)(IAvH: IAvH-E-10944, ADP145183, female), 6-12 June 2000 (A Parente)(IAvH: IAvH-E-73787, ADP145145, male), 12-19 June 2000 (A Parente) (IAvH: IAvH-E-3056, ADP145193)(IAvH: IAvH-E-3093, ADP145203)(IAvH: IAvH-E-3095, ADP145173, males)(IAvH: IAvH-E-3096, ADP145187, female), 26 June - 6 July 2000 (A Parente)(IAvH: IAvH-E-73786, ADP145175, male), 7-19 July 2000 (A Parente)(IAvH: IAvH-E-40755, ADP145171)(IAvH: IAvH-E-73785, ADP145169, males)(IAvH: IAvH-E-73775, ADP145181, female); BolIvar, Zambrano, Hda. Monterrey - Rio Magdalena, $9.6293^{\circ} \mathrm{N}, 74.9123^{\circ} \mathrm{W}, 70 \mathrm{~m}, 16-30$ August 1993 (F Fernanadez, G Ulloa)(IAvH: IAvH-E-2449, ADP145154, female), September 1993 (F Fernanadez, G Ulloa)(IAvH: IAvH-E-73780, ADP145186, male), 8 October 1993 (G Ulloa, F Fernanadez)(IAvH: IAvH-E-2449, ADP145182, female), SFF Los Colorados, Diana, $9.90^{\circ} \mathrm{N}, 75.11^{\circ} \mathrm{W}, 237 \mathrm{~m}, 2-16$ January 2001 (E Deulufeut)(IAvH: IAvH-E-2320, ADP145194)(IAvH: IAvH-E-73779, ADP145180, males)(IAvH: IAvH-E-73778, ADP145196, female), La Suiris, $9.900^{\circ} \mathrm{N}, 75.1154^{\circ} \mathrm{W}, 236 \mathrm{~m}, 1-15$ 
September 2001 (E Deulufeut)(IAvH: IAvH-E-40756, ADP145184, male), SFF Los Colorados Venado, $9.900^{\circ} \mathrm{N}, 75.1153^{\circ} \mathrm{W}, 235 \mathrm{~m}, 2-17$ October 2000 (E Deulufeut) (IAvH: IAvH-E-73776, ADP145190)(IAvH: IAvH-E-73777, ADP145188, females); Casanare, Aguazul, $5.18^{\circ} \mathrm{N}, 72.55^{\circ} \mathrm{W}, 701 \mathrm{~m}, 22$ September 1995 (F Fernanadez) (IAvH: IAvH-E-2450, ADP145152, female); Magdelena, PNN Tayrona, Canaveral, $11.342^{\circ} \mathrm{N}, 074.031^{\circ} \mathrm{W}, 25 \mathrm{~m}, 17$ October -3 November 2000 (R Henriquez) (IAvH: IAvH-E-73781, ADP145178, male)(IAvH: IAvH-E-73782, ADP145174) (IAvH: IAvH-E-73783, ADP145170, females), 4-15 December 2000, R Henriquez) (IAvH: IAvH-E-3532, ADP145176)(IAvH: IAvH-E-73784, ADP145166, females), Vichada, PNN Tuparro, $5.36^{\circ} \mathrm{N}, 67.84^{\circ} \mathrm{W}, 71 \mathrm{~m}, 8-14$ December 2000 (W Villalba) (IAvH: IAvH-E-2741, ADP145148, male). Ecuador, Orellana, Reserva Ethnica Huaorani, 39 km S Pompeya, Estación Científica Yasuní - Onkone Gare Camp, Erwin Piraña Plot, transect 10 , station 2, $0.6590^{\circ} \mathrm{S}, 76.453^{\circ} \mathrm{W}, 220-250 \mathrm{~m}, 8$ October 1995 (TL Erwin, et al.)(NMNH: ADP137752, female paratype), Erwin Piraña Plot, transect 10, station 5, $0.6540^{\circ} \mathrm{S}, 76.453^{\circ} \mathrm{W}, 220-250 \mathrm{~m}, 4$ October 1996 (TL Erwin, et al.)(NMNH: ADP137369, male paratype), Erwin Pirańa Plot, transect 10, station 9, $0.6540^{\circ} \mathrm{S}, 76.453^{\circ} \mathrm{W}, 220-250 \mathrm{~m}, 23$ January 2006 (TL Erwin, et al.)(NMNH: ADP133868, male paratype), Erwin Piraña Plot, transect 4, station 4, $0.6570^{\circ} \mathrm{S}$, $76.498^{\circ} \mathrm{W}, 220-250 \mathrm{~m}, 3$ July 1995 (TL Erwin, et al.)(NMNH: ADP135805, male paratype), Erwin Pirańa Plot, transect 5, station 2, $0.6566^{\circ} \mathrm{S}, 76.490^{\circ} \mathrm{W}, 220-250 \mathrm{~m}$, 12 February 1995 (TL Erwin, et al.)(NMNH: ADP005762, female paratype), Erwin Piraña Plot, transect 5 , station $2,0.6566^{\circ} \mathrm{S}, 76.496^{\circ} \mathrm{W}, 220-250 \mathrm{~m}, 9$ October 1994 (TL Erwin, et al.)(NMNH: ADP138806, male paratype), Erwin Piraña Plot, transect 6 , station $1,0.6561^{\circ} \mathrm{S}, 76.483^{\circ} \mathrm{W}, 220-250 \mathrm{~m}, 7$ February 1996 (TL Erwin, et al.)(NMNH: ADP137383, male paratype), Erwin Piraña Plot, transect 6, station $1,0.6561^{\circ} \mathrm{S}, 76.485^{\circ} \mathrm{W}, 220-250 \mathrm{~m}, 29$ January 2006 (TL Erwin, et al.)(NMNH: ADP133852, female paratype), Erwin Piraña Plot, transect 6, station 4, $0.6561^{\circ} \mathrm{S}$, $76.4483^{\circ} \mathrm{W}, 220-250 \mathrm{~m}, 22$ June 1996 (TL Erwin, et al.)(NMNH: ADP137355, female paratype), Erwin Pirańa Plot, transect 6, station 6, $0.6561^{\circ} \mathrm{S}, 76.483^{\circ} \mathrm{W}, 220$ 250m, 22 July 1996 (TL Erwin, et al.)(NMNH: ADP137746, male paratype), Erwin Piraña Plot, transect 6, station 7, 0.6561 ${ }^{\circ} \mathrm{S}, 76.4483^{\circ} \mathrm{W}, 220-250 \mathrm{~m}, 2$ October 1996 (TL Erwin, et al.)(NMNH: ADP135891, male paratype), Erwin Pirańa Plot, transect 6 , station $8,0.6561^{\circ} \mathrm{S}, 76.4483^{\circ} \mathrm{W}, 220-250 \mathrm{~m}, 7$ February 1996 (TL Erwin, et al.)(NMNH: ADP137353, female paratype), Erwin Piraña Plot, transect 8, station 4, $0.6551^{\circ} \mathrm{S}, 76.4403^{\circ} \mathrm{W}, 220-250 \mathrm{~m}, 7$ October 1995 (TL Erwin, et al.)(NMNH: ADP135970, male paratype), Erwin Piraña Plot, transect 8, station 6, $0.6551^{\circ}$ S, $76.4483^{\circ} \mathrm{W}, 220-250 \mathrm{~m}, 7$ October 1995 (TL Erwin, et al.)(NMNH: ADP137760, female paratype); Yasuni National Park (edge), $95.43 \mathrm{~km}$ E (heading $101.46^{\circ}$ ) Coca, Tiputini Biodiversity Station, Erwin Harpia Plot: transect 4, station 8, $0.6316^{\circ} \mathrm{S}$, $76.1443^{\circ} \mathrm{W}, 208 \mathrm{~m}, 24$ October 1998 (TL Erwin, et al.)(NMNH: ADP135821, male), Erwin Harpia Plot: transect 8, station 4, 0.6278 ${ }^{\circ}$ S, 76.1443 ${ }^{\circ} \mathrm{W}, 203 \mathrm{~m}, 4$ July 1998 (TL Erwin, et al.)(NMNH: ADP135797, male). Yasuni National Park (edge), 95.43 $\mathrm{km}$ E (heading $101.46^{\circ}$ ) Coca, Tiputini Biodiversity Station, Erwin Harpia Plot, tran- 
sect 10 , station $6,0.6262^{\circ} \mathrm{S}, 76.1443^{\circ} \mathrm{W}, 214 \mathrm{~m}, 21$ October 1998 (TL Erwin, et al.) (NMNH: ADP135775, female paratype), Erwin Harpia Plot, transect 10, station 9, $0.6269^{\circ} \mathrm{S}, 76.1443^{\circ} \mathrm{W}, 214 \mathrm{~m}, 5$ July 1998 (TL Erwin, et al.)(NMNH: ADP135803, female paratype), Erwin Harpia Plot, transect 3, station $10,0.6332^{\circ} \mathrm{S}, 76.1443^{\circ} \mathrm{W}$, 207m, 8 February 1999 (TL Erwin, et al.)(NMNH: ADP135773, male paratype), Erwin Harpia Plot, transect 4, station 1, $0.6316^{\circ} \mathrm{S}, 76.1443^{\circ} \mathrm{W}, 208 \mathrm{~m}, 24$ October 1998 (TL Erwin, et al.)(NMNH: ADP135779, female paratype), Erwin Harpia Plot, transect 6, station $9,0.6295^{\circ} \mathrm{S}, 76.1443^{\circ} \mathrm{W}, 199 \mathrm{~m}, 7$ February 1999 (TL Erwin, et al.)(NMNH: ADP135835, female paratype), Erwin Harpia Plot, transect 7, station $1,0.6295^{\circ} \mathrm{S}, 76.1443^{\circ} \mathrm{W}, 203 \mathrm{~m}$, Erwin Harpia Plot: transect 4, station 4, $0.6316^{\circ} \mathrm{S}$, $76.1443^{\circ} \mathrm{W}, 208 \mathrm{~m}, 20$ February 2001 (TL Erwin, et al.)(NMNH: ADP139100, female), 29 September 2000 (TL Erwin, et al.)(NMNH: ADP139132, female paratype), Erwin Harpia Plot, transect 7, station 6, $0.6287^{\circ} \mathrm{S}, 76.1443^{\circ} \mathrm{W}, 203 \mathrm{~m}, 6$ February 1999 (TL Erwin, et al.)(NMNH: ADP135827, male paratype); Sucumbíos, Río Napo, Sacha Lodge, Pilchicocha, $0.472^{\circ} \mathrm{S}, 76.459^{\circ} \mathrm{W}, 228 \mathrm{~m}, 12-22$ February 1994 (P Hibbs)(SEMC: ADP005757, female paratype), 24 May - 3 June 1994 (P Hibbs) (SEMC: ADP005756, female paratype). French Guiana, Cayenne, Commune de Roura, Montagne des Chevaux, $4.7127^{\circ} \mathrm{N}, 52.3966^{\circ} \mathrm{W}, 90 \mathrm{~m}, 23$ January 2016 (S Brule, PH Dalens, E Poirier)(NMNH: ADP144920, male). Guyana, Bartica, $6.3970^{\circ} \mathrm{N}$, 58.6268 W, 20m, 12 May 1924 (AMNH: ADP005760, male). Panamá, Chiriqui, Volcan de Chiriqui, 610-914m, 26 May 1983 (GC Champion)(BMNH: ADP144995, male); Cocle, El Valle, $8.603^{\circ} \mathrm{N}, 80.152^{\circ} \mathrm{W}, 800 \mathrm{~m}, 25-28$ May 1983 (WE Steiner) (NMNH: ADP144994, male). Perú, Loreto, Pacaya-Samiria National Reserve, Río Samiria, Cocha Shinguito, 5.1775 $\mathrm{S}, 76.6556^{\circ} \mathrm{W}, 112 \mathrm{~m}, 22$ May 1990 (TL Erwin, et al.)(NMNH: ADP086940, male, ADP086960, female); Madre de Dios, Manu Reserved Zone, Río Manu, BIOLAT Biological Station, $11.9446^{\circ} \mathrm{S}, 71.2831^{\circ} \mathrm{W}$, 356m, August-September 1988 (TL Erwin)(NMNH: ADP007532, ADP007535, males, ADP007533, ADP007534, females), 7 September 1988 (TL Erwin)(NMNH: ADP007550, female), 21 September 1991 (TL Erwin)(NMNH: ADP007552, male), 30 September 1991 (TL Erwin, MG Pogue)(NMNH: ADP135823, male), 22 June 1993 (TL Erwin, F. Pfuno S)(NMNH: ADP007553, ADP007554, females), Reserva Nacional Tambopata, $30 \mathrm{~km}$ (air) SW Puerto Maldonado, Explorer's Inn, $12.8364^{\circ} \mathrm{S}$, 69.2936 $6^{\circ}$, 209m, 30 April 1984 (TL Erwin, et al.)(NMNH: ADP005754, male), 2 May 1984 (TL Erwin, et al.)(NMNH: ADP005755, female). Suriname, ParamarIво, Combe, $5.8427^{\circ} \mathrm{N}, 55.1600^{\circ} \mathrm{W}, 15 \mathrm{~m}, 6$ January 1956 (DC Geijskes)(NBCL: ADP005761, male); WANICA, Lelydorp, Sumatra weg, $5.700^{\circ} \mathrm{N}, 55.198^{\circ} \mathrm{W}, 7 \mathrm{~m}, 28$ November 1939 (DC Geijskes)(NBCL: ADP005805, male).

Geographic distribution (Fig. 11). This species is currently known from the type locality in French Guiana and nearby areas and from Bolivia, Brazil - (Amazonas, Sergipe), Colombia, Ecuador, Guyana, Perú, and Suriname.

Notes. Reichardt (1971) synonomized the following species with $H$. tuberculata Dejean. We do not agree with this based on the numerous attributes we studied and included in the descriptions. 


\section{Hyboptera verrucosa (Reiche), 1842, stat. rest.}

Verrucose humps-backed beetle

Figs 9B, 10E, 11

Aspasia verrucosa Reiche, 1842: 311.

Hyboptera verrucosa (Reiche); Chaudoir (1872: 164).

Lebia tuberculata Dejean; Reichardt (1971: 53).

Holotype. Sex unknown. Colombia ("Nouvelle-Grenade"), (MNHP). Type locality herewith restricted to Colombia, Amazonas, Leticia, $4.2242^{\circ} \mathrm{S}, 69.9449^{\circ} \mathrm{W}, 70$ m.s.a.l.

Derivation of specific epithet. The specific epithet, verrucosa, is a feminine Latin adjective referring to the tuberculate elytra.

Proposed English vernacular name. Verrucose humps-backed beetle.

Diagnosis. With the attributes of the genus and tuberculata species group as described above and adults with only dark non-metallic markings on the pronotal disc, pronoum with rounded margins, elytron just posterior to scutellum with a V-shaped pale area encompassing the scutellum and sutural and first interval, venter substantially infuscate, and elytra narrow, not broadly arcuate.

Description. (Figs 9B, 10E). Size: See Appendix 1. Length (SBL) long for genus, $\mathrm{ABL}=4.47-5.66 \mathrm{~mm}, \mathrm{SBL}=3.79-4.90 \mathrm{~mm}, \mathrm{TW}=2.06-2.85 \mathrm{~mm}$.

Color: See diagnosis, above.

Luster: Without any metallic highlights, shiny and matte.

Microsculpture: Mostly isodiametric and stretched, shallowly impressed, cells somewhat more stretched around elytral tubercles.

Head: Rugae moderately coarse, mostly without patterned arrangement. Eye markedly large, sub-hemispheric, evenly rounded anteriorly, subtly more prolonged posteriorly. Antenna short, barely reaching humerus. Labrum rectangulate, shallowly bilobed, anterior margin slightly emarginate. Neck finely transversely rugose.

Prothorax: Pronotum moderately broad, disc centrally depressed with coarse angulate rugae. Lateral margins broadly explanate and evenly rounded to obtuse hind angle, base medially produced and rounded posteriorly.

Pterothorax: Normal for Agrina, fully winged. Elytron intervals 3 with 8, and interval 5 with 6 prominent discal unisetiferous tubercles, interval 4 with sub-apical pale colored "lens," other intervals moderately convex, side margin broadly explanate laterally only at middle third. Elytron broad and moderately short, much narrower in width than that of the pronotum at the broadest part, apex truncate, slightly rounded with distal corner broadly and obtusely rounded, disc not significantly convex, basal third slightly depressed. All interneurs well-impressed.

Legs: Femur dorso-ventrally moderately depressed, tibia coequal in length, more depressed; tarsus less than half the length of the tibia, fourth tarsomere markedly bilobed and with tarsal pad of setae. 
Abdomen: Sparsely setiferous; normal ambulatory setae on sterna 3-5; female with two pairs of ambulatory setae on sternum 6 , medial pair of setae less than the length of lateral pair; males with only the lateral pair of longer setae.

Male genitalia: (Fig. 10E). With ostium of $1 / 5$ its length, catopic, apex short, rounded; endophallus with flagellum, flagellum not barbed. Parameres asymmetric, right very small, left larger.

Female genitalia: Not investigated, likely similar to that of H. lucida (Fig. 8).

Dispersal potential. These beetles are macropterous and capable of flight. They are moderately swift and agile runners. Adults of this species are attracted to C.D.C. light traps.

Way of life. Adults are common in the lowlands (6 to 829 m.a.s.l.) and appear to be generalists in rainforest biotopes such as terra firme and secondary rain/pine forest. In these forests, they are commonly found in big trees with vines and epiphytes, in suspended dry Cecropia leaves, and in dry Astrocaryum chambira Burret palm frond skirts. Individuals can be found in January-November, in both the rainy and dry seasons. Member of this species have been recorded from the canopy of the following tree species using insecticidal fogging techniques: Chrysophyllum argenteum cf.; Sterculia colombiana cf.; Parkia multijuga cf.; Naucleopsis herrerensis cf.; Matisia malacocalyx cf.; Pseudolmedia laevigata; Browneopsis ucayalina; Simira cordifolialrubescens cf.; Brownea grandiceps cf.; Talisia bitter; Virola decorticans cf.; Pouteria cuspidata ssp. robusta cf.; Diospyros sericea; Guarea silvatica; Lacistema nena cf.; Guatteria glaberrima cf.; Luehea seemanni; Cordia alliodora.

Other specimens examined. BraziL, Amazonas, km $60 \mathrm{~N}$ Manaus, $18.1 \mathrm{~km}$ W Campinas Field Station, $2.468^{\circ} \mathrm{N}, 60.156^{\circ} \mathrm{W}, 113 \mathrm{~m}, 22$ February 1979 (TL Erwin, et al.) (NMNH: ADP007595, male), north of Manaus, on Amazonas 010 at Km 26, Reserva Ducke, $2.918^{\circ} \mathrm{S}, 59.971^{\circ} \mathrm{W}, 70 \mathrm{~m}, 29$ November 1977 (J Arias)(NMNH: ADP007619, male). Colombia, Amazonas, Leticia, 4.2242 $\mathrm{S}, 69.9449^{\circ} \mathrm{W}, 70 \mathrm{~m}, 19-25$ February 1972 (HF Howden, A Howden)(NMNH: ADP007579, male). Ecuador, Orellana, Reserva Ethnica Huaorani, 39 km S, Pompeya, Estación Científica Yasuní - Onkone Gare Camp, Erwin Pirańa Plot: transect 2, station 6, 0.6581 ${ }^{\circ} \mathrm{S}, 76.4513^{\circ} \mathrm{W}, 220-250 \mathrm{~m}, 22$ January 1994 (TL Erwin, et al.)(NMNH: ADP138694, male), Erwin Pirańa Plot: transect 10, station $9,0.6540^{\circ} \mathrm{S}, 76.4453^{\circ} \mathrm{W}, 220-250 \mathrm{~m}, 23$ January 1994 (TL Erwin, et al.)(NMNH: ADP138588, male), Erwin Pirańa Plot: transect 8, station 3, $0.6551^{\circ} \mathrm{S}, 76.4403^{\circ} \mathrm{W}$, 220-250m, 24 January 1994 (TL Erwin, et al.)(NMNH: ADP138834, male), Erwin Piraña Plot: transect 7 , station $10,0.6556^{\circ} \mathrm{S}, 76.4474^{\circ} \mathrm{W}, 220-250 \mathrm{~m}, 7$ October 1994 (TL Erwin, et al.)(NMNH: ADP135867, female), Erwin Piraña Plot: transect 6, station $9,0.6561^{\circ} \mathrm{S}, 76.4483^{\circ} \mathrm{W}, 220-250 \mathrm{~m}, 12$ February 1995 (TL Erwin, et al.)(NMNH: ADP135871, male), Erwin Piraña Plot: transect 1, station 4, $0.6586^{\circ} \mathrm{S}, 76.4521^{\circ} \mathrm{W}, 220$ 250m, 4 October 1995 (TL Erwin, et al.)(NMNH: ADP135911, male), Erwin Piraña Plot: transect 6 , station $10,0.6561^{\circ} \mathrm{S}, 76.4483^{\circ} \mathrm{W}, 220-250 \mathrm{~m}, 2$ October 1996 (TL Erwin, et al.)(NMNH: ADP135924, female), Yasuni National Park (edge), $95.43 \mathrm{~km} \mathrm{E}$ (heading $101.46^{\circ}$ ), Coca, Tiputini Biodiversity Station, Erwin Harpia Plot: transect 6, station 1, $0.6295^{\circ} \mathrm{S}, 76.1443^{\circ} \mathrm{W}, 220-250 \mathrm{~m}, 26$ October 1998 (TL Erwin, et al.)(NMNH: ADP135869, female). French Guiana, Cayenne, Commune de Roura, Montagne des 
Chevaux, $4.7127^{\circ} \mathrm{N}, 52.3966^{\circ} \mathrm{W}, 90 \mathrm{~m}, 19$ October 2013 (S Brule, PH Dalens, E Poirier) (NMNH: ADP140530, male), 29 March 2014 (S Brule, PH Dalens, E Poirier)(NMNH: ADP140518, female), Region de Saul, Commune de Saul, Belvedere de Saul, 3.6223N, 53.2159 ${ }^{\circ} \mathrm{W}, 283-325 \mathrm{~m}, 23$ February 2011 (S Brule, PH Dalens, E Poirier)(NMNH: ADP134156, female), 17 October 2012 (S Brule, PH Dalens, E Poirier)(NMNH: ADP134297, male), Foret de Maya, Commune Macouria, $4.9552^{\circ} \mathrm{N}, 52.4603^{\circ} \mathrm{W}, 30 \mathrm{~m}$, 19 December 2016 (S Brule, PH Dalens, E Poirier)(NMNH: ADP151234, female), Cirque Orfion, Orapu RN2 PK65, Commune de Regina, $4.4962^{\circ} \mathrm{N}, 52.3454^{\circ} \mathrm{W}, 81 \mathrm{~m}$, 24 September 2016 (S Brule, PH Dalens, E Poirier)(NMNH: ADP151233, male). PaNamá, Canal Zone, Ancón, 8.9588 $\mathrm{N}, 79.5541^{\circ} \mathrm{W}, 36 \mathrm{~m}, 5$ November 1921 (H Osborn) (NMNH: ADP058099, female), Lion Hill Island, $9.2266^{\circ} \mathrm{N}, 79.8916^{\circ} \mathrm{W}, 68 \mathrm{~m}, 29$ June

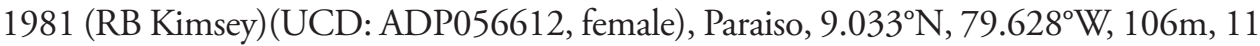
January 1911 (EA Schwarz)(NMNH: ADP007952, female), 24 April 1911 (EA Schwarz) (NMNH: ADP007950, male), $2.2 \mathrm{~km}$ W Frijoles, $9.1736^{\circ} \mathrm{N}, 79.7966^{\circ} \mathrm{W}, 37 \mathrm{~m}, 25$ July 1977 (RB Kimsey, LS Kimsey)(UCD: ADP058775, female), 4.8 km W Cocoli, $8.980^{\circ} \mathrm{N}$, $79.629^{\circ} \mathrm{W}, 188 \mathrm{~m}, 30$ August 1974, HP Stockwell, 046080, female), 8 km NW Gamboa, $9.1639^{\circ} \mathrm{N}, 79.7492^{\circ} \mathrm{W}, 100 \mathrm{~m}, 15$ July 1976 (TL Erwin, et al.)(NMNH: ADP055841, male), Barro Colorado Nature Monument, Barro Colorado Island, Barro Colorado Research Station, $9.1652^{\circ} \mathrm{N}, 79.8368^{\circ} \mathrm{W}, 70 \mathrm{~m}, 26$ June 1926 (NMNH: ADP 007951, female), May 1929 (PJ Darlington Jr.)(MCZ: ADP007948, male, 007957, female), 2 July 1938 (EC Williams)(CMNH: ADP007956, male), January 1941 (KW Cooper) (MCZ: ADP007949, ADP007955, females), April - May 1942 (NMNH: ADP007954, female), 19 February 1975 (TL Erwin, JL Lawrence)(NMNH: ADP027571, female), March 1975 (TL Erwin, JL Lawrence)(NMNH: ADP042850, male), 10 May 1977 (H Wolda)(NMNH: ADP079070, male), 2 June 1977 (H Wolda)(NMNH: ADP077804, female), 5 June 1977 (H Wolda)(NMNH: ADP078274, female), 25 September 1977 (H Wolda)(NMNH: ADP076934, male), 1 November 1977 (H Wolda)(NMNH: ADP082523, male), 24 April 1978 (H Wolda)(NMNH: ADP089657, male), 24 April 1978 (H Wolda)(NMNH: ADP089660, female), 19 May 1978 (H Wolda)(NMNH: ADP062493, male), 22 May 1978 (H Wolda)(NMNH: ADP066554, male), 13 June 1978 (H Wolda)(NMNH: ADP065729, female), 4 October 1978 (H Wolda)(NMNH: ADP080371, female), Parque Nacional Soberania, Madden Forest, $9.098^{\circ} \mathrm{N}, 79.616^{\circ} \mathrm{W}$, 50m, 3 July 1974 (C O'Brien, L O’Brien, B Marshall)(NMNH: ADP027330, male); Cocle, El Valle, $8.603^{\circ} \mathrm{N}, 80.152^{\circ} \mathrm{W}, 800 \mathrm{~m}, 16$ May 1973 (HP Stockwell)(NMNH: ADP047483, female), El Valle, $8.603^{\circ} \mathrm{N}, 80.152^{\circ} \mathrm{W}, 829 \mathrm{~m}, 26$ May 1983 (WE Steiner) (NMNH: ADP005802, female); Panamá, Parque Natural Metropolitano, Panamá City, 8.9946 N, 79.5428 W, 67m, 13 May 1996 (F. Øedegaard)(NMNH: ADP140497, male), 27 September 1995, (F. Øedegaard)(NMNH: ADP140507, male), Portobelo, $9.555^{\circ} \mathrm{N}, 69.653^{\circ} \mathrm{W}, 10 \mathrm{~m}, 11$ March 1911 (EA Schwarz)(NMNH: ADP007953, female). Perú, Loreto, Pacaya-Samiria National Reserve, Río Samiria, Cocha Shinguito, 5.1775 ${ }^{\circ} \mathrm{S}, 76.6556^{\circ} \mathrm{W}, 112 \mathrm{~m}, 19$ June 1990 (TL Erwin, et al.)(NMNH: ADP094065, female), 20 August 1991 (TL Erwin, MG Pogue)(NMNH: ADP051383, female), 22 May 1990 (TL Erwin, et al.)(NMNH: ADP086946, ADP086980, ADP086980, females); 
Madre de Dios, Reserva Nacional Tambopata, $30 \mathrm{~km}$ (air) SW, Puerto Maldonado, Explorer's Inn, $12.8364^{\circ} \mathrm{S}, 69.2936^{\circ} \mathrm{W}, 209 \mathrm{~m}, 25$ February 1984 (TL Erwin, et al.) (NMNH: ADP007594, female), Manu Reserved Zone, Río Manu, BIOLAT Biological Station, Pakitza, $11.9446^{\circ} \mathrm{S}, 71.2831^{\circ} \mathrm{W}, 356 \mathrm{~m}, 9$ September 1988 (TL Erwin)(NMNH: ADP007556, male), 14 October 1991 (TL Erwin, MG Pogue)(NMNH: ADP007555, male), 10 July 1992 (TL Erwin, GP Servat, D Silva, F Pfuno S, E Pfuno S)(NMNH: ADP007572, female), 22 June 1993 (TL Erwin, F Pfuno S)(NMNH: ADP007557, female). Suriname, Kwatta, Warwabos, weg nr zee, $5.8437^{\circ} \mathrm{N}, 55.1586^{\circ} \mathrm{W}, 6 \mathrm{~m}, 28 \mathrm{Janu}-$ ary - 1 February 1964 (DC Geijskes)(NBCL: ADP005808, male, ADP005806, female); Paramaribo, Ma Retraite, $5.8437^{\circ} \mathrm{N}, 55.1586^{\circ} \mathrm{W}, 6 \mathrm{~m}, 12-14$ February 1964 (DC Geijskes)(NBCL: ADP005807, male), WANICA, Lelydorp, Sumatra weg, $5.700^{\circ} \mathrm{N}, 55.198^{\circ} \mathrm{W}$, 7m, 26-31 March 1964 (DC Geijskes)(NBCL: ADP007598, female). Trinidad and ToBago, Tunapuna, Mt. St. Benedict Monastery, nr. PAX House, $10.6624^{\circ} \mathrm{N}, 61.3984^{\circ} \mathrm{W}$, 204m, 8-9 July 1999 (GB Edwards)(NMNH: ADP112207, male, ADP112233, female). Venezuela, Bolivar, $22 \mathrm{~km}$ E Upata, $7.960^{\circ} \mathrm{N}, 62.212^{\circ} \mathrm{W}, 359 \mathrm{~m}, 18-19$ June 1996 (HF Howden, A Howden)(NMNH: ADP007596, female).

Geographic distribution (Fig. 11). This species is currently known from the restricted type locality (see above) in Colombia, and from Brazil - Amazonas; Ecuador, French Guiana, Panamá, Perú, Suriname, Trinidad and Tobago, and Venezuela.

Notes. Reichardt (1973) reported additional localities under his treatment for this name; however, he also synonymized $H$. verrucosa with $H$. tuberculata in Reichardt (1971). Since we do not accept his action and regard them both as good species, we cannot use his locality records without access to the specimens he saw in order for us to correct his identifications.

\section{Summary and future directions}

Most of the 738 specimens used in this study were taken from the rainforest canopy, or upper understory, using insecticidal fogging techniques. One $H$. auxilidora adult was found by George Vogt in Texas under the webbing of a live colony of Psocoptera, reminiscent of adults of Hyboteroides Erwin \& Ball species that live in the colonies of Embioptera (Embiidina) under their webbing (Erwin and Ball 2012). These rather flattened "blattiform" beetles with dorso-ventrally flattened legs and depressed bodies may live normally under the silken nets of their hosts on tree trunks and branches in the canopy and understory. If so, they may prey on the hosts with their long stiletto-like galea apices and lacinial teeth and numerous long setae on the mouthparts (reminding one of the Collembola-seizing adults of Loricera and Leistus). Such mouthparts may aid in capturing soft-bodied psocids and embiids. Adults of Hyboptera are also known from large bombacaceous anther rings on the forest floor in the dry season (Barro Colorado Island, Panamá - Pseudobombax septenatum (Jacq.) Dugand) and from FITs, malaise traps, SLAM traps, and UV/White lights. Amongst the lineages of Cryptobatida, both Hybopteroides and Thoasia adults share several structural attributes, in addition to the 
mouthparts mentioned above, with Hyboptera adults, such as a serial row of long setae on three or more elytral intervals, angulate (or, subangulate) lateral margin of the pronotum, short antennae, broadly depressed mandibles, etc.

With regard to the genus Hyboptera, the recent discoveries of several new species in remote parts of the upper Amazon Basin suggests that further sampling in such areas will increase the species richness of this markedly (structurally and behaviorally) interesting (architecturally and behaviorally) lineage of Carabidae. We also note that even though at present there are not many species known and adults are morphologically markedly modified from more "typical" carabids, and have a unique way of life preying on insects under webbing, the lineage is widely dispersed from Texas to southeastern Brazil with many species that are widespread in their distributions.

Adults of the (currently) monobasic Thoasia Liebke, 1939 are exceedingly common in canopy fogging samples (Erwin 1991); however, nothing is known about their way of life and they are only known with precise location from foggings in Perú and Ecuador and FIT samples in French Guiana. Feeding specializations such as those hypothesized herein for adult Hyboptera and Hybopteroides and commonality of morphological attributes offer a fertile field of study on Thoasia for coleopterists eager to spend long periods of time in the rainforest canopies. However, before that, Thoasia is in need of a taxonomic revision and three undescribed species need to be treated (cf. Erwin et al. 2012; Erwin, in prep). Liebke's holotype of the type species, Thoasia rugifrons, is in the Polish Academy of Sciences Collection according to Mroczkowski (1960).

\section{Acknowledgements}

In addition to those persons mentioned under Methods and Materials above, we extend hearty thanks to Charyn Micheli and Karolyn Darrow (both of the Department of Entomology at the Smithsonian Institution) for friendly review of the manuscript and data provided in Appendix 1(CM), and adult habitus and map images, review of the Figure Captions, as well as illustrations of the male and female genitalia (KD). We also warmly thank Charlotte Aldebron (Department of Entomology at the Smithsonian Institution) for assistance with dissections of male and female genitalia.

\section{References}

Ball GE (1972) Classification of the species of the Harpalus subgenus Glanodes Casey (Carabidae, Coleoptera). The Coleopterists Bulletin 26: 179-204.

Chaudoir M de (1872) Monographie des callidides. Annales de la Sociéte' Entomologique de Belgique 15: 97-204.

Chaudoir M de (1877) Genres nouveaux et espèces inédites de la familla des carabiques. Bulletin de la Société Impériale des naturalistas de Moscou 52: 188-268. 
Dejean PFMA (1825) Species général des coléoptères, de la collection de M. le Comte Dejean. Crevot, Paris, 463 pp.

Dejean PFMA (1831) Species général des coléoptères, de la collection de M. le Comte Dejean, 5. Méquignon-Marvis, Paris, 883 pp.

Erwin TL (1974) Studies of the subtribe Tachyina (Coleoptera: Carabidae: Bembidiini), Part II: a revision of the New World Australian genus Pericompsus LeConte. Smithsonian Contributions to Zoology 162: 1-96. https://doi.org/10.5479/si.00810282.162

Erwin TL (1991) Natural history of the carabid beetles at the BIOLAT Rio Manu Biological Station, Pakitza, Perú. Revista Peruana de Entomologia (1990)33: 1-85.

Erwin TL (2004) The Beetle Family Carabidae of Costa Rica and Panamá: Descriptions of four new genera and six new species with notes on their way of life (Insecta: Coleoptera). Zootaxa 537: 1-18. https://doi.org/10.11646/zootaxa.537.1.1

Erwin TL (2011a) A Treatise on the Western Hemisphere Caraboidea (Coleoptera): Their classification, distributions, and ways of life Volume III (Carabidae - loxomeriformes, melaeniformes). Pensoft, Sofia-Moscow, 412 pp.

Erwin TL (2011b) Halocoryza Alluaud 1919, sea-side beetles of the Atlantic, Pacific, and Indian Oceans: a generic synopsis and description of a remarkable new species from Baja California (Coleoptera, Carabidae, Scaritini, Clivinina). ZooKeys 127: 1-13. https://doi. org/10.3897/zookeys.127.1748

Erwin TL, Ball GE (2011) Badister Clairville 1806 (Coleoptera: Carabidae: Licinini): A new species and new continental record for the genus in Amazonian Perú. In: Erwin TL (Ed.) Proceedings of a symposium honoring the careers of Ross and Joyce Bell and their contributions to scientific work. Burlington, Vermont, 12-15 June 2010. ZooKeys 147: 399-417.

Erwin TL, Ball GE (2012) Hybopteroides, a new genus in the Cryptobatida group of subtribe Agrina, with three new species and notes on their way of life (Insecta: Coleoptera, Carabidae, Lebiini). Pan-Pacific Entomologist 88(2): 188-201. https://doi.org/10.3956/201229.1

Erwin TL, Erwin LJM (1976) Relationships of predaceous beetles to tropical forest wood decay. Part II. The natural history of Neotropical Eurycoleus macularis Chevrolat (Carabidae: Lebiini) and its implications in the evolution of ectoparasitoidism. Biotropica 8(4): 215-224. https://doi.org/10.2307/2989713

Erwin TL, Johnson PJ (2000) Naming species, a new paradigm for crisis management in taxonomy: rapid journal validation of scientific names enhanced with more complete descriptions on the internet. The Coleopterists Bulletin 54(3): 269-278. https://doi. org/10.1649/0010-065X(2000)054[0269:NSANPF]2.0.CO;2

Erwin TL, Kavanaugh DH (1981) Systematics and zoogeography of Bembidion Latreille: I. The carlhi and erasum groups of western North America (Coleoptera: Carabidae, Bembidiini). Entomologica Scandinavica Supplement 15: 33-72.

Erwin TL, Zamorano LS (2014) A synopsis of the Tribe Lachnophorini, with a new genus of Neotropical distribution and a revision of the Neotropical genus Asklepia Liebke 1938 (Insecta: Coleoptera: Carabidae). ZooKeys 430: 1-108. https://doi.org/10.3897/zookeys.430.8094 
Eschscholtz JF (1829) Zoologischer Atlas, enthalend Abbildungen und Beschreibungen neuer Theirarten, während des Flottvapitains von Kotzebue zweiter Reise um die Welt, auf der Russiisch-Kaiserlichen Kriegsschlupp Predpriaetië in den Jahren 1823-1826, Erstes Heft. Reimer, Berlin, 17 pp.

Kavanaugh DH (1979) Studies on the Nebriini (Coleoptera: Carabidae), III. New Nearctic Nebria species and subspecies, nomenclatural notes, and lectotype designations. Proceedings of the California Academy of Sciences 42: 87-133.

Kavanaugh DH, Erwin TL (1991) The Tribe Cicindini Bänninger (Coleoptera: Carabidae): Comparative morphology, natural history, and reclassification. Proceedings of the Entomological Society of Washington 93(2): 356-389.

Liebke M (1939) Neue Laufkäfer. Festschrift zum 60. Geburtstage von Profesor Dr. Embrik Strand. 5: 91-130.

Liu Y, Kavanaugh DH, Shi HL, Liang HB (2011) A Key to species of subgenus Lithochlaenius (Coleoptera, Carabidae, Chlaeniini, Chlaenius), with descriptions of three new species. ZooKeys 128: 15-52. https://doi.org/10.3897/zookeys.128.1804

Lorenz W (1998) Systematic list of extant ground beetles of the world (Insecta, Coleoptera "Geadephaga": Trachypachidae and Carabidae incl. Paussinae, Cicinidelinae, Rhysodinae). Privately published, W. Lorenz, Tutzing, 502 pp.

Lorenz W (2005) Systematic list of extant ground beetles of the world (Insecta, Coleoptera "Geadephaga": Trachypachidae and Carabidae incl. Paussinae, Cicinidelinae, Rhysodinae). 2nd Edition, Privately published, W. Lorenz, Tutzing, 530 pp.

Mateu J (1961) Sexta nota sobre los Lebiidae neotropicales (Coleópteros - Carábidos). Estratto dagli annali del Museo Civico di Storia Naturale di Genova 72: 161-178.

Mroczkowski M (1960) List of type specimens in the collection of the Institute of Zoology of the Polish Academy of Sciences in Warszawa III. Carabidae (Coleoptera). Annales Zoologici 18(21): 365-409.

Oberthür R (1884) Liste des Carabiques récoltés á Saint-Laurent-du-Maroni en 1878 et 1879 par M. le Dr. Charles Nodier médecin de la Marine et description desespèces nouvelles. Coleopterorum Novitates 1: 51-54.

Putzeys JAAH (1845) Prémices entomologiques. Mémoires de la Société Royale des Sciences de Liège, (1845-1846) 2: 353-417.

Reichardt H (1971) Carabidae (Coleoptera) neotropicais: Espécies Venezuelanas e formas relcionadas. Papéis Avulsos de Zoología 24(5): 73-91.

Reichardt H (1973) A review of Hyboptera Chaoudoir (Coleoptera, Carabidae). Revista Brasileira de Entomologia 17(8): 47-55.

Reiche LJ (1842) Coléoptèra de Colombie. Revue Zoologique. 1842: 238-242, 272-276, 307-314, 374-378.

Shpeley D, Ball GE (2001) A taxonomic review of the subtribe Pericalina (Carabidae: Lebiini) in the Western Hemisphere, with descriptions of new species and notes about classification and zoogeography. Insecta Mundi (2000) 14: 1-185.

Snodgrass RE (1935) Principles of Insect Morphology. McGraw-Hill Book Company, Inc, New York, $667 \mathrm{pp}$. 


\section{Appendix I}

Morphological measurements and ratios for adults of species of Hyboptera Chaudoir 1872. All values are in millimeters. Apparent body length (ABL) is also provided in the descriptions. Means provided for ratios are "harmonic means."

\section{angulicollis specigroup}

A. Hyboptera angulicollis Chaudoir

\begin{tabular}{l|c|c|c|c}
\hline \multirow{2}{*}{} & \multicolumn{2}{|c|}{ Males (N19) } & \multicolumn{2}{c}{ Females (11) } \\
\cline { 2 - 5 } & Range & Mean & Range & Mean \\
\hline Total Length (SBL) & $3.344-6.958$ & 3.695 & $3.353-4.148$ & 3.724 \\
\hline Maximum Width & $1.944-2.284$ & 2.135 & $1.924-2.532$ & 2.204 \\
\hline Width of Head / Width of Left Elytron & $0.836-0.977$ & 0.914 & $0.814-0.963$ & 0.891 \\
\hline Pronotum: Width (at widest part) / Length & $1.647-1.978$ & 1.81 & $1.718-1.909$ & 1.802 \\
\hline Length of Pronotum / Length of Head & $1.256-1.495$ & 1.364 & $1.279-1.515$ & 1.381 \\
\hline ABL & $3.943-4.800$ & 4.296 & $4.015-4.795$ & 4.377 \\
\hline
\end{tabular}

B. Hyboptera apollonia Erwin

\begin{tabular}{l|c|c|c|c}
\hline \multirow{2}{*}{} & \multicolumn{2}{|c}{ Males (N2) } & \multicolumn{2}{c}{ Females (N6) } \\
\cline { 2 - 5 } & Range & Mean & Range & Mean \\
\hline Total Length (SBL) & $3.723-4.263$ & 3.975 & $3.534-4.123$ & 3.835 \\
\hline Maximum Width & $2.168-2.502$ & 2.327 & $2.098-2.574$ & 2.3 \\
\hline Width of Head / Width of Left Elytron & $0.889-0.927$ & 0.908 & $0.810-0.914$ & 0.888 \\
\hline Pronotum: Width (at widest part) / Length & $1.703-1.714$ & 1.709 & $1.799-1.955$ & 1.861 \\
\hline Length of Pronotum / Length of Head & $1.230-1.353$ & 1.288 & $1.211-1.407$ & 1.32 \\
\hline ABL & $4.534-5.144$ & 4.82 & $4.199-5.067$ & 4.566 \\
\hline
\end{tabular}

C. Hyboptera biolat Erwin \& Henry, sp. n.

\begin{tabular}{l|c|c|c|c}
\hline \multirow{2}{*}{} & \multicolumn{2}{|c|}{ Males (N9) } & \multicolumn{2}{c}{ Females (N2) } \\
\cline { 2 - 5 } & Range & Mean & Range & Mean \\
\hline Total Length (SBL) & $3.159-3.744$ & 3.458 & $3.207-3.482$ & 3.339 \\
\hline Maximum Width & $1.728-2.328$ & 1.981 & $1.676-1.996$ & 1.822 \\
\hline Width of Head / Width of Left Elytron & $0.832-0.996$ & 0.937 & $0.964-1.058$ & 1.009 \\
\hline Pronotum: Width (at widest part) / Length & $1.738-1.965$ & 1.85 & $1.793-1.943$ & 1.865 \\
\hline Length of Pronotum / Length of Head & $1.300-1.429$ & 1.345 & $1.396-1.518$ & 1.455 \\
\hline ABL & $3.714-4.166$ & 3.969 & $3.638-3.855$ & 3.743 \\
\hline
\end{tabular}

D. Hyboptera vestiverdis Henry \& Erwin, sp. n.

\begin{tabular}{l|c|c|c|c}
\hline \multirow{2}{*}{} & \multicolumn{2}{|c|}{ Males (N15) } & \multicolumn{2}{c}{ Females (N15) } \\
\cline { 2 - 5 } & Range & Mean & Range & Mean \\
\hline Total Length (SBL) & $3.339-3.992$ & 3.611 & $3.135-3.956$ & 3.65 \\
\hline Maximum Width & $1.896-2.548$ & 2.115 & $1.826-2.428$ & 2.127 \\
\hline Width of Head / Width of Left Elytron & $0.834-1.016$ & 0.93 & $0.845-0.983$ & 0.924 \\
\hline Pronotum: Width (at widest part) / Length & $1.698-2.003$ & 1.861 & $1.680-1.993$ & 1.82 \\
\hline Length of Pronotum / Length of Head & $1.225-1.533$ & 1.354 & $1.284-1.519$ & 1.389 \\
\hline ABL & $3.958-4.623$ & 4.272 & $3.461-4.550$ & 4.201 \\
\hline
\end{tabular}


E. Hyboptera scheelea Erwin \& Henry, sp. n.

\begin{tabular}{l|c|c|c|c}
\hline \multirow{2}{*}{} & \multicolumn{2}{|c|}{ Males (N0) } & \multicolumn{2}{c}{ Females (N1) } \\
\cline { 2 - 5 } & Range & Mean & Range & Mean \\
\hline Total Length (SBL) & & & & 3.683 \\
\hline Maximum Width & & & & 2.1 \\
\hline Width of Head / Width of Left Elytron & & & & 0.956 \\
\hline Pronotum: Width (at widest part) / Length & & & & 1.921 \\
\hline Length of Pronotum / Length of Head & & & & 1.306 \\
\hline ABL & & & & 4.544 \\
\hline
\end{tabular}

F. Hyboptera shasta Erwin, sp. n.

\begin{tabular}{l|c|c|c|c}
\hline \multirow{2}{*}{} & \multicolumn{2}{|c|}{ Males (N1) } & \multicolumn{2}{c}{ Females (N0) } \\
\cline { 2 - 5 } & Range & Mean & Range & Mean \\
\hline Total Length (SBL) & & 3.885 & & \\
\hline Maximum Width & & 2.174 & & \\
\hline Width of Head / Width of Left Elytron & & 0.966 & & \\
\hline Pronotum: Width (at widest part) / Length & & 1.873 & & \\
\hline Length of Pronotum / Length of Head & & 1.361 & & \\
\hline ABL & & 4.819 & & \\
\hline
\end{tabular}

G. Hyboptera tepui Erwin \& Henry, sp. n.

\begin{tabular}{l|c|c|c|c}
\hline \multirow{2}{*}{} & \multicolumn{2}{|c|}{ Males (N0) } & \multicolumn{2}{c}{ Females (N1) } \\
\cline { 2 - 5 } & Range & Mean & Range & Mean \\
\hline Total Length (SBL) & & & & 4.289 \\
\hline Maximum Width & & & & 2.458 \\
\hline Width of Head / Width of Left Elytron & & & & 0.878 \\
\hline Pronotum: Width (at widest part) / Length & & & & 1.741 \\
\hline Length of Pronotum / Length of Head & & & & 1.309 \\
\hline ABL & & & & 5.155 \\
\hline
\end{tabular}

H. Hyboptera tiputini Erwin \& Henry, sp. n.

\begin{tabular}{l|c|c|c|c}
\hline \multirow{2}{*}{} & \multicolumn{2}{|c|}{ Males (N15) } & \multicolumn{2}{c}{ Females (N15) } \\
\cline { 2 - 5 } & Range & Mean & Range & Mean \\
\hline Total Length (SBL) & $3.331-4.421$ & 4.017 & $3.511-4.724$ & 4.166 \\
\hline Maximum Width & $2.008-2.924$ & 2.363 & $1.868-2.902$ & 2.432 \\
\hline Width of Head / Width of Left Elytron & $0.796-0.987$ & 0.897 & $0.819-1.017$ & 0.899 \\
\hline Pronotum: Width (at widest part) / Length & $1.699-2.076$ & 1.823 & $1.688-1.936$ & 1.812 \\
\hline Length of Pronotum / Length of Head & $1.293-1.517$ & 1.373 & $1.299-1.476$ & 1.367 \\
\hline ABL & $4.241-5.301$ & 4.819 & $4.211-5.640$ & 4.965 \\
\hline
\end{tabular}

I. Hyboptera viridivittis Chaudoir

\begin{tabular}{l|c|c|c|c}
\hline \multirow{2}{*}{} & \multicolumn{2}{|c|}{ Males (N3) } & \multicolumn{2}{c}{ Females (N5) } \\
\cline { 2 - 5 } & Range & Mean & Range & Mean \\
\hline Total Length (SBL) & $3.785-4.052$ & 3.933 & $3.759-4.167$ & 4.003 \\
\hline Maximum Width & $2.34-2.458$ & 2.399 & $2.184-2.586$ & 2.374 \\
\hline Width of Head / Width of Left Elytron & $0.841-0.892$ & 0.871 & $0.842-0.922$ & 0.893 \\
\hline Pronotum: Width (at widest part) / Length & $1.729-1.853$ & 1.798 & $1.765-1.930$ & 1.84 \\
\hline Length of Pronotum / Length of Head & $1.262-1.517$ & 1.355 & $1.199-1.316$ & 1.278 \\
\hline ABL & $4.787-5.089$ & 4.934 & $4.646-5.272$ & 5.003 \\
\hline
\end{tabular}




\section{tuberculata species group}

J. Hyboptera auxilidora Erwin

\begin{tabular}{l|c|c|c|c}
\hline \multirow{2}{*}{} & \multicolumn{2}{|c|}{ Males (N6) } & \multicolumn{2}{c}{ Females (N6) } \\
\cline { 2 - 5 } & Range & Mean & Range & Mean \\
\hline Total Length (SBL) & $4.389-4.938$ & 4.654 & $4.408-4.983$ & 4.755 \\
\hline Maximum Width & $2.42-3.28$ & 2.77 & $2.524-2.922$ & 2.726 \\
\hline Width of Head / Width of Left Elytron & $0.727-0.980$ & 0.88 & $0.875-0.936$ & 0.906 \\
\hline Pronotum: Width (at widest part) / Length & $1.774-1.988$ & 1.881 & $1.743-2.019$ & 1.868 \\
\hline Length of Pronotum / Length of Head & $1.208-1.297$ & 1.252 & $1.243-1.573$ & 1.361 \\
\hline ABL & $5.058-5.923$ & 5.483 & $5.265-5.925$ & 5.539 \\
\hline
\end{tabular}

K. Hyboptera dilutior Oberthür

\begin{tabular}{l|c|c|c|c}
\hline \multirow{2}{*}{} & \multicolumn{2}{|c|}{ Males (N15) } & \multicolumn{2}{c}{ Females (N15) } \\
\cline { 2 - 5 } & Range & Mean & Range & Mean \\
\hline Total Length (SBL) & $3.545-4.665$ & 4.256 & $3.888-4.559$ & 4.339 \\
\hline Maximum Width & $1.952-2.772$ & 2.416 & $2.708-3.208$ & 3.024 \\
\hline Width of Head / Width of Left Elytron & $0.909-1.012$ & 0.959 & $0.743-0.850$ & 0.786 \\
\hline Pronotum: Width (at widest part) / Length & $1.709-1.925$ & 1.811 & $1.701-2.003$ & 1.827 \\
\hline Length of Pronotum / Length of Head & $1.236-1.403$ & 1.333 & $1.210-1.350$ & 1.292 \\
\hline ABL & $3.916-5.979$ & 5.035 & $4.760-5.772$ & 5.129 \\
\hline
\end{tabular}

L. Hyboptera lucida Henry \& Erwin, sp. n.

\begin{tabular}{l|c|c|c|c}
\hline \multirow{2}{*}{} & \multicolumn{2}{|c|}{ Males (N5) } & \multicolumn{2}{c}{ Females (N9) } \\
\cline { 2 - 5 } & Range & Mean & Range & Mean \\
\hline Total Length (SBL) & $4.376-5.066$ & 4.752 & $4.690-5.265$ & 5.029 \\
\hline Maximum Width & $2.362-3.218$ & 2.849 & $2.820-3.248$ & 3.054 \\
\hline Width of Head / Width of Left Elytron & $0.845-0.976$ & 0.887 & $0.828-0.957$ & 0.883 \\
\hline Pronotum: Width (at widest part) / Length & $1.713-1.924$ & 1.84 & $1.742-1.981$ & 1.88 \\
\hline Length of Pronotum / Length of Head & $1.130-1.274$ & 1.207 & $1.150-1.351$ & 1.266 \\
\hline ABL & $5.641-6.294$ & 5.852 & $5.514-6.667$ & 6.093 \\
\hline
\end{tabular}

M. Hyboptera tuberculata (Dejean)

\begin{tabular}{l|c|c|c|c}
\hline \multirow{2}{*}{} & \multicolumn{2}{|c|}{ Males (N15) } & \multicolumn{2}{c}{ Females (N15) } \\
\cline { 2 - 5 } & Range & Mean & Range & Mean \\
\hline Total Length (SBL) & $3.942-4.853$ & 4.471 & $4.196-4.843$ & 4.533 \\
\hline Maximum Width & $2.178-2.928$ & 2.609 & $2.206-3.116$ & 2.689 \\
\hline Width of Head / Width of Left Elytron & $0.819-1.058$ & 0.899 & $0.839-1.029$ & 0.893 \\
\hline Pronotum: Width (at widest part) / Length & $1.731-2.223$ & 1.876 & $1.726-1.993$ & 1.859 \\
\hline Length of Pronotum / Length of Head & $1.103-1.379$ & 1.264 & $1.181-1.354$ & 1.277 \\
\hline ABL & $4.321-5.883$ & 5.231 & $4.745-5.763$ & 5.325 \\
\hline
\end{tabular}

N. Hyboptera verrucosa (Reiche)

\begin{tabular}{l|c|c|c|c}
\hline \multirow{2}{*}{} & \multicolumn{2}{|c|}{ Males (N14) } & \multicolumn{2}{c}{ Females (N16) } \\
\cline { 2 - 5 } & Range & Mean & Range & Mean \\
\hline Total Length (SBL) & $3.824-4.587$ & 4.203 & $3.793-4.898$ & 4.417 \\
\hline Maximum Width & $2.060-2.636$ & 2.352 & $2.190-2.846$ & 2.478 \\
\hline Width of Head / Width of Left Elytron & $0.893-1.025$ & 0.954 & $0.862-1.024$ & 0.928 \\
\hline Pronotum: Width (at widest part) / Length & $1.774-1.997$ & 1.882 & $1.797-2.012$ & 1.865 \\
\hline Length of Pronotum / Length of Head & $1.186-1.359$ & 1.269 & $1.185-1.398$ & 1.268 \\
\hline ABL & $4.493-5.511$ & 4.932 & $4.474-5.660$ & 5.06 \\
\hline
\end{tabular}

